\title{
From Conformal Group to Symmetries of Hypergeometric Type Equations
}

\author{
Jan DEREZINSSKI ${ }^{\dagger}$ and Przemystaw MAJEWSKI ${ }^{\dagger}$ \\ $\dagger$ Department of Mathematical Methods in Physics, Faculty of Physics, University of Warsaw, \\ Pasteura 5, 02-093 Warszawa, Poland \\ E-mail: Jan.Derezinski@fuw.edu.pl \\ URL: http://fuw.edu.pl/ derezins/ \\ $¥$ Bureau of Air Defence and Anti-missile Defence Systems, PIT-RADWAR S.A., \\ Poligonowa 30, 04-025 Warszawa, Poland \\ E-mail: Przemyslaw.Majewski@fuw.edu.pl \\ URL: http://fuw.edu.pl/ pmaj/
}

Received February 24, 2016, in final form October 20, 2016; Published online November 05, 2016 http://dx.doi.org/10.3842/SIGMA.2016.108

\begin{abstract}
We show that properties of hypergeometric type equations become transparent if they are derived from appropriate 2nd order partial differential equations with constant coefficients. In particular, we deduce the symmetries of the hypergeometric and Gegenbauer equation from conformal symmetries of the 4- and 3-dimensional Laplace equation. We also derive the symmetries of the confluent and Hermite equation from the so-called Schrödinger symmetries of the heat equation in 2 and 1 dimension. Finally, we also describe how properties of the ${ }_{0} F_{1}$ equation follow from the Helmholtz equation in 2 dimensions.
\end{abstract}

Key words: Laplace equation; hypergeometric equation; confluent equation; Kummer's table; Lie algebra; conformal group

2010 Mathematics Subject Classification: 35J05; 33Cxx; 35B06

\section{Contents}

1 Introduction $\quad 3$

1.1 Hypergeometric type operators $\ldots \ldots \ldots \ldots \ldots \ldots$

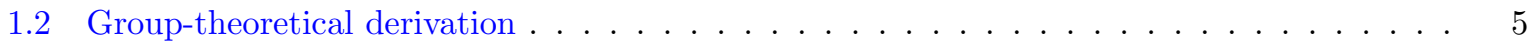

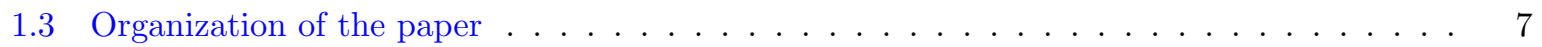

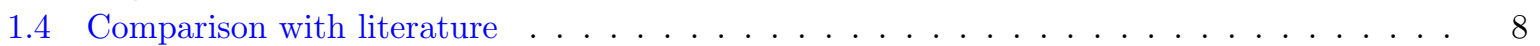

2 Hypergeometric type operators and their symmetries $\quad 9$

2.1 Hypergeometric operator . . . . . . . . . . . . . . . . . . . . 10

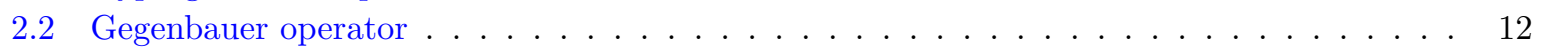

2.3 Confluent operator . . . . . . . . . . . . . . . . . . . . . . . 14

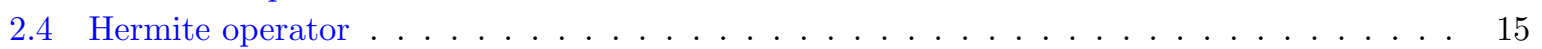

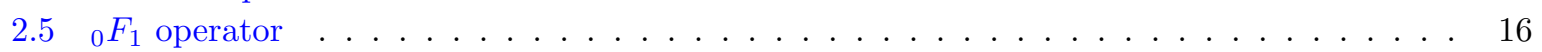

3 Basic complex geometry 16

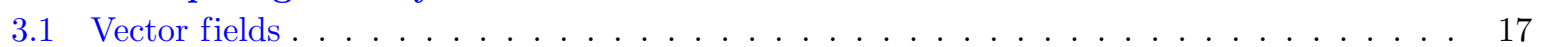

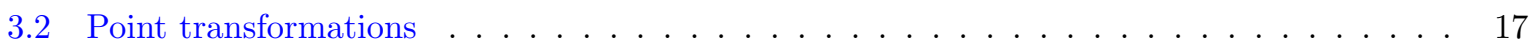

3.3 Local cocycles . . . . . . . . . . . . . . . . . . . . . . . . . . . . 18

3.4 Half-integer powers of a cocycle $\ldots \ldots \ldots \ldots \ldots \ldots \ldots$

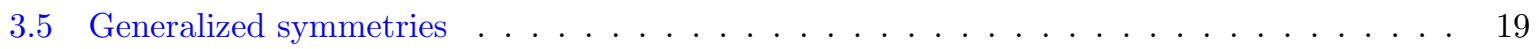


4 Line bundles $\quad 19$

4.1 Scaling . . . . . . . . . . . . . . . . . . . . . . . . . . 19

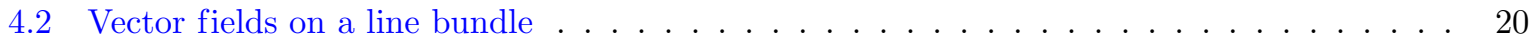

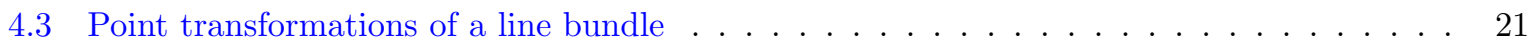

4.4 Homogeneous functions of integer degree . . . . . . . . . . . . . . . . . . . 21

4.5 Homogeneous functions of non-integer degree . . . . . . . . . . . . . . . . . 22

5 Complex Euclidean spaces $\quad 22$

5.1 Linear transformations . . . . . . . . . . . . . . . . . . . . . . . 22

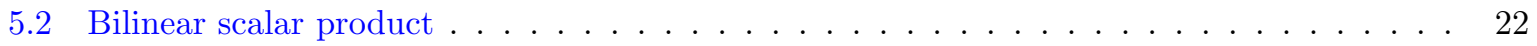

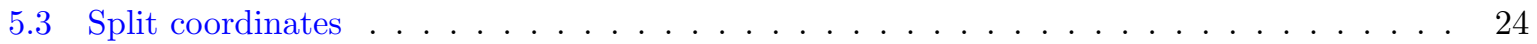

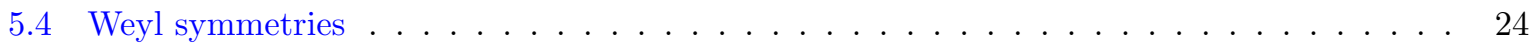

6 Conformal invariance $\quad 25$

6.1 Conformal invariance of Riemannian manifolds . . . . . . . . . . . . . . . . . . 25

6.2 Null quadric . . . . . . . . . . . . . . . . . . . . . . . . . . . 26

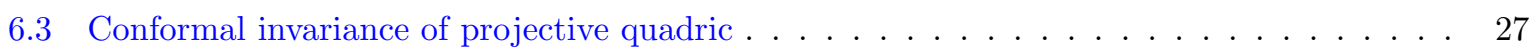

6.4 Conformal invariance of complex Euclidean space . . . . . . . . . . . . . . . . 28

6.5 Laplacian on bundle of homogeneous functions . . . . . . . . . . . . . . . . . 29

6.6 Conformal invariance of Laplacian for a general section . . . . . . . . . . . . . . . . . . 31

6.7 Conformal invariance of Laplacian on $\mathbb{C}^{n} \ldots \ldots \ldots \ldots \ldots$

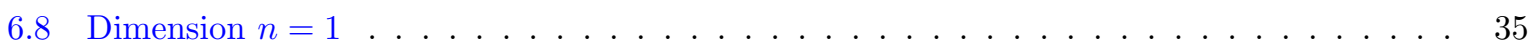

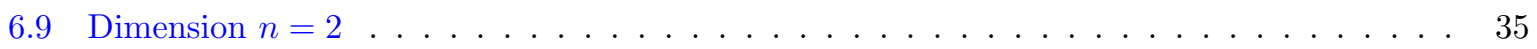

$\begin{array}{lll}7 & \mathrm{so}\left(\mathbb{C}^{6}\right) \text { and the hypergeometric equation } & \mathbf{3 6}\end{array}$

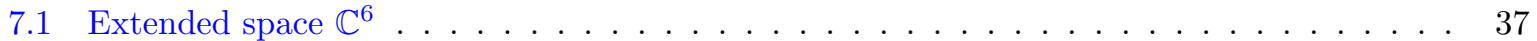

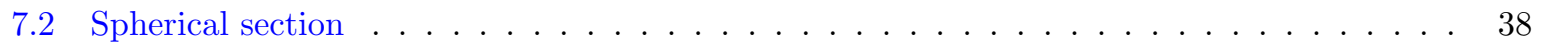

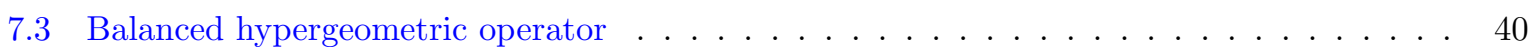

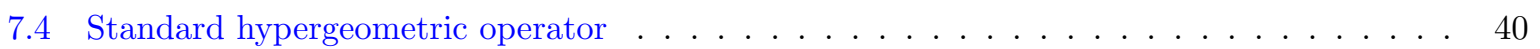

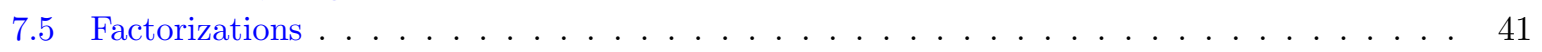

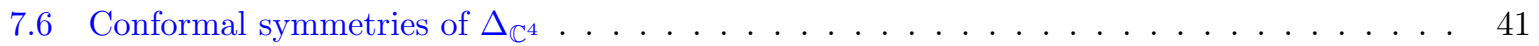

7.7 Deriving balanced hypergeometric operator from $\Delta_{\mathbb{C}^{4}} \ldots \ldots \ldots \ldots \ldots \ldots$

7.8 Deriving standard hypergeometric operator from $\Delta_{\mathbb{C}^{4}} \ldots \ldots \ldots \ldots \ldots \ldots$

8 so $\left(\mathbb{C}^{5}\right)$ and the Gegenbauer equation $\quad \mathbf{4 4}$

8.1 Extended space $\mathbb{C}^{5} \ldots \ldots \ldots \ldots \ldots \ldots \ldots \ldots \ldots$

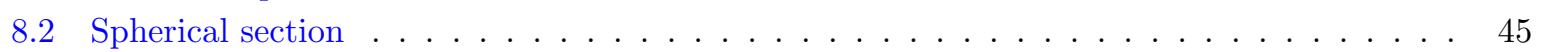

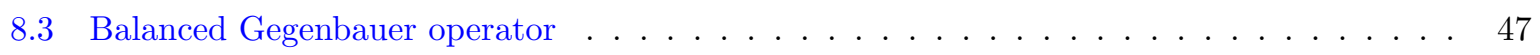

8.4 Standard Gegenbauer operator . . . . . . . . . . . . . . . . . . 47

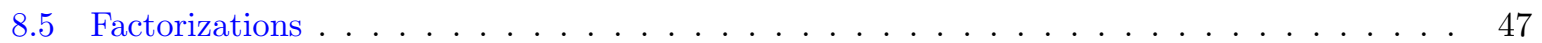

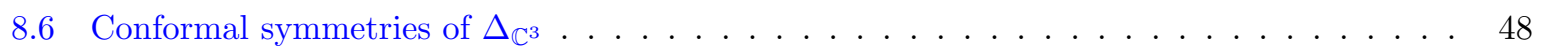

8.7 Deriving balanced Gegenbauer operator from $\Delta_{\mathbb{C}^{3}} \ldots \ldots \ldots \ldots \ldots \ldots$

8.8 Deriving standard Gegenbauer operator from $\Delta_{\mathbb{C}^{3}} \ldots \ldots \ldots \ldots \ldots$

9 Symmetries of the heat equation - the Schrödinger algebra $\quad 50$

9.1 The Schrödinger Lie algebra and group on $\mathbb{C}^{n+2} \ldots \ldots \ldots \ldots \ldots \ldots$

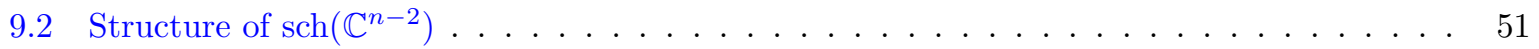

9.3 The Schrödinger Lie algebra and group on $\mathbb{C}^{n} \ldots \ldots \ldots \ldots \ldots$

9.4 The Schrödinger Lie algebra and group on $\mathbb{C}^{n-2} \oplus \mathbb{C} \ldots \ldots \ldots \ldots \ldots \ldots$

9.5 Hermite operator . . . . . . . . . . . . . . . . . . . . . . . . . . . . . . . . . . . . .

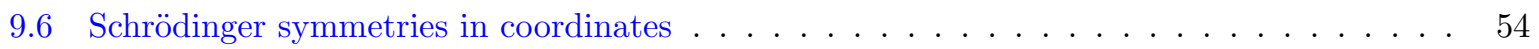


$10 \operatorname{sch}\left(\mathbb{C}^{2}\right)$ and the confluent equation $\quad \mathbf{5 7}$

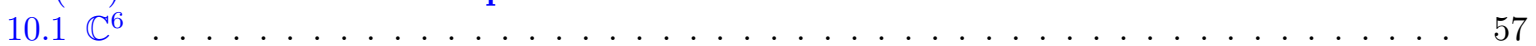

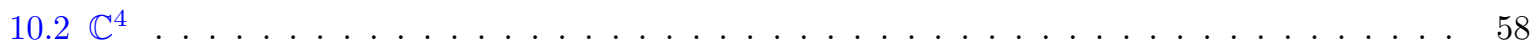

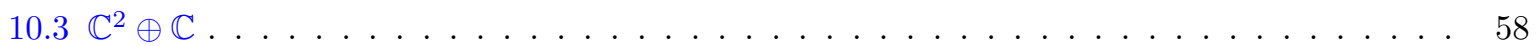

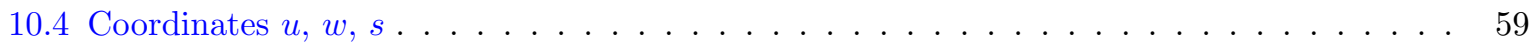

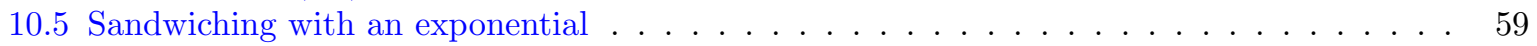

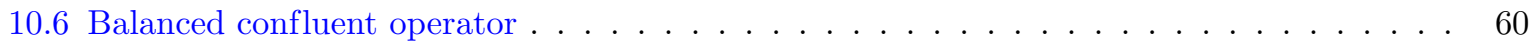

10.7 Standard confluent operator . . . . . . . . . . . . . . . . . . . . . 60

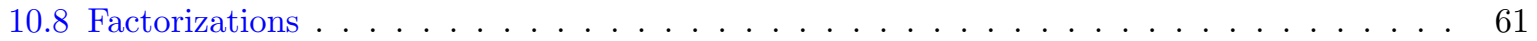

$11 \operatorname{sch}\left(\mathbb{C}^{1}\right)$ and the Hermite equation $\quad 61$

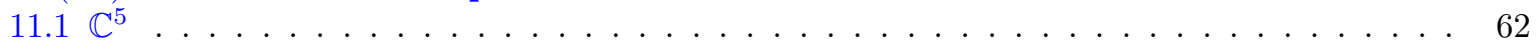

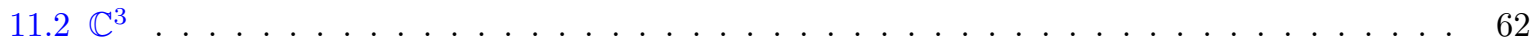

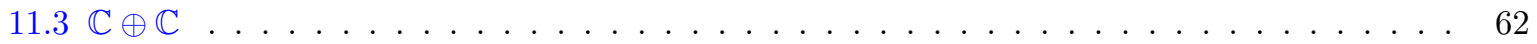

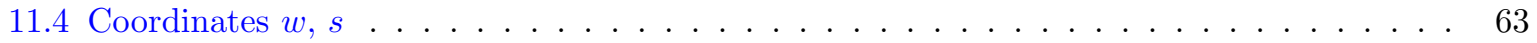

11.5 Sandwiching with a Gaussian . . . . . . . . . . . . . . . . . 63

11.6 Balanced Hermite operator _. . . . . . . . . . . . . . . . . . . . . 64

11.7 Standard Hermite operator _. . . . . . . . . . . . . . . . . . . . 64

11.8 Factorizations . . . . . . . . . . . . . . . . . . . . . 64

$12 \mathbb{C}^{2} \rtimes \mathrm{so}\left(\mathbb{C}^{2}\right)$ and the ${ }_{0} F_{1}$ equation $\quad \mathbf{6 5}$

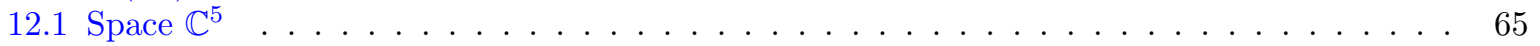

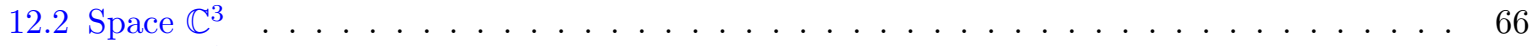

12.3 Space $\mathbb{C}^{2}$ and the Helmholtz equation $\ldots \ldots \ldots \ldots$

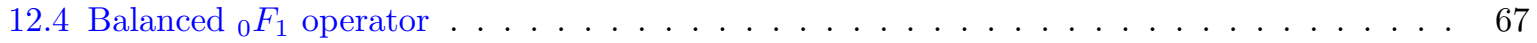

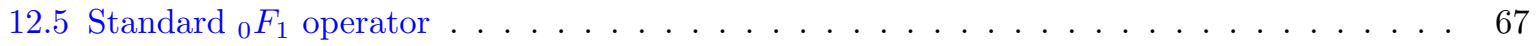

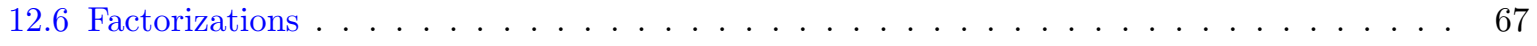

$\begin{array}{ll}\text { References } & 68\end{array}$

\section{Introduction}

The paper is devoted to the properties of the hypergeometric equation

$$
\left(z(1-z) \partial_{z}^{2}+(c-(a+b+1) z) \partial_{z}-a b\right) F(z)=0,
$$

the Gegenbauer equation

$$
\left(\left(1-z^{2}\right) \partial_{z}^{2}-(a+b+1) z \partial_{z}-a b\right) F(z)=0,
$$

the confluent equation

$$
\left(z \partial_{z}^{2}+(c-z) \partial_{z}-a\right) F(z)=0
$$

the Hermite equation

$$
\left(\partial_{z}^{2}-2 z \partial_{z}-2 a\right) F(z)=0
$$

and the ${ }_{0} F_{1}$ equation (closely related to the Bessel equation, see, e.g., [6])

$$
\left(z \partial_{z}^{2}+c \partial_{z}-1\right) F(z)=0 .
$$

Here, $z$ is a complex variable, $\partial_{z}$ is the differentiation with respect to $z$, and $a, b, c$ are arbitrary complex parameters.

Special functions that solve these equations are typical representatives of hypergeometric type functions [34]. They often appear in applications [11,32]. In old times they were considered one 
of the central topics of mathematics, see, e.g., [48]. In our opinion, they indeed belong to the most natural objects in mathematics.

Properties of hypergeometric type functions look quite complicated. According to our observations, when these properties are discussed, most people react with boredom and/or irritation. We would like to convince the reader that in reality identities related to hypergeometric type equations are beautiful and can be derived in an elegant and transparent way.

We will show that in order to understand hypergeometric type equations it is helpful to start from certain 2nd order PDE's in several variables with constant coefficients. If we start from rather obvious properties of these PDE's, reduce the number of variables and change the coordinates, we can observe how these properties become more complicated. At the end one obtains relatively complicated sets of identities for hypergeometric type equations.

\section{$1.1 \quad$ Hypergeometric type operators}

Equations (1.1)-(1.5) are determined by an operator of the form

$$
\mathcal{C}=\mathcal{C}\left(z, \partial_{z}\right):=\sigma(z) \partial_{z}^{2}+\tau(z) \partial_{z}+\eta
$$

In our paper we will concentrate on the study of the operator $\mathcal{C}$ itself, rather than on individual solutions $F$ of the equation

$$
\mathcal{C} F=0 \text {. }
$$

Note, however, that properties of $F$ 's can be to a large extent inferred from the properties of $\mathcal{C}$ itself.

According to the terminology used in [34], and then in [6, 7], equations (1.1)-(1.5) belong to the class of hypergeometric type equations. This class is defined by demanding that $\sigma$ is a polynomial of at most 2nd order, $\tau$ is a polynomial of at most 1st order and $\eta$ is a number. More precisely, (1.1)-(1.5) constitute standard forms of all nontrivial classes of hypergeometric type equations, as explained, e.g., in [6].

Equations (1.1)-(1.5) depend on a number of (complex) parameters. For instance, in the case of the hypergeometric equation these parameters are $a, b, c$. We will prefer to use different sets of parameters introduced in a systematic way in [6], which are more convenient to express the symmetries of these equations. In [6] these new parameters were called the Lie-algebraic parameters. Indeed, as we will describe, they are eigenvalues of the "Cartan algebra" of appropriate Lie algebras. For instance, for the hypergeometric equation the Lie-algebraic parameters, denoted $\alpha, \beta, \mu$, are the differences of the indices at the three singular points.

We will prefer not to use the operators $\mathcal{C}$ directly. As explained in [6], we can write

$$
\mathcal{C}\left(z, \partial_{z}\right)=\rho^{-1}(z) \partial_{z} \rho(z) \partial_{z}+\eta
$$

which defines (up to a multiplicative factor) a certain function $\rho(z)$ called the weight. Following [6], the operator

$$
\mathcal{C}^{\text {bal }}\left(z, \partial_{z}\right):=\rho(z)^{\frac{1}{2}} \mathcal{C}\left(z, \partial_{z}\right) \rho(z)^{-\frac{1}{2}},
$$

will be called the balanced form of $\mathcal{C}$. The study of the balanced form is obviously equivalent to that of $\mathcal{C}$, since both are related by a simple similarity transformation. The original operator $\mathcal{C}$ will be sometimes called the standard form of $\mathcal{C}$.

We will consider 3 classes of identities:

(1) discrete symmetries, 
(2) transmutation relations,

(3) factorizations.

Discrete symmetries involve a transformation of the independent variable, together with a change of the parameters. The family of the discrete symmetries of the hypergeometric equation is especially famous. In the literature it is sometimes known under the name of the Kummer's table $[1,21,22,25]$.

The balanced form is especially convenient for a presentation of discrete symmetries, because some of them simply reduce to the change of sign of parameters.

Transmutation relations say that $\mathcal{C}$ multiplied from the right by an appropriate 1 st order operator equals $\mathcal{C}$ for shifted parameters multiplied from the left by a similar 1st order operator. In quantum physics the corresponding 1st order operators are often called creation/annihilation operators.

Factorizations say that $\mathcal{C}$ can be written as a product of two 1st order operators, up to an additional term that does not contain the independent variable. It is easy to see that factorizations imply transmutation relations, as described, e.g., in [6]. Factorizations play an important role in quantum mechanics. They are often interpreted as the manifestation of supersymmetry [5]. In quantum mechanics discussion of these factorizations has a long history going back at least to $[14]$.

Discrete symmetries, transmutation relations and factorizations are far from being trivial. Nevertheless, in our opinion they belong to the most elementary properties of hypergeometric type equations and functions. There exist many other properties, notably addition formulas and integral representations, which we view as more advanced. We do not consider them in our paper.

\subsection{Group-theoretical derivation}

We will see that all hypergeometric type equations can be obtained by separating the variables of 2nd order PDE's with constant coefficients. We will always use the complex variable, to avoid discussing various signatures of these PDE's.

Every such a PDE has a Lie algebra and a Lie group of generalized symmetries. In this Lie algebra we can fix a certain maximal commutative algebra, which we will call the "Cartan algebra". Operators whose adjoint action is diagonal in the "Cartan algebra" will be called "root operators". In the Lie group of generalized symmetries we will distinguish a discrete group, which we will call the group of "Weyl symmetries". This group will implement automorphisms of the Lie algebra leaving invariant the "Cartan algebra".

Note that in some cases the Lie algebra of generalized symmetries is semisimple, and then the names Cartan algebra, root operators and Weyl symmetries correspond to the standard names. In other cases the Lie algebra is non-semisimple, and then the names are less standard - this is the reason for the quotation marks that we use.

Parameters of hypergeometric type equation can be interpreted as the eigenvalues of elements of the "Cartan algebra". In particular, the number of parameters of a given class of equations equals the dimension of the corresponding "Cartan algebra". Each transmutation relation is related to a "root operator". Finally, each discrete symmetry of a hypergeometric type operator corresponds to a "Weyl symmetry" of the Lie algebra.

We can distinguish 3 kinds of PDE's of the complex variable with constant coefficients:

(1) The Laplace equation on $\mathbb{C}^{n}$

$$
\Delta_{\mathbb{C}^{n}} f=0
$$

whose Lie algebra of generalized symmetries is $\operatorname{so}\left(\mathbb{C}^{n+2}\right)$. 
(2) The heat equation on $\mathbb{C}^{n-2} \oplus \mathbb{C}$,

$$
\left(\Delta_{\mathbb{C}^{n-2}}+\partial_{t}\right) f=0
$$

whose Lie algebra of generalized symmetries is $\operatorname{sch}\left(\mathbb{C}^{n-2}\right)$, the so-called (complex) Schrödinger Lie algebra.

(3) The Helmholtz equation on $\mathbb{C}^{n-1}$,

$$
\left(\Delta_{\mathbb{C}^{n-1}}-1\right) f=0
$$

whose Lie algebra of symmetries is $\mathbb{C}^{n-1} \rtimes \operatorname{so}\left(\mathbb{C}^{n-1}\right)$.

Separating the variables in these equations usually leads to differential equations with many variables. Only in a few cases it leads to ordinary differential equations, which turn out to be of hypergeometric type. All these cases are described in the following table:

Table 1.

\begin{tabular}{ccccc}
\hline PDE & $\begin{array}{c}\text { Lie } \\
\text { algebra }\end{array}$ & $\begin{array}{c}\text { dimension of } \\
\text { Cartan algebra }\end{array}$ & $\begin{array}{c}\text { discrete } \\
\text { symmetries }\end{array}$ & equation \\
\hline$\Delta_{\mathbb{C}^{4}}$ & $\operatorname{so}\left(\mathbb{C}^{6}\right)$ & 3 & cube & hypergeometric \\
$\Delta_{\mathbb{C}^{3}}$ & $\operatorname{so}\left(\mathbb{C}^{5}\right)$ & 2 & square & Gegenbauer \\
$\Delta_{\mathbb{C}^{2}}+\partial_{t}$ & $\operatorname{sch}\left(\mathbb{C}^{2}\right)$ & 2 & $\mathbb{Z}_{2} \times \mathbb{Z}_{2}$ & confluent \\
$\Delta_{\mathbb{C}}+\partial_{t}$ & $\operatorname{sch}\left(\mathbb{C}^{1}\right)$ & 1 & $\mathbb{Z}_{4}$ & Hermite \\
$\Delta_{\mathbb{C}^{2}}-1$ & $\mathbb{C}^{2} \rtimes \mathrm{so}\left(\mathbb{C}^{2}\right)$ & 1 & $\mathbb{Z}_{2}$ & ${ }_{0} F_{1}$ \\
\hline
\end{tabular}

The Laplace equation on $\mathbb{C}^{n}$, the heat equation on $\mathbb{C}^{n-2}$ and the Helmholtz equation on $\mathbb{C}^{n-1}$ together with their generalized symmetries can be elegantly derived by an appropriate reduction from the Laplace equation in $n+2$ dimensions

$$
\Delta_{\mathbb{C}^{n+2}} K=0 .
$$

Thus, as follows from Table 1, to derive symmetries of the hypergeometric and confluent equations one should start from

$$
\Delta_{\mathbb{C}^{6}} K=0 .
$$

To derive the Gegenbauer, Hermite and ${ }_{0} F_{1}$ equation together with all its symmetries it is enough to start with

$$
\Delta_{\mathbb{C}^{5}} K=0 .
$$

It is easy to reduce the Laplace equation from 6 to 5 dimensions. Thus the Laplace equation in 6 dimensions is the "mother" of all hypergeometric type equations.

Let us describe these derivations in more detail.

- We start from (1.6), where the symmetries $\operatorname{so}\left(\mathbb{C}^{6}\right)$ are obvious. By what we call the conformal reduction, we can reduce $\Delta_{\mathbb{C}^{6}}$ to $\Delta_{\mathbb{C}^{4}}$, and then further to the hypergeometric operator. Alternatively, one can reduce $\Delta_{\mathbb{C}^{6}}$ to an appropriate Laplace-Beltrami operator, and then we obtain (1.1) more directly.

- We can repeat an analogous procedure one dimension lower. We start from (1.7), and at the end we obtain the Gegenbauer operator. 
- One can reduce (1.6) to $\Delta_{\mathbb{C}^{2}}+\partial_{t}$, the heat operator in 2 dimensions, and then further to the confluent operator. Note that $\operatorname{sch}\left(\mathbb{C}^{2}\right)$ is contained in $\operatorname{so}\left(\mathbb{C}^{6}\right)$.

- One can repeat the above steps one dimension lower, reducing (1.7) to $\Delta_{\mathbb{C}}+\partial_{t}$, the heat operator in 1 dimension, and then further to the Hermite operator. Note that $\operatorname{sch}(\mathbb{C})$ is contained in $\operatorname{so}\left(\mathbb{C}^{5}\right)$.

- To obtain the ${ }_{0} F_{1}$ operator one needs to separate variables in the Helmholtz operator $\Delta_{\mathbb{C}^{2}}-1$. Its symmetries $\mathbb{C}^{2} \rtimes \mathrm{so}\left(\mathbb{C}^{2}\right)$ are contained in $\mathrm{so}\left(\mathbb{C}^{5}\right)$ and one can start the derivation from (1.7).

One can ask whether Table 1 can be enlarged, e.g., by considering $\Delta_{\mathbb{C}^{n}} f=0$ with its conformal symmetries so $\left(\mathbb{C}^{n+2}\right)$ for $n \geq 5$. One can argue that the answer is negative and Table 1 is complete. Indeed, the Cartan algebra of so $(n+2)$ has dimension $[n / 2]$, and $n-[n / 2]>1$ for $n \geq 5$. Therefore, separation of variables in the Laplace equation in dimension $n \geq 5$ leads to a differential equation in more than one variable.

\subsection{Organization of the paper}

The paper can be considered as a sequel to [6]. Nevertheless, it is to a large degree self-contained and independent of [6].

In Section 2 we list the identities that we would like to derive/explain in our article. As described in the introduction, these identities involve 5 classes of differential operators (1.1)-(1.5). All these operators are first transformed to the balanced form.

The versions of these identities for the standard form of equations (1.1)-(1.5) can be found in [6]. In order to reduce the length of the paper, in this paper we concentrate on the balanced form, which is more symmetric.

Sections 3, 4 and 5 provide basic definitions and concepts, mostly related to (complex) differential geometry, Lie groups and Lie algebras. This material is very well known, especially in the real context. Unfortunately, the use of complex manifolds, natural in our context, has some disadvantages due to the rigidity of holomorphic functions and their multivaluedness. This is the reason for some annoying minor complications in these sections, such as local representations of groups.

In Section 6 we describe the action of the conformal group/Lie algebra in $n$ dimensions. We do this first for a general $n$. As a simple, but instructive exercise we consider the cases $n=1,2$.

In Section 7 we consider the case $n=4$, which yields the hypergeometric operator. In Section 8 we consider $n=3$, which leads to the Gegenbauer operator. These two sections are very parallel to one another. Both are direct applications of the formalism of Section 6 .

In Section 9 we consider the Schrödinger group $\operatorname{Sch}\left(\mathbb{C}^{n-2}\right)$ and its Lie algebra $\operatorname{sch}\left(\mathbb{C}^{n-2}\right)$. They describe generalized symmetries of the heat equation in $n-2$ dimensions. We first do this for a general dimension.

In Section 10 we consider the case $n=4$, which yields the confluent operator. In Section 11 we consider $n=3$, which leads to the Hermite operator. Again, these two sections are quite parallel. They are applications of the formalism of Section 9.

In the final Section 12 we consider the Helmholtz equation in 2 dimensions together with the affine Euclidean symmetries $\mathbb{C}^{2} \rtimes \mathrm{O}\left(\mathbb{C}^{2}\right)$ and $\mathbb{C}^{2} \rtimes$ so $\left(\mathbb{C}^{2}\right)$. This leads to the ${ }_{0} F_{1}$ equation (or, equivalently, to the Bessel equation). We included this section for completeness, however its material is well-known and well documented in the literature.

Note that Sections 3, 4, 5, 6 and 9 are quite general and abstract. On the other hand, Sections 7, 8, 10, 11 and 12 are more concrete and present applications of the general theory to the classes of hypergeometric type equations (1.1), (1.2), (1.3), (1.4) and (1.5), respectively. To a large extent they can be read independently of the "general" part of the paper. 


\subsection{Comparison with literature}

Properties of functions of hypergeometric type are described in numerous books, such as $[1,8$, 9, 10, 13, 23, 26, 35, 38, 48]. In particular, the properties presented in Section 2 (transmutation relations, discrete symmetries and factorizations) are known in one form or another. A similar presentation can be found in [6]. (Unlike in this paper, the presentation of [6] involves the standard form of hypergeometric type equations and not their balanced form).

Lie algebras associated with the Bessel and Hermite functions can be found in papers by Weisner [46, 47].

The idea of studying hypergeometric type equations with help of Lie algebras was developed further by Miller. His early book [27] considers mostly small Lie algebras/Lie groups, typically $\mathrm{sl}(2, \mathbb{C}) / \mathrm{SL}(2, \mathbb{C})$ and its contractions, and applies them to obtain various identities about hypergeometric type functions. These Lie algebras have 1-dimensional "Cartan algebras" and a single pair of roots. This kind of analysis is able to explain only a single pair of transmutation relations, whereas to explain bigger families of transmutation relations one needs larger Lie algebras.

A Lie algebra strictly larger than $\operatorname{sl}(2, \mathbb{C})$ is $\operatorname{so}(4, \mathbb{C})$. There exists a large literature on the relation of the hypergeometric equation with so $(4, \mathbb{C})$ and its real forms, see, e.g., [16]. This Lie algebra is however still too small to account for all symmetries of the hypergeometric equation its Cartan algebra is only 2-dimensional, whereas the equation has three parameters.

An explanation of symmetries of the Gegenbauer equation in terms of so(5) and of the hypergeometric equation in terms of $\mathrm{so}(6) \simeq \operatorname{sl}(4)$ was first given by Miller, see [30], and especially [29].

Miller and Kalnins wrote a series of papers where they studied the symmetry approach to separation of variables for various 2 nd order partial differential equations, such as the Laplace and wave equation, see, e.g., [15]. A large part of this research is summed up in the book by Miller [31]. As an important consequence of this study, one obtains detailed information about symmetries of hypergeometric type equations.

The main tool that we use to describe properties of hypergeometric type functions is the theory of generalized symmetries of 2nd order linear PDE's. This theory is described in another book by Miller [28], and further developed in [31].

The fact that conformal transformations of the Euclidean space are generalized symmetries of the Laplace equation was apparently known already to Kelvin. Its explanation in terms of the null quadric first appeared in [3]. Null quadric as a tool to study conformal symmetries of the Laplace equation is the basic tool of $[15,17]$.

The conformal invariance of the Laplace equation generalizes to arbitrary pseudo-Riemannian manifolds. In fact, the Laplace-Beltrami operator plus an appropriate multiple of the scalar curvature, sometimes called the Yamabe Laplacian, is invariant in a generalized sense with respect to conformal maps. This can be found for instance in [37, 41].

The group of generalized symmetries of the heat equation was known already to Lie [24]. It was rediscovered (in the essentially equivalent context of the free Schrödinger equation) by Schrödinger [40]. It was then studied, e.g., in [12, 33]. Elementary notions from differential geometry used in our paper are well known. One of standard references in this subject is $[18,19]$.

A topic that is extensively treated in the literature on the relation of special functions to group theory, such as [27, 36, 43, 44, 45], is derivation of various addition formulas. Addition formulas say that a certain special function can be written as a sum, often infinite, of some related functions. These identities can be typically interpreted in terms of a certain representation of an appropriate Lie group. These identities are very interesting, however we do not discuss them. The only elements of Lie groups that we consider are very special - they are the "Weyl symmetries". They yield discrete symmetries of hypergeometric type equations, such as Kummer's table. We leave out addition formulas, because their theory is considerably more complicated than what we consider in our paper. 
The relationship of Kummer's table with the group of symmetries of a cube (which is the Weyl group of so $\left(\mathbb{C}^{6}\right)$ ) was discussed in [25]. A recent paper, where symmetries of the hypergeometric equation play an important role is [20]. (We learned the term "transmutation relations" from this paper).

The use of transmutation relations as a tool to derive recurrence relations for hypergeometric type functions is well known and can be found, e.g., in the book by Nikiforov-Uvarov [34], in the books by Miller [27] or in older works such as [42, 46, 47].

There exists various generalizations of hypergeometric type functions. Let us mention the class of $\mathcal{A}$-hypergeometric functions, which provides a natural generalization of the usual hypergeometric function to many-variable situations [2, 4]. Saito [39] considers generalized symmetries in the framework of $\mathcal{A}$-hypergeometric functions. Note, however, that the results of Saito are incomplete in the case of the classic hypergeometric equation. He admits this: "When $p=2$, the symmetry Lie algebra is much larger than $\mathfrak{g}_{2}$ ", and he quotes the paper by Miller [29]. Similarly, the (surprisingly large) Lie algebras of symmetries of the Gegenbauer and Hermite equations cannot be easily seen from a (seemingly very general) analysis of Saito.

There are a number of topics related to the hypergeometric type equation that we do not touch. Let us mention the question whether hypergeometric functions can be expressed in terms of algebraic functions. This topic, in the context of $\mathcal{A}$-hypergeometric functions was considered, e.g., in the interesting papers $[2,4]$.

In our paper we stick to a rather limited class of equations. We do not have the ambition to go for generalizations. This limited class has a surprisingly rich structure, which seems to be lost when we consider their generalizations.

Many, perhaps most identities and ideas described in our paper can be found in one form or another in the literature, especially in the works by Miller, also by Miller and Kalnins, as we discussed above. Nevertheless, we believe that our work raises important points that are not explicitly described in the literature. We argue that symmetry properties of all hypergeometric type equations become almost obvious if we add a certain number of variables obtaining the Laplace equation. We describe this idea in a unified framework, identifying the relationship of theory of hypergeometric type equations with such elements of group theory as roots, Cartan algebras and Weyl groups. These ideas are summed in Table 1, which to our knowledge has not appeared in the literature, except for the paper [6] written by one of us.

We use various (minor but helpful) ideas to make our presentation as short and transparent as possible: e.g., the balanced form of hypergeometric type equations, Lie algebraic parameters and split coordinates in $\mathbb{C}^{n}$. In our derivations the symmetries are completely obvious at the starting point, then at each step they become more and more complicated.

The derivation of generalized symmetries of the Laplacian, given after Theorem 6.4, is probably partly original. It leads to an interesting geometric object, which we call $\Delta^{\diamond}$. It satisfies identities (6.18) and (6.19), which seem quite important in the context of conformal invariance of the Laplace equation. These identities are elementary and quite simple, however we have never seen them in the literature. They can be used to derive factorizations of hypergeometric type equations, relating them to Casimir operators of certain distinguished subalgebras, another point that is probably original.

\section{Hypergeometric type operators and their symmetries}

In this section we describe the families of identities that we would like to interpret in a grouptheoretical fashion in this article. As mentioned above, all of them involve the balanced form of the operators (1.1)-(1.5). 


\subsection{Hypergeometric operator}

In the hypergeometric equation (1.1) we prefer to replace the parameters $a, b, c$ with

$$
\alpha:=c-1, \quad \beta:=a+b-c, \quad \mu:=b-a .
$$

We obtain the (standard) hypergeometric operator

$$
\begin{aligned}
\mathcal{F}_{\alpha, \beta, \mu}\left(w, \partial_{w}\right)= & w(1-w) \partial_{w}^{2}+((1+\alpha)(1-w)-(1+\beta) w) \partial_{w} \\
& +\frac{1}{4} \mu^{2}-\frac{1}{4}(\alpha+\beta+1)^{2} .
\end{aligned}
$$

Instead of (2.1) we prefer to consider the balanced hypergeometric operator

$$
\begin{aligned}
\mathcal{F}_{\alpha, \beta, \mu}^{\mathrm{bal}}\left(w, \partial_{w}\right) & :=w^{\frac{\alpha}{2}}(1-w)^{\frac{\beta}{2}} \mathcal{F}_{\alpha, \beta, \mu}\left(w, \partial_{w}\right)(1-w)^{-\frac{\beta}{2}} w^{-\frac{\alpha}{2}} \\
& =\partial_{w} w(1-w) \partial_{w}-\frac{\alpha^{2}}{4 w}-\frac{\beta^{2}}{4(1-w)}+\frac{\mu^{2}-1}{4} .
\end{aligned}
$$

Discrete symmetries. $\mathcal{F}_{\alpha, \beta, \mu}^{\text {bal }}\left(w, \partial_{w}\right)$ does not change if we flip the signs of $\alpha, \beta, \mu$. Besides, the following operators coincide with $\mathcal{F}_{\alpha, \beta, \mu}^{\text {bal }}\left(w, \partial_{w}\right)$ :

$$
\begin{array}{rlrl}
w & =z: & & \mathcal{F}_{\alpha, \beta, \mu}^{\mathrm{bal}}\left(z, \partial_{z}\right), \\
w=1-z: & & \mathcal{F}_{\beta, \alpha, \mu}^{\mathrm{bal}}\left(z, \partial_{z}\right), \\
w & =\frac{1}{z}: & & z^{\frac{1}{2}}(-z) \mathcal{F}_{\mu, \beta, \alpha}^{\mathrm{bal}}\left(z, \partial_{z}\right) z^{-\frac{1}{2}}, \\
w & =1-\frac{1}{z}: & & z^{\frac{1}{2}}(-z) \mathcal{F}_{\mu, \alpha, \beta}^{\mathrm{bal}}\left(z, \partial_{z}\right) z^{-\frac{1}{2}}, \\
w & =\frac{1}{1-z}: & & (1-z)^{\frac{1}{2}}(z-1) \mathcal{F}_{\beta, \mu, \alpha}^{\mathrm{bal}}\left(z, \partial_{z}\right)(1-z)^{-\frac{1}{2}}, \\
w & =\frac{z}{z-1}: & & (1-z)^{\frac{1}{2}}(z-1) \mathcal{F}_{\alpha, \mu, \beta}^{\mathrm{bal}}\left(z, \partial_{z}\right)(1-z)^{-\frac{1}{2}} .
\end{array}
$$

Transmutation relations:

$$
\begin{aligned}
& \sqrt{w(1-w)}\left(\partial_{w}-\frac{\alpha}{2 w}+\frac{\beta}{2(1-w)}\right) \mathcal{F}_{\alpha, \beta, \mu}^{\mathrm{bal}}\left(w, \partial_{w}\right) \\
& =\mathcal{F}_{\alpha+1, \beta+1, \mu}^{\mathrm{bal}}\left(w, \partial_{w}\right) \sqrt{w(1-w)}\left(\partial_{w}-\frac{\alpha}{2 w}+\frac{\beta}{2(1-w)}\right), \\
& \sqrt{w(1-w)}\left(\partial_{w}+\frac{\alpha}{2 w}-\frac{\beta}{2(1-w)}\right) \mathcal{F}_{\alpha, \beta, \mu}^{\mathrm{bal}}\left(w, \partial_{w}\right) \\
& =\mathcal{F}_{\alpha-1, \beta-1, \mu}^{\mathrm{bal}}\left(w, \partial_{w}\right) \sqrt{w(1-w)}\left(\partial_{w}+\frac{\alpha}{2 w}-\frac{\beta}{2(1-w)}\right), \\
& \sqrt{w(1-w)}\left(\partial_{w}-\frac{\alpha}{2 w}-\frac{\beta}{2(1-w)}\right) \mathcal{F}_{\alpha, \beta, \mu}^{\mathrm{bal}}\left(w, \partial_{w}\right) \\
& =\mathcal{F}_{\alpha+1, \beta-1, \mu}^{\mathrm{bal}}\left(w, \partial_{w}\right) \sqrt{w(1-w)}\left(\partial_{w}-\frac{\alpha}{2 w}-\frac{\beta}{2(1-w)}\right), \\
& \sqrt{w(1-w)}\left(\partial_{w}+\frac{\alpha}{2 w}+\frac{\beta}{2(1-w)}\right) \mathcal{F}_{\alpha, \beta, \mu}^{\mathrm{bal}}\left(w, \partial_{w}\right) \\
& =\mathcal{F}_{\alpha-1, \beta+1, \mu}^{\mathrm{bal}}\left(w, \partial_{w}\right) \sqrt{w(1-w)}\left(\partial_{w}+\frac{\alpha}{2 w}+\frac{\beta}{2(1-w)}\right),
\end{aligned}
$$




$$
\begin{aligned}
& \sqrt{w}\left(2(1-w) \partial_{w}-\frac{\alpha}{w}-\mu-3\right) \mathcal{F}_{\alpha, \beta, \mu}^{\mathrm{bal}}\left(w, \partial_{w}\right) \\
& =\mathcal{F}_{\alpha+1, \beta, \mu+1}^{\mathrm{bal}}\left(w, \partial_{w}\right) \sqrt{w}\left(2(1-w) \partial_{w}-\frac{\alpha}{w}-\mu-1\right), \\
& \sqrt{w}\left(2(1-w) \partial_{w}+\frac{\alpha}{w}+\mu-3\right) \mathcal{F}_{\alpha, \beta, \mu}^{\mathrm{bal}}\left(w, \partial_{w}\right) \\
& =\mathcal{F}_{\alpha-1, \beta, \mu-1}^{\mathrm{bal}}\left(w, \partial_{w}\right) \sqrt{w}\left(2(1-w) \partial_{w}+\frac{\alpha}{w}+\mu-1\right), \\
& \sqrt{w}\left(2(1-w) \partial_{w}-\frac{\alpha}{w}+\mu-3\right) \mathcal{F}_{\alpha, \beta, \mu}^{\mathrm{bal}}\left(w, \partial_{w}\right) \\
& =\mathcal{F}_{\alpha+1, \beta, \mu-1}^{\mathrm{bal}}\left(w, \partial_{w}\right) \sqrt{w}\left(2(1-w) \partial_{w}-\frac{\alpha}{w}+\mu-1\right), \\
& \sqrt{w}\left(2(1-w) \partial_{w}+\frac{\alpha}{w}-\mu-3\right) \mathcal{F}_{\alpha, \beta, \mu}^{\mathrm{bal}}\left(w, \partial_{w}\right) \\
& =\mathcal{F}_{\alpha-1, \beta, \mu+1}^{\mathrm{bal}}\left(w, \partial_{w}\right) \sqrt{w}\left(2(1-w) \partial_{w}+\frac{\alpha}{w}-\mu-1\right), \\
& \sqrt{1-w}\left(-2 w \partial_{w}-\frac{\beta}{1-w}-\mu-3\right) \mathcal{F}_{\alpha, \beta, \mu}^{\mathrm{bal}}\left(w, \partial_{w}\right) \\
& =\mathcal{F}_{\alpha, \beta+1, \mu+1}^{\mathrm{bal}}\left(w, \partial_{w}\right) \sqrt{1-w}\left(-2 w \partial_{w}-\frac{\beta}{1-w}-\mu-1\right), \\
& \sqrt{1-w}\left(-2 w \partial_{w}+\frac{\beta}{1-w}+\mu-3\right) \mathcal{F}_{\alpha, \beta, \mu}^{\mathrm{bal}}\left(w, \partial_{w}\right) \\
& =\mathcal{F}_{\alpha, \beta-1, \mu-1}^{\mathrm{bal}}\left(w, \partial_{w}\right) \sqrt{1-w}\left(-2 w \partial_{w}+\frac{\beta}{1-w}+\mu-1\right), \\
& \sqrt{1-w}\left(-2 w \partial_{w}-\frac{\beta}{1-w}+\mu-3\right) \mathcal{F}_{\alpha, \beta, \mu}^{\mathrm{bal}}\left(w, \partial_{w}\right) \\
& =\mathcal{F}_{\alpha, \beta+1, \mu-1}^{\mathrm{bal}}\left(w, \partial_{w}\right) \sqrt{1-w}\left(-2 w \partial_{w}-\frac{\beta}{1-w}+\mu-1\right), \\
& \sqrt{1-w}\left(-2 w \partial_{w}+\frac{\beta}{1-w}-\mu-3\right) \mathcal{F}_{\alpha, \beta, \mu}^{\mathrm{bal}}\left(w, \partial_{w}\right) \\
& =\mathcal{F}_{\alpha, \beta-1, \mu+1}^{\mathrm{bal}}\left(w, \partial_{w}\right) \sqrt{1-w}\left(-2 w \partial_{w}+\frac{\beta}{1-w}-\mu-1\right) .
\end{aligned}
$$

Factorizations:

$$
\begin{aligned}
\mathcal{F}_{\alpha, \beta, \mu}^{\mathrm{bal}}\left(w, \partial_{w}\right) \\
=\sqrt{w(1-w)}\left(\partial_{w}-\frac{\alpha-1}{2 w}+\frac{\beta-1}{2(1-w)}\right) \sqrt{w(1-w)}\left(\partial_{w}-\frac{\alpha}{2 w}+\frac{\beta}{2(1-w)}\right) \\
\quad-\frac{1}{4}\left((\beta+\alpha)(\beta+\alpha-2)+\mu^{2}-1\right) \\
=\sqrt{w(1-w)}\left(\partial_{w}+\frac{\alpha+1}{2 w}+\frac{\beta-1}{2(1-w)}\right) \sqrt{w(1-w)}\left(\partial_{w}-\frac{\alpha}{2 w}-\frac{\beta}{2(1-w)}\right) \\
\quad-\frac{1}{4}\left((\beta-\alpha)(\beta-\alpha-2)+\mu^{2}-1\right) \\
=\sqrt{w(1-w)}\left(\partial_{w}-\frac{\alpha-1}{2 w}-\frac{\beta+1}{2(1-w)}\right) \sqrt{w(1-w)}\left(\partial_{w}-\frac{\alpha}{2 w}-\frac{\beta}{2(1-w)}\right)
\end{aligned}
$$




$$
\begin{aligned}
& -\frac{1}{4}\left((\beta-\alpha)(\beta-\alpha+2)+\mu^{2}-1\right) \\
& =\sqrt{w(1-w)}\left(\partial_{w}+\frac{\alpha+1}{2 w}-\frac{\beta+1}{2(1-w)}\right) \sqrt{w(1-w)}\left(\partial_{w}-\frac{\alpha}{2 w}+\frac{\beta}{2(1-w)}\right) \\
& -\frac{1}{4}\left((\beta+\alpha)(\beta+\alpha+2)+\mu^{2}-1\right) \text {, } \\
& -(1-w) \mathcal{F}_{\alpha, \beta, \mu}^{\mathrm{bal}}\left(w, \partial_{w}\right) \\
& =-\sqrt{w}\left(-\frac{1}{2}+(1-w) \partial_{w}-\frac{\alpha-1}{2 w}-\frac{\mu-1}{2}\right) \sqrt{w}\left(-\frac{1}{2}+(1-w) \partial_{w}+\frac{\alpha}{2 w}+\frac{\mu}{2}\right) \\
& -\frac{1}{4}\left((\mu+\alpha)(\mu+\alpha-2)+\beta^{2}-1\right) \\
& =-\sqrt{w}\left(-\frac{1}{2}+(1-w) \partial_{w}+\frac{\alpha+1}{2 w}-\frac{\mu-1}{2}\right) \sqrt{w}\left(-\frac{1}{2}+(1-w) \partial_{w}-\frac{\alpha}{2 w}+\frac{\mu}{2}\right) \\
& -\frac{1}{4}\left((\mu-\alpha)(\mu-\alpha-2)+\beta^{2}-1\right) \\
& =-\sqrt{w}\left(-\frac{1}{2}+(1-w) \partial_{w}-\frac{\alpha-1}{2 w}+\frac{\mu+1}{2}\right) \sqrt{w}\left(-\frac{1}{2}+(1-w) \partial_{w}+\frac{\alpha}{2 w}-\frac{\mu}{2}\right) \\
& -\frac{1}{4}\left((\mu-\alpha)(\mu-\alpha+2)+\beta^{2}-1\right) \\
& =-\sqrt{w}\left(-\frac{1}{2}+(1-w) \partial_{w}+\frac{\alpha+1}{2 w}+\frac{\mu+1}{2}\right) \sqrt{w}\left(-\frac{1}{2}+(1-w) \partial_{w}-\frac{\alpha}{2 w}-\frac{\mu}{2}\right) \\
& -\frac{1}{4}\left((\mu+\alpha)(\mu+\alpha+2)+\beta^{2}-1\right) \text {, } \\
& -w \mathcal{F}_{\alpha, \beta, \mu}^{\mathrm{bal}}\left(w, \partial_{w}\right) \\
& =-\sqrt{1-w}\left(-\frac{1}{2}-w \partial_{w}-\frac{\beta-1}{2(1-w)}-\frac{\mu-1}{2}\right) \sqrt{1-w}\left(-\frac{1}{2}-w \partial_{w}+\frac{\beta}{2(1-w)}+\frac{\mu}{2}\right) \\
& -\frac{1}{4}\left((\mu+\beta)(\mu+\beta-2)+\alpha^{2}-1\right) \\
& =-\sqrt{1-w}\left(-\frac{1}{2}-w \partial_{w}+\frac{\beta+1}{2(1-w)}-\frac{\mu-1}{2}\right) \sqrt{1-w}\left(-\frac{1}{2}-w \partial_{w}-\frac{\beta}{2(1-w)}+\frac{\mu}{2}\right) \\
& -\frac{1}{4}\left((\mu-\beta)(\mu-\beta-2)+\alpha^{2}-1\right) \\
& =-\sqrt{1-w}\left(-\frac{1}{2}-w \partial_{w}-\frac{\beta-1}{2(1-w)}+\frac{\mu+1}{2}\right) \sqrt{1-w}\left(-\frac{1}{2}-w \partial_{w}+\frac{\beta}{2(1-w)}-\frac{\mu}{2}\right) \\
& -\frac{1}{4}\left((\mu-\beta)(\mu-\beta+2)+\alpha^{2}-1\right) \\
& =-\sqrt{1-w}\left(-\frac{1}{2}-w \partial_{w}+\frac{\beta+1}{2(1-w)}+\frac{\mu+1}{2}\right) \sqrt{1-w}\left(-\frac{1}{2}-w \partial_{w}-\frac{\beta}{2(1-w)}-\frac{\mu}{2}\right) \\
& -\frac{1}{4}\left((\mu+\beta)(\mu+\beta+2)+\alpha^{2}-1\right) \text {. }
\end{aligned}
$$

It is striking how symmetric the above formulas look like. The main goal of our paper is to explain why this is so.

\subsection{Gegenbauer operator}

In the hypergeometric equation (1.1) let us move the singular points to $-1,1$ and demand that it is reflection invariant. Then we can eliminate one of the parameters, say $c$. We obtain the 
Gegenbauer equation (1.2). We introduce new parameters

$$
\alpha:=\frac{a+b-1}{2}, \quad \lambda:=\frac{b-a}{2} .
$$

We obtain the (standard) Gegenbauer operator

$$
\mathcal{S}_{\alpha, \lambda}\left(w, \partial_{w}\right):=\left(1-w^{2}\right) \partial_{w}^{2}-2(1+\alpha) w \partial_{w}+\lambda^{2}-\left(\alpha+\frac{1}{2}\right)^{2} .
$$

The balanced Gegenbauer operator is

$$
\begin{aligned}
\mathcal{S}_{\alpha, \lambda}^{\mathrm{bal}}\left(w, \partial_{w}\right) & :=\left(w^{2}-1\right)^{\frac{\alpha}{2}} \mathcal{S}_{\alpha, \lambda}\left(w, \partial_{w}\right)\left(w^{2}-1\right)^{-\frac{\alpha}{2}} \\
& =\partial_{w}\left(1-w^{2}\right) \partial_{w}-\frac{\alpha^{2}}{1-w^{2}}+\lambda^{2}-\frac{1}{4} .
\end{aligned}
$$

Discrete symmetries. $\mathcal{S}_{\alpha, \lambda}^{\mathrm{bal}}\left(w, \partial_{w}\right)$ does not change if we flip the signs of $\alpha, \lambda$. Besides, the following operators coincide with $\mathcal{S}_{\alpha, \lambda}^{\mathrm{bal}}\left(w, \partial_{w}\right)$ :

$$
\begin{aligned}
& w=z: \quad \mathcal{S}_{\alpha, \lambda}^{\mathrm{bal}}\left(z, \partial_{z}\right), \\
& w=\frac{z}{\sqrt{z^{2}-1}}: \quad\left(z^{2}-1\right)^{\frac{1}{4}}\left(z^{2}-1\right) \mathcal{S}_{\lambda, \alpha}^{\mathrm{bal}}\left(z, \partial_{z}\right)\left(z^{2}-1\right)^{-\frac{1}{4}} .
\end{aligned}
$$

\section{Transmutation relations:}

$$
\begin{aligned}
& \sqrt{1-w^{2}}\left(-\frac{5}{2}-w \partial_{w}-\frac{\alpha}{1-w^{2}}-\lambda\right) \mathcal{S}_{\alpha, \lambda}^{\mathrm{bal}}\left(w, \partial_{w}\right) \\
& =\mathcal{S}_{\alpha+1, \lambda+1}^{\mathrm{bal}}\left(w, \partial_{w}\right) \sqrt{1-w^{2}}\left(-\frac{1}{2}-w \partial_{w}-\frac{\alpha}{1-w^{2}}-\lambda\right) \text {, } \\
& \sqrt{1-w^{2}}\left(-\frac{5}{2}-w \partial_{w}+\frac{\alpha}{1-w^{2}}+\lambda\right) \mathcal{S}_{\alpha, \lambda}^{\mathrm{bal}}\left(w, \partial_{w}\right) \\
& =\mathcal{S}_{\alpha-1, \lambda-1}^{\mathrm{bal}}\left(w, \partial_{w}\right) \sqrt{1-w^{2}}\left(-\frac{1}{2}-w \partial_{w}+\frac{\alpha}{1-w^{2}}+\lambda\right) \text {, } \\
& \sqrt{1-w^{2}}\left(-\frac{5}{2}-w \partial_{w}-\frac{\alpha}{1-w^{2}}+\lambda\right) \mathcal{S}_{\alpha, \lambda}^{\mathrm{bal}}\left(w, \partial_{w}\right) \\
& =\mathcal{S}_{\alpha+1, \lambda-1}^{\mathrm{bal}}\left(w, \partial_{w}\right) \sqrt{1-w^{2}}\left(-\frac{1}{2}-w \partial_{w}-\frac{\alpha}{1-w^{2}}+\lambda\right) \text {, } \\
& \sqrt{1-w^{2}}\left(-\frac{5}{2}-w \partial_{w}+\frac{\alpha}{1-w^{2}}-\lambda\right) \mathcal{S}_{\alpha, \lambda}^{\mathrm{bal}}\left(w, \partial_{w}\right) \\
& =\mathcal{S}_{\alpha-1, \lambda+1}^{\mathrm{bal}}\left(w, \partial_{w}\right) \sqrt{1-w^{2}}\left(-\frac{1}{2}-w \partial_{w}+\frac{\alpha}{1-w^{2}}-\lambda\right), \\
& w\left(-\frac{5}{2}+\frac{1-w^{2}}{w} \partial_{w}-\lambda\right) \mathcal{S}_{\alpha, \lambda}^{\mathrm{bal}}\left(w, \partial_{w}\right)=\mathcal{S}_{\alpha, \lambda+1}^{\mathrm{bal}}\left(w, \partial_{w}\right) w\left(-\frac{1}{2}+\frac{1-w^{2}}{w} \partial_{w}-\lambda\right), \\
& w\left(-\frac{5}{2}+\frac{1-w^{2}}{w} \partial_{w}+\lambda\right) \mathcal{S}_{\alpha, \lambda}^{\mathrm{bal}}\left(w, \partial_{w}\right)=\mathcal{S}_{\alpha, \lambda-1}^{\mathrm{bal}}\left(w, \partial_{w}\right) w\left(-\frac{1}{2}+\frac{1-w^{2}}{w} \partial_{w}+\lambda\right), \\
& \sqrt{1-w^{2}}\left(\partial_{w}+\frac{w}{1-w^{2}} \alpha\right) \mathcal{S}_{\alpha, \lambda}^{\mathrm{bal}}\left(w, \partial_{w}\right)=\mathcal{S}_{\alpha+1, \lambda}^{\mathrm{bal}}\left(w, \partial_{w}\right) \sqrt{1-w^{2}}\left(\partial_{w}+\frac{w}{1-w^{2}} \alpha\right), \\
& \sqrt{1-w^{2}}\left(\partial_{w}-\frac{w}{1-w^{2}} \alpha\right) \mathcal{S}_{\alpha, \lambda}^{\mathrm{bal}}\left(w, \partial_{w}\right)=\mathcal{S}_{\alpha+1, \lambda}^{\mathrm{bal}}\left(w, \partial_{w}\right) \sqrt{1-w^{2}}\left(\partial_{w}-\frac{w}{1-w^{2}} \alpha\right) .
\end{aligned}
$$




\section{Factorizations:}

$$
\begin{aligned}
& -\left(1-w^{2}\right) \mathcal{S}_{\alpha, \lambda}^{\mathrm{bal}}\left(w, \partial_{w}\right) \\
& =-w\left(\frac{1}{2}+\frac{1-w^{2}}{w} \partial_{w}-\lambda\right) w\left(-\frac{1}{2}+\frac{1-w^{2}}{w} \partial_{w}+\lambda\right)-\lambda^{2}+\lambda+\alpha^{2}-\frac{1}{4} \\
& =-w\left(\frac{1}{2}+\frac{1-w^{2}}{w} \partial_{w}+\lambda\right) w\left(-\frac{1}{2}+\frac{1-w^{2}}{w} \partial_{w}-\lambda\right)-\lambda^{2}-\lambda+\alpha^{2}-\frac{1}{4}, \\
& \mathcal{S}_{\alpha, \lambda}^{\mathrm{bal}}\left(w, \partial_{w}\right) \\
& =\sqrt{1-w^{2}}\left(\partial_{w}+\frac{w}{1-w^{2}}(\alpha-1)\right) \sqrt{1-w^{2}}\left(\partial_{w}-\frac{w}{1-w^{2}} \alpha\right) \\
& -\alpha^{2}+\alpha+\lambda^{2}-\frac{1}{4} \\
& =\sqrt{1-w^{2}}\left(\partial_{w}-\frac{w}{1-w^{2}}(\alpha+1)\right) \sqrt{1-w^{2}}\left(\partial_{w}+\frac{w}{1-w^{2}} \alpha\right)-\alpha^{2}-\alpha+\lambda^{2}-\frac{1}{4}, \\
& -w^{2} \mathcal{S}_{\alpha, \lambda}^{\mathrm{bal}}\left(w, \partial_{w}\right) \\
& =-\sqrt{1-w^{2}}\left(\frac{1}{2}-w \partial_{w}-\frac{\alpha-1}{1-w^{2}}-\lambda\right) \sqrt{1-w^{2}}\left(-\frac{1}{2}-w \partial_{w}+\frac{\alpha}{1-w^{2}}+\lambda\right) \\
& -(\lambda+\alpha)(\lambda+\alpha-2)-\frac{3}{4} \\
& =-\sqrt{1-w^{2}}\left(\frac{1}{2}-w \partial_{w}+\frac{\alpha+1}{1-w^{2}}-\lambda\right) \sqrt{1-w^{2}}\left(-\frac{1}{2}-w \partial_{w}-\frac{\alpha}{1-w^{2}}+\lambda\right) \\
& -(\lambda-\alpha)(\lambda-\alpha-2)-\frac{3}{4} \\
& =-\sqrt{1-w^{2}}\left(\frac{1}{2}-w \partial_{w}+\frac{\alpha+1}{1-w^{2}}+\lambda\right) \sqrt{1-w^{2}}\left(-\frac{1}{2}-w \partial_{w}-\frac{\alpha}{1-w^{2}}-\lambda\right) \\
& -(\lambda+\alpha)(\lambda+\alpha+2)-\frac{3}{4} \\
& =-\sqrt{1-w^{2}}\left(\frac{1}{2}-w \partial_{w}-\frac{\alpha-1}{1-w^{2}}+\lambda\right) \sqrt{1-w^{2}}\left(-\frac{1}{2}-w \partial_{w}+\frac{\alpha}{1-w^{2}}-\lambda\right) \\
& -(\lambda-\alpha)(\lambda-\alpha+2)-\frac{3}{4} \text {. }
\end{aligned}
$$

\subsection{Confluent operator}

In the confluent equation (1.3) we introduce new parameters

$$
\alpha:=c-1, \quad \theta:=2 a-c .
$$

The (standard) confluent operator is

$$
\mathcal{F}_{\theta, \alpha}\left(w, \partial_{w}\right):=w \partial_{w}^{2}+(1+\alpha-w) \partial_{w}-\frac{1}{2}(1+\theta+\alpha) .
$$

The balanced confluent operator is

$$
\mathcal{F}_{\theta, \alpha}^{\mathrm{bal}}\left(w, \partial_{w}\right):=w^{\frac{\alpha}{2}} \mathrm{e}^{-\frac{w}{2}} \mathcal{F}_{\theta, \alpha}\left(w, \partial_{w}\right) \mathrm{e}^{\frac{w}{2}} w^{-\frac{\alpha}{2}}=\partial_{w} w \partial_{w}-\frac{w}{4}-\frac{\theta}{2}-\frac{\alpha^{2}}{4 w} .
$$

Discrete symmetries. $\mathcal{F}_{\theta, \alpha}^{\mathrm{bal}}\left(w, \partial_{w}\right)$ does not change if we flip the sign of $\alpha$. Besides, the following operators coincide with $\mathcal{F}_{\theta, \alpha}^{\mathrm{bal}}\left(w, \partial_{w}\right)$ :

$$
w=z: \quad \mathcal{F}_{\theta, \alpha}^{\mathrm{bal}}\left(z, \partial_{z}\right), \quad w=-z: \quad \mathcal{F}_{-\theta, \alpha}^{\mathrm{bal}}\left(z, \partial_{z}\right) .
$$


Transmutation relations:

$$
\begin{aligned}
& \frac{1}{\sqrt{w}}\left(w \partial_{w}+\frac{\alpha}{2}+\frac{w}{2}\right) \mathcal{F}_{\theta, \alpha}^{\mathrm{bal}}\left(w, \partial_{w}\right)=\mathcal{F}_{\theta+1, \alpha-1}^{\mathrm{bal}}\left(w, \partial_{w}\right) \frac{1}{\sqrt{w}}\left(w \partial_{w}+\frac{\alpha}{2}+\frac{w}{2}\right), \\
& \frac{1}{\sqrt{w}}\left(w \partial_{w}-\frac{\alpha}{2}+\frac{w}{2}\right) \mathcal{F}_{\theta, \alpha}^{\mathrm{bal}}\left(w, \partial_{w}\right)=\mathcal{F}_{\theta+1, \alpha+1}^{\mathrm{bal}}\left(w, \partial_{w}\right) \frac{1}{\sqrt{w}}\left(w \partial_{w}-\frac{\alpha}{2}+\frac{w}{2}\right), \\
& \frac{1}{\sqrt{w}}\left(w \partial_{w}+\frac{\alpha}{2}-\frac{w}{2}\right) \mathcal{F}_{\theta, \alpha}^{\mathrm{bal}}\left(w, \partial_{w}\right)=\mathcal{F}_{\theta-1, \alpha-1}^{\mathrm{bal}}\left(w, \partial_{w}\right) \frac{1}{\sqrt{w}}\left(w \partial_{w}+\frac{\alpha}{2}-\frac{w}{2}\right), \\
& \frac{1}{\sqrt{w}}\left(w \partial_{w}-\frac{\alpha}{2}-\frac{w}{2}\right) \mathcal{F}_{\theta, \alpha}^{\mathrm{bal}}\left(w, \partial_{w}\right)=\mathcal{F}_{\theta-1, \alpha+1}^{\mathrm{bal}}\left(w, \partial_{w}\right) \frac{1}{\sqrt{w}}\left(w \partial_{w}-\frac{\alpha}{2}-\frac{w}{2}\right), \\
& \left(-w \partial_{w}-\frac{\theta}{2}-\frac{w}{2}-\frac{3}{2}\right) \mathcal{F}_{\theta, \alpha}^{\mathrm{bal}}\left(w, \partial_{w}\right)=\mathcal{F}_{\theta+2, \alpha}^{\mathrm{bal}}\left(w, \partial_{w}\right)\left(-w \partial_{w}-\frac{\theta}{2}-\frac{w}{2}-\frac{1}{2}\right), \\
& \left(w \partial_{w}-\frac{\theta}{2}-\frac{w}{2}+\frac{3}{2}\right) \mathcal{F}_{\theta, \alpha}^{\mathrm{bal}}\left(w, \partial_{w}\right)=\mathcal{F}_{\theta-2, \alpha}^{\mathrm{bal}}\left(w, \partial_{w}\right)\left(w \partial_{w}-\frac{\theta}{2}-\frac{w}{2}+\frac{1}{2}\right) .
\end{aligned}
$$

Factorizations:

$$
\begin{aligned}
-w \mathcal{F}_{\theta, \alpha}^{\mathrm{bal}}\left(w, \partial_{w}\right) & =\left(w \partial_{w}-\frac{\theta+1}{2}-\frac{w}{2}\right)\left(-w \partial_{w}-\frac{\theta+1}{2}-\frac{w}{2}\right)-\frac{1}{4}(\theta+1)^{2}+\frac{1}{4} \alpha^{2} \\
& =\left(-w \partial_{w}-\frac{\theta-1}{2}-\frac{w}{2}\right)\left(w \partial_{w}-\frac{\theta-1}{2}-\frac{w}{2}\right)-\frac{1}{4}(\theta-1)^{2}+\frac{1}{4} \alpha^{2}, \\
\mathcal{F}_{\theta, \alpha}^{\mathrm{bal}}\left(w, \partial_{w}\right) & =\frac{1}{\sqrt{w}}\left(w \partial_{w}-\frac{\alpha-1}{2}-\frac{w}{2}\right) \frac{1}{\sqrt{w}}\left(w \partial_{w}+\frac{\alpha}{2}+\frac{w}{2}\right)-\frac{1}{2}(\theta-\alpha+1) \\
& =\frac{1}{\sqrt{w}}\left(w \partial_{w}+\frac{\alpha+1}{2}+\frac{w}{2}\right) \frac{1}{\sqrt{w}}\left(w \partial_{w}-\frac{\alpha}{2}-\frac{w}{2}\right)-\frac{1}{2}(\theta-\alpha-1) \\
& =\frac{1}{\sqrt{w}}\left(w \partial_{w}+\frac{\alpha+1}{2}-\frac{w}{2}\right) \frac{1}{\sqrt{w}}\left(w \partial_{w}-\frac{\alpha}{2}+\frac{w}{2}\right)-\frac{1}{2}(\theta+\alpha+1) \\
& =\frac{1}{\sqrt{w}}\left(w \partial_{w}-\frac{\alpha-1}{2}+\frac{w}{2}\right) \frac{1}{\sqrt{w}}\left(w \partial_{w}+\frac{\alpha}{2}-\frac{w}{2}\right)-\frac{1}{2}(\theta+\alpha-1) .
\end{aligned}
$$

\subsection{Hermite operator}

In the Hermite equation (1.4) we prefer to use the parameter

$$
\lambda=a-\frac{1}{2} .
$$

The (standard) Hermite operator is

$$
\mathcal{S}_{\lambda}\left(w, \partial_{w}\right):=\partial_{w}^{2}-2 w \partial_{w}-2 \lambda-1 .
$$

The balanced Hermite operator is

$$
\mathcal{S}_{\lambda}^{\mathrm{bal}}\left(w, \partial_{w}\right):=\mathrm{e}^{-\frac{w^{2}}{2}} \mathcal{S}_{\lambda}\left(w, \partial_{w}\right) \mathrm{e}^{\frac{w^{2}}{2}}=\partial_{w}^{2}-w^{2}-2 \lambda
$$

Discrete symmetries. The following operators coincide with $\mathcal{S}_{\lambda}^{\text {bal }}\left(w, \partial_{w}\right)$ :

$$
\begin{array}{llll}
w=z: & \mathcal{S}_{\lambda}^{\mathrm{bal}}\left(z, \partial_{z}\right), & w=\mathrm{i} z: & -\mathcal{S}_{-\lambda}^{\mathrm{bal}}\left(z, \partial_{z}\right), \\
w=-z: & \mathcal{S}_{\lambda}^{\mathrm{bal}}\left(z, \partial_{z}\right), & w=-\mathrm{i} z: & -\mathcal{S}_{-\lambda}^{\mathrm{bal}}\left(z, \partial_{z}\right) .
\end{array}
$$


Transmutation relations:

$$
\begin{aligned}
& \left(\partial_{w}+w\right) \mathcal{S}_{\lambda}^{\mathrm{bal}}\left(w, \partial_{w}\right)=\mathcal{S}_{\lambda+1}^{\mathrm{bal}}\left(w, \partial_{w}\right)\left(\partial_{w}+w\right), \\
& \left(\partial_{w}-w\right) \mathcal{S}_{\lambda}^{\mathrm{bal}}\left(w, \partial_{w}\right)=\mathcal{S}_{\lambda-1}^{\mathrm{bal}}\left(w, \partial_{w}\right)\left(\partial_{w}-w\right), \\
& \left(-w \partial_{w}-\lambda-w^{2}-\frac{5}{2}\right) \mathcal{S}_{\lambda}^{\mathrm{bal}}\left(w, \partial_{w}\right)=\mathcal{S}_{\lambda+2}^{\mathrm{bal}}\left(w, \partial_{w}\right)\left(-w \partial_{w}-\lambda-w^{2}-\frac{1}{2}\right), \\
& \left(w \partial_{w}-\lambda-w^{2}+\frac{5}{2}\right) \mathcal{S}_{\lambda}^{\mathrm{bal}}\left(w, \partial_{w}\right)=\mathcal{S}_{\lambda-2}^{\mathrm{bal}}\left(w, \partial_{w}\right)\left(w \partial_{w}-\lambda-w^{2}+\frac{1}{2}\right) .
\end{aligned}
$$

Factorizations:

$$
\begin{array}{r}
-w^{2} \mathcal{S}_{\lambda}^{\mathrm{bal}}\left(w, \partial_{w}\right)=\left(w \partial_{w}-\lambda-\frac{3}{2}-w^{2}\right)\left(-w \partial_{w}-\lambda-\frac{1}{2}-w^{2}\right)-(\lambda+1)^{2}+\frac{1}{4} \\
=\left(-w \partial_{w}-\lambda+\frac{3}{2}-w^{2}\right)\left(w \partial_{w}-\lambda+\frac{1}{2}-w^{2}\right)-(\lambda-1)^{2}+\frac{1}{4} \\
\mathcal{S}_{\lambda}^{\mathrm{bal}}\left(w, \partial_{w}\right)=\left(\partial_{w}-w\right)\left(\partial_{w}+w\right)-2 \lambda-1=\left(\partial_{w}+w\right)\left(\partial_{w}-w\right)-2 \lambda+1 .
\end{array}
$$

\section{$2.5{ }_{0} F_{1}$ operator}

In the ${ }_{0} F_{1}$ equation (1.5) we prefer to use the parameter

$$
\alpha:=c-1 \text {. }
$$

The $(\text { standard })_{0} F_{1}$ operator is

$$
\mathcal{F}_{\alpha}\left(w, \partial_{w}\right):=w \partial_{w}^{2}+(\alpha+1) \partial_{w}-1 \text {. }
$$

The balanced ${ }_{0} F_{1}$ operator is

$$
\mathcal{F}_{\alpha}^{\mathrm{bal}}\left(w, \partial_{w}\right):=w^{\frac{\alpha}{2}} \mathcal{F}_{\alpha}\left(w, \partial_{w}\right) w^{-\frac{\alpha}{2}}=\partial_{w} w \partial_{w}-1-\frac{\alpha^{2}}{4 w} .
$$

Discrete symmetries. $\mathcal{F}_{\alpha}\left(w, \partial_{w}\right)$ does not change if we flip the sign of $\alpha$.

Transmutation relations:

$$
\begin{aligned}
& \frac{1}{\sqrt{w}}\left(w \partial_{w}-\frac{\alpha}{2}\right) \mathcal{F}_{\alpha}^{\mathrm{bal}}\left(w, \partial_{w}\right)=\mathcal{F}_{\alpha+1}^{\mathrm{bal}}\left(w, \partial_{w}\right) \frac{1}{\sqrt{w}}\left(w \partial_{w}-\frac{\alpha}{2}\right), \\
& \frac{1}{\sqrt{w}}\left(w \partial_{w}+\frac{\alpha}{2}\right) \mathcal{F}_{\alpha}^{\mathrm{bal}}\left(w, \partial_{w}\right)=\mathcal{F}_{\alpha-1}^{\mathrm{bal}}\left(w, \partial_{w}\right) \frac{1}{\sqrt{w}}\left(w \partial_{w}+\frac{\alpha}{2}\right) .
\end{aligned}
$$

Factorizations:

$$
\begin{aligned}
\mathcal{F}_{\alpha}^{\text {bal }}\left(w, \partial_{w}\right) & =\frac{1}{\sqrt{w}}\left(w \partial_{w}-\frac{\alpha-1}{2}\right) \frac{1}{\sqrt{w}}\left(w \partial_{w}+\frac{\alpha}{2}\right)-1 \\
& =\frac{1}{\sqrt{w}}\left(w \partial_{w}+\frac{\alpha+1}{2}\right) \frac{1}{\sqrt{w}}\left(w \partial_{w}-\frac{\alpha}{2}\right)-1 .
\end{aligned}
$$

\section{Basic complex geometry}

In this section we describe basic notation for complex geometry. Throughout the section, $\Omega, \Omega_{1}, \Omega_{2}$ are open subsets of $\mathbb{C}^{n}$ or, more generally, complex manifolds. We will write $\mathbb{C}^{\times}$ for the multiplicative group $\mathbb{C} \backslash\{0\}$.

We will write $\mathcal{A}(\Omega)$ for the set of holomorphic functions on $\Omega . y=\left(y^{1}, \ldots, y^{n}\right)$ will denote generic coordinates on $\Omega$. We will write $\mathcal{A}^{\times}(\Omega)$ for the set of nowhere vanishing holomorphic functions on $\Omega$. 


\subsection{Vector fields}

Let $\operatorname{hol}(\Omega)$ denote the Lie algebra of holomorphic vector fields on $\Omega$. Every $A \in \operatorname{hol}(\Omega)$ can be identified with the differential operator

$$
A f(y)=\sum_{i} A^{i}(y) \partial_{y^{i}} f(y), \quad f \in \mathcal{A}(\Omega)
$$

where $A^{i} \in \mathcal{A}(\Omega), i=1, \ldots, n$.

We will denote by $\mathcal{A} \rtimes \operatorname{hol}(\Omega)$ the Lie algebra of 1st order differential operators on $\Omega$ with holomorphic coefficents. Such operators can be written as

$$
(A+M) f(y):=\sum_{i} A^{i}(y) \partial_{y^{i}} f(y)+M(y) f(y)
$$

where $A \in \operatorname{hol}(\Omega)$ and $M \in \mathcal{A}(\Omega)$.

Let $\mathfrak{g}$ be a Lie subalgebra of $\operatorname{hol}(\Omega)$. A linear function $\mathfrak{g} \ni A \mapsto M_{A} \in \mathcal{A}(\Omega)$ satisfying

$$
A_{1} M_{A_{2}}-A_{2} M_{A_{1}}=M_{\left[A_{1}, A_{2}\right]}
$$

will be called a cocycle for $\mathfrak{g}$. Every cocycle together with $\eta \in \mathbb{C}$ determines a homomorphism

$$
\mathfrak{g} \ni A \mapsto A+\eta M_{A} \in \mathcal{A} \rtimes \operatorname{hol}(\Omega) .
$$

\subsection{Point transformations}

The set of biholomorphic maps $\Omega_{1} \rightarrow \Omega_{2}$ will be denoted $\operatorname{Hol}\left(\Omega_{1}, \Omega_{2}\right)$. We set $\operatorname{Hol}(\Omega):=$ $\operatorname{Hol}(\Omega, \Omega)$.

Let $\alpha \in \operatorname{Hol}\left(\Omega_{1}, \Omega_{2}\right)$. The transport of functions, vector fields, etc. by the map $\alpha$ will be also denoted by $\alpha$. More precisely, for $f \in \mathcal{A}\left(\Omega_{1}\right)$ we define $\alpha f \in \mathcal{A}\left(\Omega_{2}\right)$ by

$$
(\alpha f)(y):=f\left(\alpha^{-1}(y)\right)
$$

For $A \in \operatorname{hol}\left(\Omega_{1}\right), \alpha(A) \in \operatorname{hol}\left(\Omega_{2}\right)$ is defined as

$$
\alpha(A):=\alpha A \alpha^{-1}
$$

If $m \in \mathcal{A}^{\times}\left(\Omega_{2}\right)$, then we have a map $m \alpha: \mathcal{A}\left(\Omega_{1}\right) \rightarrow \mathcal{A}\left(\Omega_{2}\right)$ given by

$$
(m \alpha f)(y):=m(y) f\left(\alpha^{-1}(y)\right)
$$

$\mathcal{A}^{\times} \rtimes \operatorname{Hol}\left(\Omega_{1}, \Omega_{2}\right)$ will denote the set of transformations $\mathcal{A}\left(\Omega_{1}\right) \rightarrow \mathcal{A}\left(\Omega_{2}\right)$ of this form. Clearly, $\mathcal{A}^{\times} \rtimes \operatorname{Hol}(\Omega)$ is a group.

Suppose that $G$ is a subgroup of $\operatorname{Hol}(\Omega)$. A family $G \ni \alpha \mapsto m_{\alpha} \in \mathcal{A}^{\times}(\Omega)$ satisfying

$$
m_{\alpha_{2}}(y) m_{\alpha_{1}}\left(\alpha_{2}^{-1}(y)\right)=m_{\alpha_{2} \alpha_{1}}(y), \quad \alpha_{1}, \alpha_{2} \in G, \quad y \in \Omega,
$$

will be called a cocycle for $G$. Every cocycle together with $\eta \in \mathbb{Z}$ determines a homomorphism

$$
G \ni \alpha \mapsto m_{\alpha}^{\eta} \alpha \in \mathcal{A}^{\times} \rtimes \operatorname{Hol}(\Omega)
$$




\subsection{Local cocycles}

Unfortunately, the above definition of a cocycle on a group is too rigid for our purposes. Below we introduce a weaker version of this concept, which we will be better adapted to our goals.

As before, we assume that $G$ is a subgroup of $\operatorname{Hol}(\Omega)$. Besides, we fix $\Omega_{0}$ open in $\Omega$. For $\alpha \in G$ we will write

$$
\Omega_{0}^{\alpha}:=\Omega_{0} \cap \alpha\left(\Omega_{0}\right) .
$$

Furthermore, we suppose that to every $\alpha \in G$ we associate $m_{\alpha} \in \mathcal{A}^{\times}\left(\Omega_{0}^{\alpha}\right)$ satisfying

$$
m_{\alpha_{2}}(y) m_{\alpha_{1}}\left(\alpha_{2}^{-1}(y)\right)=m_{\alpha_{2} \alpha_{1}}(y), \quad \alpha_{1}, \alpha_{2} \in G, \quad y \in \Omega_{0} \cap \alpha_{2}\left(\Omega_{0}\right) \cap \alpha_{2} \circ \alpha_{1}\left(\Omega_{0}\right) .
$$

Then $G \mapsto m_{\alpha}$ will be called a local cocycle for $G$ based on $\Omega_{0}$.

Let $p \in \mathcal{A}^{\times}\left(\Omega_{0}\right)$. Then

$$
m_{\alpha}(y):=\frac{p(y)}{p\left(\alpha^{-1}(y)\right)}, \quad y \in \Omega_{0}^{\alpha}
$$

is a (trivial) example of a local cocycle based on $\Omega_{0}$. Note that if $p$ cannot be extended to a holomorphic function on the whole $\Omega$, then (3.3) cannot be extended to a true cocycle.

Let $\eta \in \mathbb{Z}$. For any $\alpha \in G$ we can define the map

$$
m_{\alpha}^{\eta} \alpha \in \mathcal{A}^{\times} \rtimes \operatorname{Hol}\left(\Omega_{0}^{\alpha^{-1}}, \Omega_{0}^{\alpha}\right) .
$$

For $\alpha_{1}, \alpha_{2} \in G$ and $\eta \in \mathbb{Z}$ we have the following weak form of the chain rule:

$$
\left(m_{\alpha_{2}}^{\eta} \alpha_{2} \circ m_{\alpha_{1}}^{\eta} \alpha_{1}\right)(y)=m_{\alpha_{2} \circ \alpha_{1}}^{\eta} \alpha_{2} \circ \alpha_{1}(y), \quad y \in \Omega_{0} \cap \alpha_{2}\left(\Omega_{0}\right) \cap \alpha_{2} \circ \alpha_{1}\left(\Omega_{0}\right) .
$$

It will be convenient have a special notation for such a collection of maps (3.4): We will write that

$$
G \ni \alpha \mapsto m_{\alpha}^{\eta} \alpha \underset{\text { loc }}{\in} \mathcal{A}^{\times} \rtimes \operatorname{Hol}\left(\Omega_{0}\right)
$$

is a local representation of $G$.

\subsection{Half-integer powers of a cocycle}

For non-integer exponents the power function is unfortunately multivalued. Because of that, strictly speaking, $\eta \notin \mathbb{Z}$ should not be allowed in (3.1). However, we will be forced to consider situations when $\eta$ is a half integer. This can be handled by the following formalism.

The non-identity element of the group $\mathbb{Z}_{2}$ acts on $\mathbb{C}^{\times}$by $\mathbb{C}^{\times} \ni a \mapsto-a \in \mathbb{C}^{\times}$. This defines $\mathbb{C}^{\times} / \mathbb{Z}_{2}$, which is the space of pairs of non-zero complex numbers differing by a sign.

Let $\eta \in \frac{1}{2}+\mathbb{Z}$. Then for any $a \in \mathbb{C}^{\times}$, the power $a^{\eta}$ can be interpreted as an element in $\mathbb{C}^{\times} / \mathbb{Z}_{2}$.

Let us restrict our attention to $\Omega$ that are simply connected. We then define

$$
\mathcal{A}^{\times}(\Omega) / \mathbb{Z}_{2}:=\left\{(f,-f): f \in \mathcal{A}^{\times}(\Omega)\right\},
$$

If $f \in \mathcal{A}^{\times}(\Omega)$, then $f^{\eta}$ is well defined as an element of $\mathcal{A}^{\times}(\Omega) / \mathbb{Z}_{2}$.

Remark 3.1. If $\Omega$ is not simply connected, then on the left hand side of (3.5) instead of $\Omega$ we need to put the double cover of $\Omega$. Then $f^{\eta}$ is still well defined. However we will not use this construction.

Let us go back to the setup of Section 3.2. We can then define $m_{\alpha}^{\eta} \in \mathcal{A}^{\times}(\Omega) / \mathbb{Z}_{2}$. Therefore, (3.1) can be interpreted as a group of transformations of $\mathcal{A}^{\times}(\Omega) / \mathbb{Z}_{2}$.

A similar remark applies to Section 3.3. 


\subsection{Generalized symmetries}

Let $\mathcal{C}$ be a linear differential operator on a complex manifold $\Omega$. Let $\alpha \in \operatorname{Hol}(\Omega)$. We say that it is a symmetry of $\mathcal{C}$ iff

$$
\alpha \mathcal{C}=\mathcal{C} \alpha .
$$

Let $m^{\sharp}, m^{b} \in \mathcal{A}^{\times}(\Omega)$. Define a pair of transformations in $\mathcal{A}^{\times} \rtimes \operatorname{Hol}(\Omega)$ :

$$
\alpha^{\sharp}:=m^{\sharp} \alpha, \quad \alpha^{b}:=m^{b} \alpha .
$$

We say that a pair $\left(\alpha^{\sharp}, \alpha^{b}\right)$ is a generalized symmetry of $\mathcal{C}$ if

$$
\alpha^{b} \mathcal{C}=\mathcal{C} \alpha^{\sharp}
$$

Clearly, the kernel of $\mathcal{C}$ is invariant wrt the action of $\alpha^{\sharp}$ :

$$
\mathcal{C} f=0 \quad \text { implies } \mathcal{C} \alpha^{\sharp} f=0 .
$$

Generalized symmetries of $\mathcal{C}$ form a group.

Let $A \in \operatorname{hol}(\Omega)$. We say that it is an infinitesimal symmetry of $\mathcal{C}$ iff

$$
A \mathcal{C}=\mathcal{C} A .
$$

Let $M^{\sharp}, M^{b} \in \mathcal{A}(\Omega)$. One can also consider a pair of operators in $\mathcal{A} \rtimes \operatorname{hol}(\Omega)$

$$
A^{\sharp}:=A+M^{\sharp}, \quad A^{b}:=A+M^{b} .
$$

We say that a pair $\left(A^{\sharp}, A^{b}\right)$ is a generalized infinitesimal symmetry of $\mathcal{C}$ if

$$
A^{b} \mathcal{C}=\mathcal{C} A^{\sharp} .
$$

Clearly, the kernel of $\mathcal{C}$ is invariant wrt the action of $A^{\sharp}$ :

$$
\mathcal{C} f=0 \quad \text { implies } \quad \mathcal{C} A^{\sharp} f=0 .
$$

Infinitesimal generalized symmetries of $\mathcal{C}$ form a Lie algebra.

\section{Line bundles}

\subsection{Scaling}

A holomorphic bundle $\pi: \mathcal{V} \rightarrow \mathcal{Y}$ is called a line bundle if its fibers are modelled on $\mathbb{C}^{\times} . \mathcal{V}$ is equipped with scaling, a homomorphism $\mathbb{C}^{\times} \ni s \mapsto \lambda_{s} \in \operatorname{Hol}(\mathcal{V})$ preserving the fibers, that is, satisfying $\pi \lambda_{s}=\pi$. The vector field obtained by differentiating $\lambda_{s}$ is called the vertical vector field and denoted $V$ :

$$
\left.\frac{\mathrm{d}}{\mathrm{d} s} \lambda_{s}\right|_{s=1}=: V
$$

For $v \in \mathcal{V}$, we will often simply write $s v$ instead of $\lambda_{s} v$. We will also write $s=\frac{\lambda_{s}(v)}{v}$.

Let $\mathcal{Y}_{0} \subset \mathcal{Y}$ be open. A section based on $\mathcal{Y}_{0}$ is a holomorphic map $\gamma: \mathcal{Y}_{0} \rightarrow \mathcal{V}$ such that $\pi \circ \gamma=\mathrm{id}$. Every section based on $\mathcal{Y}_{0}$ determines a trivialization of $\pi^{-1}\left(\mathcal{Y}_{0}\right)$

$$
\mathcal{Y}_{0} \times \mathbb{C}^{\times} \ni(y, s) \mapsto s \gamma(y) \in \pi^{-1}\left(\mathcal{Y}_{0}\right) .
$$




\subsection{Vector fields on a line bundle}

Let hol $^{\mathbb{C}^{\times}}(\mathcal{V})$ denote the Lie algebra of scaling invariant vector fields, that is,

$$
\operatorname{hol}^{\mathbb{C}^{\times}}(\mathcal{V}):=\left\{B \in \operatorname{hol}(\mathcal{V}): \lambda_{s} B=B \lambda_{s}, s \in \mathbb{C}^{\times}\right\} .
$$

Let $B \in \operatorname{hol}^{\mathbb{C}^{\times}}(\mathcal{V})$. Then $B$ determines a unique element of $\operatorname{hol}(\mathcal{Y})$, which will be denoted $B^{\diamond}$.

Let $\gamma$ be a section based on $\mathcal{Y}_{0}$. $B^{\diamond}$ can be transported by $\gamma$ onto $\gamma\left(\mathcal{Y}_{0}\right)$. Thus we obtain two vector fields on $\gamma\left(\mathcal{Y}_{0}\right):\left.B\right|_{\gamma\left(\mathcal{Y}_{0}\right)}$ and $\gamma\left(B^{\diamond}\right)$.

Proposition 4.1. For any $v \in \gamma\left(\mathcal{Y}_{0}\right), B(v)-\gamma\left(B^{\diamond}\right)(v)$ is parallel to $V(v)$. Therefore, there exists $M_{B}^{\gamma} \in \mathcal{A}\left(\mathcal{Y}_{0}\right)$ such that

$$
B(\gamma(y))=M_{B}^{\gamma}(y) V(\gamma(y))+\gamma\left(B^{\diamond}\right)(y), \quad y \in \mathcal{Y}_{0} .
$$

Moreover,

$$
\mathrm{hol}^{\mathbb{C}^{\times}}(\mathcal{V}) \ni B \mapsto M_{B}^{\gamma}
$$

is a cocycle. Hence, for any $\eta \in \mathbb{C}$,

$$
\operatorname{hol}^{\mathbb{C}^{\times}}(\mathcal{V}) \ni B \mapsto B^{\gamma, \eta}:=B^{\diamond}+\eta M_{B}^{\gamma} \in \mathcal{A} \rtimes \operatorname{hol}\left(\mathcal{Y}_{0}\right)
$$

is a representation of the Lie algebra of scaling invariant vector fields.

Proof. Every $B \in \operatorname{hol}\left(\mathcal{V}_{0}\right)$ can be written uniquely as

$$
B=\tilde{M}_{B}^{\gamma} V+B^{\gamma},
$$

where $\tilde{M}_{B}^{\gamma} \in \mathcal{A}\left(\mathcal{V}_{0}\right)$ and for any $s \in \mathbb{C}^{\times}$the vector field $\mathcal{Y}_{0} \ni y \mapsto B^{\gamma}(s \gamma(y))$ is tangent to the section $s \gamma\left(\mathcal{Y}_{0}\right)$. Assume now that $B \in \operatorname{hol}^{\mathbb{C}^{\times}}\left(\mathcal{V}_{0}\right)$. This means $[V, B]=0$, which is equivalent to

$$
\left(V \tilde{M}_{B}^{\gamma}\right) V+\left[V, B^{\gamma}\right]=0
$$

We also have

$$
\gamma\left(B^{\diamond}\right)(y)=B^{\gamma}(\gamma(y))
$$

and we set

$$
M_{B}^{\gamma}(y):=\tilde{M}_{B}^{\gamma}(\gamma(y)), \quad y \in \mathcal{Y}_{0} .
$$

Restricting (4.3) to $\gamma\left(\mathcal{Y}_{0}\right)$, using (4.5) and setting (4.6), we obtain (4.1).

Now let $B_{1}, B_{2} \in \operatorname{hol}\left(\mathcal{V}_{0}\right)$. Replacing $B$ in $(4.3)$ with $\left[B_{1}, B_{2}\right]$, we can write

$$
\left[B_{1}, B_{2}\right]=\tilde{M}_{\left[B_{1}, B_{2}\right]}^{\gamma} V+\left[B_{1}, B_{2}\right]^{\gamma} .
$$

If in addition $B_{1}, B_{2} \in \mathrm{hol}^{\mathbb{C}^{\times}}\left(\mathcal{V}_{0}\right)$, then using (4.4) with $B$ replaced with $B_{1}$ and $B_{2}$, we obtain

$$
\left[B_{1}, B_{2}\right]=\left(B_{1}^{\gamma} \tilde{M}_{B_{2}}^{\gamma}-B_{2}^{\gamma} \tilde{M}_{B_{1}}^{\gamma}\right) V+\left[B_{1}^{\gamma}, B_{2}^{\gamma}\right] .
$$

Comparing (4.8) with (4.7), we obtain

$$
B_{1}^{\gamma} \tilde{M}_{B_{2}}^{\gamma}-B_{2}^{\gamma} \tilde{M}_{B_{1}}^{\gamma}=\tilde{M}_{\left[B_{1}, B_{2}\right]}^{\gamma} .
$$

Restricting (4.9) to the section $\gamma\left(\mathcal{Y}_{0}\right)$, using (4.5) and setting (4.6), we obtain the cocycle relation

$$
B_{1}^{\diamond} M_{B_{2}}^{\gamma}-B_{2}^{\diamond} M_{B_{1}}^{\gamma}=M_{\left[B_{1}, B_{2}\right]}^{\gamma} .
$$




\subsection{Point transformations of a line bundle}

Let $\mathrm{Hol}^{\mathbb{C}^{\times}}(\mathcal{V})$ denote the group of scaling invariant biholomorphic maps of $\mathcal{V}$, that is

$$
\mathrm{Hol}^{\mathbb{C}^{\times}}(\mathcal{V}):=\left\{\alpha \in \operatorname{Hol}(\mathcal{V}): \alpha \lambda_{s}=\lambda_{s} \alpha, s \in \mathbb{C}^{\times}\right\}
$$

Let $\alpha \in \operatorname{Hol}^{\mathbb{C}^{\times}}(\mathcal{V})$. Then $\alpha$ determines a unique element of $\operatorname{Hol}(\mathcal{Y})$, which will be denoted by $\alpha^{\diamond}$.

Let $\gamma$ be a section over $\mathcal{Y}_{0}$. As in (3.2), we set $\mathcal{Y}_{0}^{\alpha^{\diamond}}:=\alpha^{\diamond}\left(\mathcal{Y}_{0}\right) \cap \mathcal{Y}_{0}$. We define $m_{\alpha}^{\gamma} \in \mathcal{A}\left(\mathcal{Y}_{0}^{\alpha^{\diamond}}\right)$ by

$$
m_{\alpha}^{\gamma}(y):=\frac{\gamma(y)}{\alpha \circ \gamma \circ\left(\alpha^{\diamond}\right)^{-1}(y)}, \quad y \in \mathcal{Y}_{0}^{\alpha^{\diamond}}
$$

\section{Proposition 4.2.}

$$
\operatorname{Hol}^{\mathbb{C}^{\times}}(\mathcal{V}) \ni \alpha \mapsto m_{\alpha}^{\gamma}
$$

is a cocycle. Hence for any $\eta \in \mathbb{Z}$

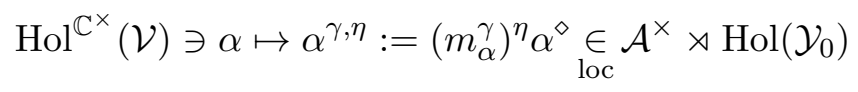

is a local representation.

\subsection{Homogeneous functions of integer degree}

As before, $\mathcal{Y}_{0} \subset \mathcal{Y}$ is open. We set $\mathcal{V}_{0}:=\pi^{-1}\left(\mathcal{Y}_{0}\right)$. For $\eta \in \mathbb{Z}$, let $\Lambda^{\eta}\left(\mathcal{V}_{0}\right)$ denote the space of holomorphic functions on $\mathcal{V}_{0}$ homogeneous of degree $\eta$, that is, functions $k \in \mathcal{A}\left(\mathcal{V}_{0}\right)$ satisfying

$$
k(s v)=s^{\eta} k(v), \quad v \in \mathcal{V}_{0}, \quad s \in \mathbb{C}^{\times} .
$$

Clearly, (4.11) implies

$$
V k=\eta k .
$$

Let $\gamma$ be a section based on $\mathcal{Y}_{0}$. We then have an obvious map $\psi^{\gamma, \eta}: \Lambda^{\eta}\left(\mathcal{V}_{0}\right) \rightarrow \mathcal{A}\left(\mathcal{Y}_{0}\right)$ : for $k \in \Lambda^{\eta}\left(\mathcal{V}_{0}\right)$ we set

$$
\left(\psi^{\gamma, \eta} k\right)(y):=k(\gamma(y)), \quad y \in \mathcal{Y}_{0}
$$

$\psi^{\gamma, \eta}$ is bijective and we can introduce its inverse, denoted $\phi^{\gamma, \eta}$, defined for any $f \in \mathcal{A}\left(\mathcal{Y}_{0}\right)$ by

$$
\left(\phi^{\gamma, \eta} f\right)(s \gamma(y))=s^{\eta} f(y), \quad s \in \mathbb{C}^{\times}, \quad y \in \mathcal{Y}_{0} .
$$

Proposition 4.3. With the notation of (4.2) and (4.10),

$$
\begin{aligned}
& B^{\gamma, \eta}:=\psi^{\gamma, \eta} B \phi^{\gamma, \eta} \in \mathcal{A} \rtimes \operatorname{hol}\left(\mathcal{Y}_{0}\right), \\
& \alpha^{\gamma, \eta}:=\psi^{\gamma, \eta} \alpha \phi^{\gamma, \eta} \in \mathcal{A}^{\times} \rtimes \operatorname{Hol}\left(\mathcal{Y}_{1}, \mathcal{Y}_{2}\right) .
\end{aligned}
$$




\subsection{Homogeneous functions of non-integer degree}

One can try to generalize the above construction to $\eta \in \mathbb{C} \backslash \mathbb{Z}$. In this case, there is a problem with the definition of functions homogeneous of degree $\eta$, because the power function is multivalued on $\mathbb{C}^{\times}$. Therefore, we cannot use $\mathcal{V}_{0}:=\pi^{-1}\left(\mathcal{Y}_{0}\right)$. Instead, let us we assume that $\mathcal{V}_{0} \subset \mathcal{V}$ is open, connected, $\pi\left(\mathcal{V}_{0}\right)=\mathcal{Y}_{0}$ and $\pi^{-1}(y) \cap \mathcal{V}_{0}$ is simply connected for any $y \in \mathcal{Y}_{0}$. We say that $k \in \Lambda^{\eta}\left(\mathcal{V}_{0}\right)$ if $k \in \mathcal{A}\left(\mathcal{V}_{0}\right)$ and

$$
k(s v)=s^{\eta} k(v), \quad v, s v \in \mathcal{V}_{0}, \quad s \in \mathbb{C}^{\times} .
$$

Note that (4.16) is unambiguous, because, for any $y \in \mathcal{Y}_{0}$, on $\pi^{-1}(y) \cap \mathcal{V}_{0}$ we have a unique continuation of holomorphic functions. (4.12) still holds.

Let $\gamma$ be a section based on $\mathcal{Y}_{0}$ whose image is contained in $\mathcal{V}_{0} \cdot \psi^{\gamma, \eta}$ is still bijective and we can introduce its inverse, denoted $\phi^{\gamma, \eta}$, defined for any $f \in \mathcal{A}(\mathcal{Y})$ by

$$
\left(\phi^{\gamma, \eta} f\right)(s \gamma(y))=s^{\eta} f(y), \quad s \in \mathbb{C}^{\times}, \quad y \in \mathcal{Y}_{0}, \quad s \gamma(y) \in \mathcal{V}_{0} .
$$

With this definition, (4.15) is still true.

\section{Complex Euclidean spaces}

\section{$5.1 \quad$ Linear transformations}

Let us first consider the vector space $\mathbb{C}^{n}$ without the Euclidean structure. The affine general linear Lie algebra $\mathbb{C}^{n} \rtimes \operatorname{gl}\left(\mathbb{C}^{n}\right)$ can be identified with the subalgebra of hol $\left(\mathbb{C}^{n}\right)$ spanned by

$$
\partial_{y^{j}}, \quad j=1, \ldots, n, \quad y^{i} \partial_{y^{j}}, \quad i, j=1, \ldots, n .
$$

Similarly, the affine general linear group $\mathbb{C}^{n} \rtimes \mathrm{GL}\left(\mathbb{C}^{n}\right)$ is a subgroup of $\operatorname{Hol}\left(\mathbb{C}^{n}\right)$.

We will have a special notation for the generator of dilations

$$
D_{\mathbb{C}^{n}}:=\sum_{i=1}^{n} y^{i} \partial_{y^{i}} .
$$

Obviously,

$$
\begin{array}{ll}
D_{\mathbb{C}^{n}} B=B D_{\mathbb{C}^{n}}, & B \in \operatorname{gl}\left(\mathbb{C}^{n}\right), \\
D_{\mathbb{C}^{n}} \alpha=\alpha D_{\mathbb{C}^{n}}, & \alpha \in \operatorname{GL}\left(\mathbb{C}^{n}\right) .
\end{array}
$$

Let $\sigma=\left(\sigma_{1}, \ldots, \sigma_{n}\right)$ be a permutation of $\{1, \ldots, n\}$. Then

$$
\sigma\left(y^{1}, \ldots, y^{n}\right):=\left(y^{\sigma_{1}^{-1}}, \ldots, y^{\sigma_{n}^{-1}}\right)
$$

defines an element of $\mathrm{GL}\left(\mathbb{C}^{n}\right)$. On the level of point transformations it acts as

$$
(\sigma f)\left(y^{1}, \ldots, y^{n}\right):=f\left(y^{\sigma_{1}}, \ldots, y^{\sigma_{n}}\right) .
$$

\subsection{Bilinear scalar product}

Suppose that

$$
\mathbb{C}^{n} \ni y, x \mapsto\langle y \mid x\rangle=\sum_{i, j} g_{i, j} y^{i} x^{j}
$$


is a nondegenerate symmetric bilinear form on $\mathbb{C}^{n}$ called the scalar product. Clearly, if we know the square of each vector

$$
\langle y \mid y\rangle=\sum_{i, j} g_{i, j} y^{i} y^{j}
$$

we have the complete information about the scalar product.

$\left[g^{i j}\right]$ will denote the inverse of $\left[g_{i j}\right]$. The orthogonal Lie algebra of $\mathbb{C}^{n}$, understood as a Lie subalgebra of $\operatorname{hol}\left(\mathbb{C}^{n}\right)$, is defined as

$$
\operatorname{so}\left(\mathbb{C}^{n}\right):=\left\{B \in \operatorname{gl}\left(\mathbb{C}^{n}\right): B\langle y \mid y\rangle=0\right\}
$$

For $i, j=1, \ldots, n$, define

$$
B_{i, j}:=\sum_{k}\left(g_{j, k} y_{i} \partial_{y_{k}}-g_{i, k} y_{k} \partial_{y_{j}}\right)
$$

$\left\{B_{i, j}: i<j\right\}$ is a basis of so $\left(\mathbb{C}^{n}\right)$. Clearly, $B_{i, j}=-B_{j, i}$, in particular $B_{i, i}=0$.

Likewise, recall that the orthogonal and the special orthogonal group of $\mathbb{C}^{n}$ is defined as

$$
\begin{aligned}
& \mathrm{O}\left(\mathbb{C}^{n}\right):=\left\{\alpha \in \mathrm{GL}\left(\mathbb{C}^{n}\right):\langle\alpha y \mid \alpha x\rangle=\langle y \mid x\rangle, y, x \in \mathbb{C}^{n}\right\}, \\
& \mathrm{SO}\left(\mathbb{C}^{n}\right):=\left\{\alpha \in \mathrm{O}\left(\mathbb{C}^{n}\right): \operatorname{det} \alpha=1\right\} .
\end{aligned}
$$

We define

$$
\begin{aligned}
& \text { the Laplacian } \Delta_{\mathbb{C}^{n}}:=\sum_{i, j=1}^{n} g^{i, j} \partial_{y^{i}} \partial_{y^{j}}, \quad \text { and } \\
& \text { the Casimir operator } \quad \mathcal{C}_{\mathbb{C}^{n}}:=\frac{1}{2} \sum_{i, j=1}^{n} g^{i, k} g^{j, l} B_{i, j} B_{k, l} .
\end{aligned}
$$

Clearly,

$$
\begin{array}{llll}
\Delta_{\mathbb{C}^{n}} B=B \Delta_{\mathbb{C}^{n}}, & B \in \mathbb{C}^{n} \rtimes \mathrm{so}\left(\mathbb{C}^{n}\right), & \mathcal{C}_{\mathbb{C}^{n}} B=B \mathcal{C}_{\mathbb{C}^{n}}, & B \in \mathrm{so}\left(\mathbb{C}^{n}\right), \\
\Delta_{\mathbb{C}^{n}} \alpha=\alpha \Delta_{\mathbb{C}^{n}}, & \alpha \in \mathbb{C}^{n} \rtimes \mathrm{O}\left(\mathbb{C}^{n}\right), & \mathcal{C}_{\mathbb{C}^{n}} \alpha=\alpha \mathcal{C}_{\mathbb{C}^{n}}, \quad \alpha \in \mathrm{O}\left(\mathbb{C}^{n}\right) .
\end{array}
$$

Note the identity

$$
\Delta_{\mathbb{C}^{n}}=\frac{1}{\langle y \mid y\rangle}\left(D_{\mathbb{C}^{n}}^{2}+(n-2) D_{\mathbb{C}^{n}}+\mathcal{C}_{\mathbb{C}^{n}}\right)
$$

We will denote by $\mathcal{S}^{n-1}(R)$ the (complex) sphere in $\mathbb{C}^{n}$ of squared radius $R$, that is

$$
\mathcal{S}^{n-1}(R):=\left\{y \in \mathbb{C}^{n}:\langle y \mid y\rangle=R\right\}
$$

We also introduce the null quadric

$$
\mathcal{V}^{n-1}:=\mathcal{S}^{n-1}(0) \backslash\{0\}
$$




\section{$5.3 \quad$ Split coordinates}

The coordinates that we describe in this subsection are particularly convenient for the analysis of $\operatorname{so}\left(\mathbb{C}^{n}\right)$ and $\mathrm{O}\left(\mathbb{C}^{n}\right)$. Let $n=2 m$ if $n$ is even and $n=2 m+1$ if $n$ is odd. Set

$$
I_{n}:= \begin{cases}\{-1,1, \ldots,-m, m\}, & \text { for even } n, \\ \{0,-1,1, \ldots,-m, m\}, & \text { for odd } n .\end{cases}
$$

The coordinates in $\mathbb{C}^{n}$ will be labelled by $I_{n}$, so that the square of $y=\left[y^{i}\right]_{i \in I_{n}}$ is given by

$$
\langle y \mid y\rangle=\sum_{i \in I_{n}} y_{i} y_{-i}= \begin{cases}\sum_{i=1}^{m} 2 y_{-i} y_{i}, & \text { for even } n \\ y_{0}^{2}+\sum_{i=1}^{m} 2 y_{-i} y_{i} & \text { for odd } n .\end{cases}
$$

Clearly, $g_{i, j}=g^{i, j}=\delta_{i,-j}$.

For $n=2 m, \operatorname{so}\left(\mathbb{C}^{n}\right)$ has a basis consisting of

$$
\begin{array}{ll}
N_{i}:=B_{i-i}=y_{-i} \partial_{y_{-i}}-y_{i} \partial_{y_{i}}, & j=1, \ldots, m, \\
B_{i j}:=y_{-i} \partial_{y_{j}}-y_{-j} \partial_{y_{i}}, & 1 \leq|i|<|j| \leq m .
\end{array}
$$

For $n=2 m+1$ we have to add

$$
B_{0 j}=y_{0} \partial_{y_{j}}-y_{-j} \partial_{y_{0}}, \quad|j|=1, \ldots, m \text {. }
$$

The subalgebra of so $\left(\mathbb{C}^{n}\right)$ spanned by (5.3) is its Cartan algebra. (5.4), and in the odd case also (5.5), are its root operators:

$$
\left[N_{k}, B_{i, j}\right]=\left(\operatorname{sgn}(i) \delta_{k,|i|}+\operatorname{sgn}(j) \delta_{k,|j|}\right) B_{i, j}, \quad\left[N_{k}, B_{0, j}\right]=\operatorname{sgn}(j) \delta_{k,|j|} B_{i, j} .
$$

We have

$$
\Delta_{\mathbb{C}^{n}}=\sum_{i \in I_{n}} \partial_{y_{i}} \partial_{y_{-i}}, \quad \mathcal{C}_{\mathbb{C}^{n}}=\frac{1}{2} \sum_{i, j \in I_{n}} B_{i, j} B_{-i,-j}
$$

\section{$5.4 \quad$ Weyl symmetries}

In our applications of the group invariance we will restrict ourselves only to the so-called "Weyl symmetries". It will be convenient to consider "Weyl symmetries" contained not only in $\mathrm{SO}\left(\mathbb{C}^{n}\right)$, but in the whole $\mathrm{O}\left(\mathbb{C}^{n}\right)$.

Permutations of $I_{2 m}$ that preserve its decomposition into pairs $\{-1,1\}, \ldots,\{-m, m\}$ correspond to a subgroup of $\mathrm{O}(2 m)$ that will be denoted $\mathrm{W}\left(\mathbb{C}^{2 m}\right)$. It is isomorphic to $\mathbb{Z}_{2}^{m} \rtimes S_{m}$. It is generated by two kinds of transformations: $\tau_{j}, j=1, \ldots, m$, which swap the elements of the $j$ th pair, and permutations from $S_{m}$, which permute the pairs. If $\sigma=\left(\sigma_{1}, \ldots, \sigma_{m}\right) \in S_{m}$, then

$$
\sigma f\left(y_{-1}, y_{1}, \ldots, y_{-m}, y_{m}\right):=f\left(y_{-\sigma_{1}}, y_{\sigma_{1}}, \ldots, y_{-\sigma_{m}}, y_{\sigma_{m}}\right)
$$

For $j=1, \ldots, n$,

$$
\tau_{j} f\left(y_{-1}, y_{1}, \ldots, y_{-j}, y_{j}, \ldots\right):=f\left(y_{-1}, y_{1}, \ldots, y_{j}, y_{-j}, \ldots\right)
$$


We have

$$
\begin{array}{ll}
\sigma B_{i, j} \sigma^{-1}=B_{\sigma_{i}, \sigma_{j}}, & \tau_{k} B_{i, j} \tau_{k}^{-1}=(-1)^{\delta_{|i|, k}+\delta_{|j|, k}} B_{i, j}, \\
\sigma N_{j} \sigma^{-1}=N_{\sigma_{j}}, & \tau_{k} N_{j} \tau_{k}^{-1}=(-1)^{\delta_{j, k}} N_{j} .
\end{array}
$$

Using $\mathbb{C}^{2 m+1}=\mathbb{C} \oplus \mathbb{C}^{2 m}$, we embed $\mathrm{W}\left(\mathbb{C}^{2 m}\right)$ in $\mathrm{O}\left(\mathbb{C}^{2 m+1}\right)$. We also introduce a transformation $\tau_{0} \in \mathrm{O}\left(\mathbb{C}^{2 m+1}\right)$ given by

$$
\tau_{0} f\left(y_{0}, y_{-1}, y_{1}, \ldots, y_{-m}, y_{m}\right):=f\left(-y_{0}, y_{-1}, y_{1}, \ldots, y_{-m}, y_{m}\right) .
$$

Clearly, $\tau_{0}$ commutes with $\mathrm{W}\left(\mathbb{C}^{2 m}\right)$. The group $\mathrm{W}\left(\mathbb{C}^{2 m+1}\right)$ is defined as the group generated by $\mathrm{W}\left(\mathbb{C}^{2 m}\right)$ and $\tau_{0}$, and is isomorphic to $\mathbb{Z}_{2} \times \mathbb{Z}_{2}^{m} \rtimes S_{m}$. We have for $i, j=1, \ldots, m$

$$
\tau_{0} B_{0, j} \tau_{0}^{-1}=-B_{0, j}, \quad \tau_{0} B_{i, j} \tau_{0}^{-1}=B_{i, j}, \quad \tau_{0} N_{j} \tau_{0}^{-1}=N_{j} .
$$

In both even and odd cases $\mathrm{W}\left(\mathbb{C}^{n}\right)$ acts as a group of automorphisms of $\operatorname{so}\left(\mathbb{C}^{n}\right)$ leaving invariant the Cartan algebra. To compute the determinant of elements of $\mathrm{W}\left(\mathbb{C}^{n}\right)$ it suffices to remember that $\operatorname{det} \sigma=1$ for $\sigma \in S_{m}$ and $\operatorname{det} \tau_{j}=-1$.

\section{Conformal invariance}

The main subject of this section is the description of generalized (infinitesimal) symmetries of the Laplace equation

$$
\Delta_{\mathbb{C}^{n}} f=0 .
$$

We will see in particular that the Lie algebra of generalized symmetries is so $\left(\mathbb{C}^{n+2}\right)$. We will see that it is convenient to start the description of these symmetries from the space $\mathbb{C}^{n+2}$, which

we will call the extended space. The space $\mathbb{C}^{n}$ will be embedded inside $\mathbb{C}^{n+2}$ as a section of the null quadric. We will see how the Laplacian $\Delta_{\mathbb{C}^{n+2}}$ reduces to the Laplacian $\Delta_{\mathbb{C}^{n}}$.

\subsection{Conformal invariance of Riemannian manifolds}

Suppose that a (complex) manifold $\Omega$ is equipped with a nondegenerate holomorphic covariant 2 -tensor field $g$, called the (complex) metric tensor. We will say that $(\Omega, g)$ is a (complex) Riemannian space.

Thus if $A, B \in \operatorname{hol}(\Omega)$, then we have a holomorphic function

$$
\Omega \ni y \mapsto g(A, B)(y)=g_{i, j}(y) A^{i}(y) B^{j}(y)
$$

called the scalar product.

Let $\alpha \in \operatorname{Hol}(\Omega)$. We can transport $g$ by $\alpha$ :

$$
\alpha(g)(A, B):=g\left(\alpha^{-1}(A), \alpha^{-1}(B)\right) .
$$

We say that $\alpha$ is conformal if there exists $m_{\alpha} \in \mathcal{A}^{\times}(\Omega)$ such that

$$
\alpha(g)=m_{\alpha} g .
$$

Let $\operatorname{Cf}(\Omega)$ denote the group of conformal maps on $(\Omega, g)$. One can check that

$$
\mathrm{Cf}(\Omega) \times \Omega \ni(\alpha, y) \mapsto m_{\alpha}(y) \in \mathcal{A}^{\times}(\Omega)
$$

is a cocycle. 
Let $C \in \operatorname{hol}(\Omega)$. The Lie derivative of $g$ in the direction of $C$ is denoted $C g$ and defined by

$$
(C g)(A, B):=C(g(A, B))-g([C, A], B)-g(A,[C, B]) .
$$

We say that $C$ is infinitesimally conformal if there exists $M_{C} \in \mathcal{A}(\Omega)$ such that

$$
C g=M_{C} g \text {. }
$$

Let $\operatorname{cf}(\Omega)$ denote the Lie algebra of infinitesimally conformal fields. One can check that

$$
\operatorname{cf}(\Omega) \times \Omega \ni(C, y) \mapsto M_{C}(y) \in \mathcal{A}(\Omega)
$$

is a cocycle.

We say that a manifold $\Omega$ has a conformal structure, if it is covered by a family of open sets $\Omega_{i}$ equipped with bilinear scalar products $g_{i}$ such that on $\Omega_{i} \cap \Omega_{j}$ we have

$$
g_{i}=\rho_{i, j} g_{j}
$$

for some $\rho_{i, j} \in \mathcal{A}^{\times}\left(\Omega_{i} \cap \Omega_{j}\right)$. Clearly, a Riemannian structure on $\Omega$ is not necessary to define $\operatorname{Cf}(\Omega)$ and $\operatorname{cf}(\Omega)$ - we need only a conformal structure on $\Omega$.

\subsection{Null quadric}

Consider the extended space, that is, the complex Euclidean space $\mathbb{C}^{n+2}$. The central role will be played by the representations

$$
\begin{aligned}
& \mathrm{so}\left(\mathbb{C}^{n+2}\right) \ni B \mapsto B \in \operatorname{hol}\left(\mathbb{C}^{n+2}\right), \\
& \mathrm{O}\left(\mathbb{C}^{n+2}\right) \ni \alpha \mapsto \alpha \in \operatorname{Hol}\left(\mathbb{C}^{n+2}\right),
\end{aligned}
$$

and the symmetry

$$
\begin{array}{ll}
B \Delta_{\mathbb{C}^{n+2}}=\Delta_{\mathbb{C}^{n+2}} B, & B \in \operatorname{so}\left(\mathbb{C}^{n+2}\right), \\
\alpha \Delta_{\mathbb{C}^{n+2}}=\Delta_{\mathbb{C}^{n+2}} \alpha, & \alpha \in \mathrm{O}\left(\mathbb{C}^{n+2}\right) .
\end{array}
$$

As in (5.2), we introduce

$$
\mathcal{V}:=\left\{z \in \mathbb{C}^{n+2}: z \neq 0,\langle z \mid z\rangle=0\right\}
$$

called the null quadric.

Multiplication by $s \in \mathbb{C}^{\times}$preserves $\mathcal{V}$. Therefore, we can define the projective quadric

$$
\mathcal{Y}:=\mathcal{V} / \mathbb{C}^{\times}=\left\{\mathbb{C}^{\times} z: z \in \mathcal{V}\right\}
$$

It is an $n$-dimensional complex manifold. Let $\pi: \mathcal{V} \rightarrow \mathcal{Y}$ denote the natural projection. Clearly, $\mathcal{V}$ is a complex line bundle over $\mathcal{Y}$. As in Section 4.1, the multiplication by $s$ will be often denoted by $\mathbb{C}^{\times} \ni s \mapsto \lambda_{s} \in \operatorname{Hol}(\mathcal{V})$ and the corresponding vertical vector field by $V \in \operatorname{hol}(\mathcal{V})$.

We can restrict (6.2) and (6.3) to $\mathcal{V}$ and note that they are scaling invariant. Thus, we have natural embeddings

$$
\begin{aligned}
& \operatorname{so}\left(\mathbb{C}^{n+2}\right) \ni B \mapsto B \in \mathrm{hol}^{\mathbb{C}^{\times}}(\mathcal{V}), \\
& \mathrm{O}\left(\mathbb{C}^{n+2}\right) \ni \alpha \mapsto \alpha \in \mathrm{Hol}^{\mathbb{C}^{\times}}(\mathcal{V}) .
\end{aligned}
$$

(Recall that hol ${ }^{\mathbb{C}^{\times}}(\mathcal{V})$, resp. $\mathrm{Hol}^{\mathbb{C}^{\times}}(\mathcal{V})$ denote the scaling invariant holomorphic vector fields, resp. bijections). Therefore, (6.6) and (6.7) induce their actions on $\mathcal{Y}$ :

$$
\begin{aligned}
& \operatorname{so}\left(\mathbb{C}^{n+2}\right) \ni B \mapsto B^{\diamond} \in \operatorname{hol}(\mathcal{Y}), \\
& \mathrm{O}\left(\mathbb{C}^{n+2}\right) \ni \alpha \mapsto \alpha^{\diamond} \in \operatorname{Hol}(\mathcal{Y}) .
\end{aligned}
$$




\subsection{Conformal invariance of projective quadric}

Let $g$ denote the restriction of the metric tensor on $\mathbb{C}^{n+2}$ to $\mathcal{V}$. Note that the null space of $g$ is 1-dimensional and is spanned by the vertical field $V$. In particular,

$$
g(V, A)=0, \quad A \in \operatorname{hol}(\mathcal{V}) .
$$

Moreover, the scaling scales the metric tensor:

$$
\begin{aligned}
& \lambda_{s}(g)=s^{2} g, \quad s \in \mathbb{C}^{\times}, \\
& V g=2 g .
\end{aligned}
$$

Using (6.10), we can extend (6.11) to multiplication by nonconstant functions:

Proposition 6.1. Let $\mathcal{U}$ be open in $\mathcal{Y}, m \in \mathcal{A}^{\times}(\mathcal{U})$. Define $\lambda_{m} \in \operatorname{Hol}\left(\pi^{-1}(\mathcal{U})\right)$ by

$$
\lambda_{m}(z):=m(\pi(z)) z, \quad z \in \pi^{-1}(\mathcal{U}) .
$$

Then $\lambda_{m} g=m^{2} g$. In particular, for any section $\gamma$, the restriction $\lambda_{m}: \gamma(\mathcal{U}) \rightarrow m \circ \pi \gamma(\mathcal{U})$ is conformal.

Let $\mathcal{U}$ be open in $\mathcal{Y}$. Let $\gamma$ be a section over $\mathcal{U}$. The tensor $g$ restricted to $\gamma(\mathcal{U})$ is nondegenerate. We can transport it by $\gamma^{-1}$ onto $\mathcal{U}$. This way we endow $\mathcal{U}$ with a metric tensor.

For $i=1,2$, let $\mathcal{U}_{i}$ be two open subsets of $\mathcal{Y}$ equipped with sections $\gamma_{i}$. Let $g_{i}$ be the corresponding complex Riemannian tensors. Obviously, there exists $\rho_{2,1} \in \mathcal{A}^{\times}\left(\mathcal{U}_{1} \cap \mathcal{U}_{2}\right)$ such that

$$
\gamma_{2}(y)=\rho_{2,1}(y) \gamma_{1}(y), \quad y \in \mathcal{U}_{1} \cap \mathcal{U}_{2}
$$

Therefore, by Proposition 6.1,

$$
g_{2}=\left(\gamma_{2}^{-1} \gamma_{1}\right) g_{1}=\rho_{2,1}^{2} g_{1}
$$

Cover $\mathcal{Y}$ with open subsets $\mathcal{U}_{i}, i=1, \ldots, N$, equipped with sections $\gamma_{i}$. Let $g_{i}$ be the corresponding Riemannian tensors on $\mathcal{U}_{i}$. Then on $\mathcal{U}_{i} \cap \mathcal{U}_{j}$ we have

$$
g_{j}=\rho_{j, i}^{2} g_{i}, \quad \rho_{j, i} \in \mathcal{A}^{\times}\left(\mathcal{U}_{i} \cap \mathcal{U}_{j}\right) .
$$

This way we endow $\mathcal{Y}$ with a conformal structure. It is easy to see that it does not depend on the choice of the covering and sections.

Proposition 6.2. (6.8) is infinitesimally conformal and (6.9) is conformal.

Proof. Let $\gamma$ be a section over $\mathcal{U} \subset \mathcal{Y}$.

Let $B \in \operatorname{so}\left(\mathbb{C}^{n+2}\right)$. By Proposition 4.1 , there exists $M_{B}^{\gamma} \in \mathcal{A}(\mathcal{U})$ such that

$$
\gamma\left(B^{\diamond}\right)=\left.\left(B-M_{B}^{\gamma} V\right)\right|_{\gamma(\mathcal{U})} .
$$

Now $B g=0$ and $V g=2 g$. Hence

$$
\gamma\left(B^{\diamond}\right) g=-2 M_{B}^{\gamma} g \text {. }
$$

Therefore, $\gamma\left(B^{\diamond}\right)$ is infinitesimally conformal on $\gamma(\mathcal{U})$. Hence $B^{\diamond}$ is infinitesimally conformal on $\mathcal{U}$. 
Let $\alpha \in \mathrm{O}\left(\mathbb{C}^{n+2}\right)$. Clearly, $\gamma_{\alpha}:=\alpha \circ \gamma \circ\left(\alpha^{\diamond}\right)^{-1}$ is a section based on $\alpha^{\diamond}(\mathcal{U})$. Therefore, there exists $m \in \mathcal{A}^{\times}\left(\alpha^{\diamond}(\mathcal{U}) \cap \mathcal{U}\right)$ such that

$$
\gamma(y)=m(y) \gamma_{\alpha}(y), \quad y \in \alpha^{\diamond}(\mathcal{U}) \cap \mathcal{U}
$$

Define now $\beta: \pi^{-1}\left(\mathcal{U} \cap\left(\alpha^{\diamond}\right)^{-1}(\mathcal{U})\right) \rightarrow \pi^{-1}\left(\alpha^{\diamond}(\mathcal{U}) \cap \mathcal{U}\right)$

$$
\beta(z):=m(\pi(z)) \alpha(z) .
$$

Substituting $z=\gamma(y)$ and using (6.13) we obtain

$$
\beta(\gamma(y))=m(y) \alpha(\gamma(y))=\gamma\left(\alpha^{\diamond}(y)\right)
$$

Thus $\beta$ maps $\gamma\left(\mathcal{U} \cap\left(\alpha^{\diamond}\right)^{-1}(\mathcal{U})\right)$ onto $\gamma\left(\alpha^{\diamond}(\mathcal{U}) \cap \mathcal{U}\right)$. Let $\beta^{\gamma}$ denote this restriction of $\beta$. By Proposition $6.1, \beta^{\gamma}$ is conformal. Clearly, it satisfies the identity

$$
\beta^{\gamma}=\gamma \alpha^{\diamond} \gamma^{-1}
$$

Hence $\alpha^{\diamond}$ is conformal.

\subsection{Conformal invariance of complex Euclidean space}

Fix a vector $e \in \mathcal{V}$, and set

$$
\mathcal{V}_{e}:=\{z \in \mathcal{V}:\langle z \mid e\rangle \neq 0\}
$$

Clearly, $\mathcal{V}_{e}$ is invariant with respect to the action of $\mathbb{C}^{\times}$and $\mathcal{Y}_{e}:=\mathcal{V}_{e} / \mathbb{C}^{\times}$is an open dense subset of $\mathcal{Y}$. We have a natural section of the line bundle $\mathcal{V} \rightarrow \mathcal{Y}$ based on $\mathcal{Y}_{e}$ :

$$
\mathcal{Y}_{e} \ni \mathbb{C}^{\times} z \mapsto \frac{z}{\langle z \mid e\rangle} \in \mathcal{V}_{e}
$$

Next choose a vector $d \in \mathcal{V}$ such that $\langle e \mid d\rangle=1$. Clearly, $\{e, d\}^{\perp}$ is $n$-dimensional. It will be convenient further on to choose coordinates $\left(z_{i}\right)_{i \in I_{n}}$ in $\{e, d\}^{\perp}$. Each $z \in \mathbb{C}^{n+2}$ can be written as

$$
z=\left[\begin{array}{c}
\left(z_{i}\right)_{i \in I_{n}} \\
z_{-m-1} \\
z_{m+1}
\end{array}\right]=\left(z_{i}\right)_{i \in I_{n}}+z_{-m-1} e+z_{m+1} d
$$

where $\left(z_{i}\right)_{i \in I_{n}} \in\{e, d\}^{\perp} \simeq \mathbb{C}^{n},\left(z_{-m-1}, z_{m+1}\right) \in \mathbb{C}^{2}$.

Using $y=\left(y_{i}\right)_{i \in I_{n}}$ as generic variables in $\mathbb{C}^{n}$, and noting that $z_{m+1}=\langle z \mid e\rangle$, we see that $\mathcal{Y}_{e}$ can be identified with $\mathbb{C}^{n}$ through the map

$$
\mathbb{C}^{n} \ni y \mapsto \mathbb{C}^{\times}\left[\begin{array}{c}
y \\
-\frac{\langle y \mid y\rangle}{2} \\
1
\end{array}\right] \in \mathcal{Y}_{e} .
$$

With this identification, the section (6.14) can be written as

$$
\mathbb{C}^{n} \ni y \mapsto\left[\begin{array}{c}
y \\
-\frac{\langle y \mid y\rangle}{2} \\
1
\end{array}\right] \in \mathcal{V}_{e}
$$


Remark 6.3. The above discussion shows that $\mathcal{Y}_{e}$ has a natural structure of the affine $n$-dimensional Euclidean space. The choice of $d \in \mathcal{V}_{e}$ (which does not influence the definition of $\mathcal{Y}_{e}$ ) determines the origin of coordinates in $\mathcal{Y}_{e}$.

The stabilizer of $\left[\begin{array}{l}0 \\ 1 \\ 0\end{array}\right]=e \in \mathcal{V}$ inside $\mathrm{O}\left(\mathbb{C}^{n+2}\right)$ is isomorphic to $\mathbb{C}^{n} \rtimes \mathrm{O}\left(\mathbb{C}^{n}\right)$, and is given by

$$
\left[\begin{array}{ccc}
\mathbb{1} & 0 & w \\
-w^{\#} & 1 & -\frac{1}{2}\langle w \mid w\rangle \\
0 & 0 & 1
\end{array}\right]\left[\begin{array}{lll}
\beta & 0 & 0 \\
0 & 1 & 0 \\
0 & 0 & 1
\end{array}\right], \quad \beta \in \mathrm{O}\left(\mathbb{C}^{n}\right), \quad w \in \mathbb{C}^{n}
$$

The stabilizer of $\mathbb{C}^{\times} e \in \mathcal{Y}$ inside $\mathrm{O}\left(\mathbb{C}^{n+2}\right)$ is isomorphic to $\mathbb{C}^{n} \rtimes\left(\mathrm{O}\left(\mathbb{C}^{n}\right) \times \mathrm{O}\left(\mathbb{C}^{2}\right)\right)$ and is given by

$$
\left[\begin{array}{ccc}
\mathbb{1} & 0 & w \\
-w^{\#} & 1 & -\frac{1}{2}\langle w \mid w\rangle \\
0 & 0 & 1
\end{array}\right]\left[\begin{array}{ccc}
\beta & 0 & 0 \\
0 & s & 0 \\
0 & 0 & s^{-1}
\end{array}\right], \quad \beta \in \mathrm{O}\left(\mathbb{C}^{n}\right), \quad w \in \mathbb{C}^{n}, \quad s \in \mathbb{C}^{\times} .
$$

\subsection{Laplacian on bundle of homogeneous functions}

Let $\mathcal{V}_{0}$ be an open subset of $\mathcal{V}$ and $\eta \in \mathbb{C}$. We define $\Lambda^{\eta}\left(\mathcal{V}_{0}\right)$ to be the set of holomorphic functions on $\mathcal{V}_{0}$ homogeneous of degree $\eta$. (See Sections 4.4 and 4.5 for a discussion.)

Clearly, $B \in \operatorname{so}\left(\mathbb{C}^{n+2}\right)$ preserves $\Lambda^{\eta}\left(\mathcal{V}_{0}\right)$. We will denote by $B^{\diamond, \eta}$ the restriction of $B \in \operatorname{hol}(\mathcal{V})$ to $\Lambda^{\eta}\left(\mathcal{V}_{0}\right)$.

Clearly, $\alpha \in \mathrm{O}\left(\mathbb{C}^{n+2}\right)$ maps $\Lambda^{\eta}\left(\mathcal{V}_{0}\right)$ onto $\Lambda^{\eta}\left(\alpha\left(\mathcal{V}_{0}\right)\right)$. We will denote by $\alpha^{\diamond, \eta}$ the restriction of $\alpha$ to $\Lambda^{\eta}\left(\mathcal{V}_{0}\right)$. Thus we have representations

$$
\operatorname{so}\left(\mathbb{C}^{n+2}\right) \ni B \mapsto B^{\diamond, \eta}, \quad \mathrm{O}\left(\mathbb{C}^{n+2}\right) \ni \alpha \mapsto \alpha^{\diamond, \eta} .
$$

We find the following theorem curious because it allows in some situations to restrict a second order differential operator to a submanifold.

Theorem 6.4. Let $\Omega \subset \mathbb{C}^{n+2}$ be open. Let $K \in \mathcal{A}(\Omega)$ be homogeneous of degree $\frac{2-n}{2}$ such that

$$
\left.K\right|_{c V \cap \Omega}=0
$$

Then

$$
\left.\Delta_{\mathbb{C}^{n+2}} K\right|_{\mathcal{V} \cap \Omega}=0
$$

Proof. We will give two proofs. Each of the proofs will provide us with a formula, which will be useful later on.

Method I. We use the decomposition $\mathbb{C}^{n+2}=\mathbb{C}^{n} \oplus \mathbb{C}^{2}$. As usual, we will denote by $\langle z \mid z\rangle$ the square of $z \in \mathbb{C}^{n+2}$, by $D_{\mathbb{C}^{n+2}}$ the generator of dilations, by $\mathcal{C}_{\mathbb{C}^{n+2}}$ the Casimir operator and by $\Delta_{\mathbb{C}^{n+2}}$ the Laplacian on $\mathbb{C}^{n+2}$. We will also need the corresponding objects on $\mathbb{C}^{n}:\langle z \mid z\rangle_{\mathbb{C}^{n}}$, $D_{\mathbb{C}^{n}}, \mathcal{C}_{\mathbb{C}^{n}}$, and $\Delta_{\mathbb{C}^{n}}$. We will write

$$
\begin{array}{ll}
\langle z \mid z\rangle_{m+1}:=2 z_{-m-1} z_{m+1}, & \Delta_{m+1}:=2 \partial_{z_{-m-1}} \partial_{z_{m+1}}, \\
D_{m+1}:=z_{-m-1} \partial_{z_{-m-1}}+z_{m+1} \partial_{z_{m+1}}, & N_{m+1}:=z_{-m-1} \partial_{z_{-m-1}}-z_{m+1} \partial_{z_{m+1}} .
\end{array}
$$

Thus we have

$$
\langle z \mid z\rangle=\langle z \mid z\rangle_{\mathbb{C}^{n}}+\langle z \mid z\rangle_{m+1}, \quad \Delta_{\mathbb{C}^{n+2}}=\Delta_{\mathbb{C}^{n}}+\Delta_{m+1}, \quad D_{\mathbb{C}^{n+2}}=D_{\mathbb{C}^{n}}+D_{m+1}
$$


The following identity is a consequence of (5.1):

$$
\begin{aligned}
\langle z \mid z\rangle_{\mathbb{C}^{n}} \Delta_{\mathbb{C}^{n+2}}= & \langle z \mid z\rangle_{\mathbb{C}^{n}} \Delta_{\mathbb{C}^{n}}+\left(\langle z \mid z\rangle-\langle z \mid z\rangle_{m+1}\right) \Delta_{m+1} \\
= & \mathcal{C}_{\mathbb{C}^{n}}+\left(D_{\mathbb{C}^{n}}-\frac{2-n}{2}\right)^{2}-\left(\frac{2-n}{2}\right)^{2}+\langle z \mid z\rangle \Delta_{m+1}-D_{m+1}^{2}+N_{m+1}^{2} \\
= & \langle z \mid z\rangle \Delta_{m+1}+\left(D_{\mathbb{C}^{n}}-\frac{2-n}{2}-D_{m+1}\right)\left(D_{\mathbb{C}^{n+2}}-\frac{2-n}{2}\right) \\
& -\left(\frac{2-n}{2}\right)^{2}+\mathcal{C}_{\mathbb{C}^{n}}+N_{m+1}^{2} .
\end{aligned}
$$

$\left(\frac{2-n}{2}\right)^{2}$ is a scalar. $\mathcal{C}_{\mathbb{C}^{n}}$ and $N_{m+1}^{2}$ are polynomials in elements of $\operatorname{so}\left(\mathbb{C}^{n+2}\right) . \mathcal{V}$ is tangent to so $\left(\mathbb{C}^{n+2}\right)$. Therefore, all operators in the last line of (6.16) can be restricted to $\mathcal{V}$. The operator $D_{\mathbb{C}^{n+2}}-\frac{2-n}{2}$ vanishes on functions in $\Lambda^{\frac{2-n}{2}}(\Omega)$. The operator $\langle z \mid z\rangle \Delta_{m+1}$ is zero when restricted to $\mathcal{V}$.

Method II. We write $\mathbb{C}^{n+2}=\mathbb{C}^{n+1} \oplus \mathbb{C}$ with the distinguished variable denoted by $t$. We assume that the square of $z \in \mathbb{C}^{n+2}$ is given by

$$
\langle z \mid z\rangle=\langle z \mid z\rangle_{\mathbb{C}^{n+1}}+t^{2}
$$

We will use various operators on $\mathbb{C}^{n+1}: D_{\mathbb{C}^{n+1}}, \mathcal{C}_{\mathbb{C}^{n+1}}$, and $\Delta_{\mathbb{C}^{n+1}}$. We have

$$
D_{\mathbb{C}^{n+2}}=D_{\mathbb{C}^{n+1}}+t \partial_{t}, \quad \Delta_{\mathbb{C}^{n+2}}=\Delta_{\mathbb{C}^{n+1}}+\partial_{t}^{2} .
$$

We have the following identity

$$
\begin{aligned}
\langle z \mid z\rangle_{\mathbb{C}^{n+1}} \Delta_{\mathbb{C}^{n+2}}= & \langle z \mid z\rangle_{\mathbb{C}^{n+1}} \Delta_{\mathbb{C}^{n+1}}+\langle z \mid z\rangle \partial_{t}^{2}-t^{2} \partial_{t}^{2} \\
= & \mathcal{C}_{\mathbb{C}^{n+1}}+\left(D_{\mathbb{C}^{n+1}}+\frac{n-1}{2}\right)^{2}-\left(\frac{n-1}{2}\right)^{2}+\langle z \mid z\rangle \partial_{t}^{2} \\
& -\left(t \partial_{t}-\frac{1}{2}\right)^{2}+\left(\frac{1}{2}\right)^{2} \\
= & \langle z \mid z\rangle \partial_{t}^{2}+\left(D_{\mathbb{C}^{n+1}}+\frac{n}{2}-t \partial_{t}\right)\left(D_{\mathbb{C}^{n+2}}-\frac{2-n}{2}\right) \\
& -\left(\frac{n-2}{2}\right) \frac{n}{2}+\mathcal{C}_{\mathbb{C}^{n+1}} .
\end{aligned}
$$

Then we argue similarly as in Method I.

Using Theorem 6.4 we can restrict the Laplacian to functions in $\Lambda^{\eta}\left(\mathcal{V}_{0}\right)$ for $\eta=\frac{2-n}{2}$. More precisely, we introduce the following definition.

Let $k \in \Lambda^{\frac{2-n}{2}}\left(\mathcal{V}_{0}\right)$. Let $\Omega$ be any open subset of $\mathbb{C}^{n+2}$ such that $\mathcal{V}_{0}=\Omega \cap \mathcal{V}$, let $K \in \mathcal{A}(\Omega)$ be homogeneous of degree $\frac{2-n}{2}$ and

$$
k=\left.K\right|_{\mathcal{V}_{0}} .
$$

(We can always find such $\Omega$ and $K$.) Note that $\Delta_{\mathbb{C}^{n+2}} K$ is homogeneous of degree $\frac{-2-n}{2}$. We set

$$
\Delta_{\mathbb{C}^{n+2}}^{\diamond} k:=\left.\Delta_{\mathbb{C}^{n+2}} K\right|_{\mathcal{V}_{0}}
$$

By Theorem 6.4, the above definition does not depend on the choice of $\Omega$ and $K$ and defines a map

$$
\Delta_{\mathbb{C}^{n+2}}^{\diamond}: \Lambda^{\frac{2-n}{2}}\left(\mathcal{V}_{0}\right) \rightarrow \Lambda^{\frac{-2-n}{2}}\left(\mathcal{V}_{0}\right)
$$


Remark 6.5. Let us explain the notation $\Delta_{\mathbb{C}^{n+2}}^{\diamond}$ for the reduced Laplacian. We do not put the degree of homogeneity $\eta=\frac{2-n}{2}$ as a superscript, because it is fixed by Theorem 6.4 , unlike in the case of the representations of $\mathrm{so}\left(\mathbb{C}^{n+2}\right)$ and $\mathrm{O}\left(\mathbb{C}^{n+2}\right)$. The subscript $\mathbb{C}^{n+2}$ is a little confusing, because $\Delta_{\mathbb{C}^{n+2}}^{\diamond}$ acts on functions of only $n+1$ variables, and after fixing a section on functions of $n$ variables. However, the initial operator is clearly $\Delta_{\mathbb{C}^{n+2}}$. Finally, the diamond $\diamond$ is a symbol that we have already used in the context of line bundles.

Restricting (6.4) and (6.5) to $\Lambda^{\frac{2-n}{2}}\left(\mathcal{V}_{0}\right)$ we obtain

$$
\begin{aligned}
& B^{\diamond, \frac{-2-n}{2}} \Delta_{\mathbb{C}^{n+2}}^{\diamond}=\Delta_{\mathbb{C}^{n+2}}^{\diamond} B^{\diamond, \frac{2-n}{2}}, \quad B \in \mathrm{so}\left(\mathbb{C}^{n+2}\right), \\
& \alpha^{\diamond, \frac{-2-n}{2}} \Delta_{\mathbb{C}^{n+2}}^{\diamond}=\Delta_{\mathbb{C}^{n+2}}^{\diamond} \alpha^{\diamond, \frac{2-n}{2}}, \quad \alpha \in \mathrm{O}\left(\mathbb{C}^{n+2}\right) .
\end{aligned}
$$

The following proposition is the consequence of the proof of Theorem 6.4.

\section{Proposition 6.6.}

(1) In the notation of Method I of the proof of Theorem 6.4, we have

$$
\langle z \mid z\rangle_{\mathbb{C}^{n}} \Delta_{\mathbb{C}^{n+2}}^{\diamond}=\left(\frac{n-2}{2}\right)^{2}+\mathcal{C}_{\mathbb{C}^{n}}+N_{m+1}^{2}
$$

(2) In the notation of Method II of the proof of Theorem 6.4, we have

$$
\langle z \mid z\rangle_{\mathbb{C}^{n+1}} \Delta_{\mathbb{C}^{n+2}}^{\diamond}=-\left(\frac{n-2}{2}\right) \frac{n}{2}+\mathcal{C}_{\mathbb{C}^{n+1}}
$$

Proof. (6.18) follows from (6.16). (6.19) follows from (6.17).

\subsection{Conformal invariance of Laplacian for a general section}

The operator $\Delta_{\mathbb{C}^{n+2}}^{\diamond}$ is quite abstract. In this subsection we describe how to make it more explicit.

Consider an open set $\mathcal{Y}_{0}$ in $\mathcal{Y}$. Let $\mathcal{V}_{0}:=\pi^{-1}\left(\mathcal{Y}_{0}\right)$ and $\eta \in \frac{1}{2} \mathbb{Z}$. Choose a section $\gamma$ of the line bundle $\mathcal{V} \rightarrow \mathcal{Y}$ based on $\mathcal{Y}_{0}$. As in (4.14) and (4.13), we can introduce $\psi^{\gamma, \eta}: \Lambda^{\eta}\left(\mathcal{V}_{0}\right) \rightarrow \mathcal{A}\left(\mathcal{Y}_{0}\right)$ and its left inverse $\phi^{\gamma, \eta}$. We set

$$
\begin{aligned}
& B^{\gamma, \eta}:=\psi^{\gamma, \eta} B^{\diamond, \eta} \phi^{\gamma, \eta}=\psi^{\gamma, \eta} B \phi^{\gamma, \eta}, \quad B \in \mathrm{so}\left(\mathbb{C}^{n+2}\right), \\
& \alpha^{\gamma, \eta}:=\psi^{\gamma, \eta} \alpha^{\diamond, \eta} \phi^{\gamma, \eta}=\psi^{\gamma, \eta} \alpha \phi^{\gamma, \eta}, \quad \alpha \in \mathrm{O}\left(\mathbb{C}^{n+2}\right) .
\end{aligned}
$$

As explained in Propositions 4.1, 4.2 and 4.3, we obtain a representation and a local representation

$$
\begin{aligned}
& \operatorname{so}\left(\mathbb{C}^{n+2}\right) \ni B \mapsto B^{\gamma, \eta} \in \mathcal{A} \rtimes \operatorname{hol}\left(\mathcal{Y}_{0}\right), \\
& \mathrm{O}\left(\mathbb{C}^{n+2}\right) \ni \alpha \mapsto \alpha^{\gamma, \eta} \underset{\text { loc }}{\in} \mathcal{A}^{\times} \rtimes \operatorname{Hol}\left(\mathcal{Y}_{0}\right) .
\end{aligned}
$$

We also define

$$
\Delta_{\mathbb{C}^{n+2}}^{\gamma}:=\psi^{\gamma, \frac{-2-n}{2}} \Delta_{\mathbb{C}^{n+2}}^{\diamond} \phi^{\gamma, \frac{2-n}{2}}=\psi^{\gamma, \frac{-2-n}{2}} \Delta_{\mathbb{C}^{n+2}} \phi^{\gamma, \frac{2-n}{2}} .
$$

We have the identities

$$
\begin{array}{ll}
B^{\gamma, \frac{-2-n}{2}} \Delta_{\mathbb{C}^{n+2}}^{\gamma}=\Delta_{\mathbb{C}^{n+2}}^{\gamma} B^{\gamma, \frac{2-n}{2}}, & B \in \mathrm{so}\left(\mathbb{C}^{n+2}\right), \\
\alpha^{\gamma, \frac{-2-n}{2}} \Delta_{\mathbb{C}^{n+2}}^{\gamma}=\Delta_{\mathbb{C}^{n+2}}^{\gamma} \alpha^{\gamma, \frac{2-n}{2}}, & \alpha \in \mathrm{O}\left(\mathbb{C}^{n+2}\right) .
\end{array}
$$


Thus we have shown that (infinitesimal) conformal transformations of the $n$-dimensional manifold $\mathcal{Y}_{0}$ lead to generalized (infinitesimal) symmetries of $\Delta_{\mathbb{C} n+2}^{\gamma}$. Even if (in a somewhat different form) this is a known fact, it seems that our derivation is new and of interest. In particular, it shows that a large class of second order $n$-dimensional operators together with their generalized symmetries directly come from the $(n+2)$-dimensional Laplacian with its true symmetries.

\subsection{Conformal invariance of Laplacian on $\mathbb{C}^{n}$}

Let us describe more closely the above construction in the case of the section (6.14). In this case, instead of $\gamma$ we will write "fl", for flat. We identify of $\mathcal{Y}_{e}$ with $\mathbb{C}^{n}$. We can restrict (6.8) to an action of $\operatorname{so}\left(\mathbb{C}^{n+2}\right)$ on $\mathcal{Y}_{e}$, and (6.9) to a local action of $\mathrm{O}\left(\mathbb{C}^{n+2}\right)$ on $\mathcal{Y}_{e}$. Using (4.2) and (4.10), we obtain

$$
\operatorname{so}\left(\mathbb{C}^{n+2}\right) \ni B \mapsto B^{\mathrm{fl}} \in \operatorname{hol}\left(\mathbb{C}^{n}\right), \quad \mathrm{O}\left(\mathbb{C}^{n+2}\right) \ni \alpha \mapsto \alpha^{\mathrm{fl}} \underset{\operatorname{loc}}{\in \operatorname{Hol}}\left(\mathbb{C}^{n}\right) .
$$

We introduce $\psi^{\mathrm{fl}, \eta}: \Lambda^{\eta}\left(\mathcal{V}_{e}\right) \rightarrow \mathcal{A}\left(\mathbb{C}^{n}\right)$ and its left inverse $\phi^{\mathrm{fl}, \eta}$. (6.20) and (6.21) can be rewritten as

$$
\operatorname{so}\left(\mathbb{C}^{n+2}\right) \ni B \mapsto B^{\mathrm{fl}, \eta} \in \mathcal{A} \rtimes \operatorname{hol}\left(\mathbb{C}^{n}\right), \quad \mathrm{O}\left(\mathbb{C}^{n+2}\right) \ni \alpha \mapsto \alpha^{\mathrm{fl}, \eta} \underset{\text { loc }}{\in} \mathcal{A}^{\times} \rtimes \operatorname{Hol}\left(\mathbb{C}^{n}\right) .
$$

The $(n+2)$-dimensional Laplacian reduced to the flat section is just the usual $n$-dimensional Laplacian:

$$
\Delta_{\mathbb{C}^{n+2}}^{\mathrm{fl}}=\Delta_{\mathbb{C}^{n}}
$$

The symmetries (6.22) and (6.23) become the generalized symmetries of the usual Laplacian:

$$
\begin{array}{ll}
B^{\mathrm{fl}, \frac{-2-n}{2}} \Delta_{\mathbb{C}^{n}}=\Delta_{\mathbb{C}^{n}} B^{\mathrm{fl}, \frac{2-n}{2}}, & B \in \mathrm{So}\left(\mathbb{C}^{n+2}\right), \\
\alpha^{\mathrm{fl}, \frac{-2-n}{2}} \Delta_{\mathbb{C}^{n}}=\Delta_{\mathbb{C}^{n}} \alpha^{\mathrm{fl}, \frac{2-n}{2}}, & \alpha \in \mathrm{O}\left(\mathbb{C}^{n+2}\right) .
\end{array}
$$

Thus $\mathbb{C}^{n+2}$ serves to describe in a simple way conformal symmetries of $\mathbb{C}^{n}$. When used in this fashion, the space $\mathbb{C}^{n+2}$ will be sometimes called the extended space.

Below we sum up information about conformal symmetries on the level of the extended space $\mathbb{C}^{n+2}$ and the space $\mathbb{C}^{n}$. We will use the split coordinates, that is, $z \in \mathbb{C}^{n+2}$ and $y \in \mathbb{C}^{n}$ have the square

$$
\langle z \mid z\rangle=\sum_{j \in I_{n+2}} z_{-j} z_{j}, \quad\langle y \mid y\rangle=\sum_{j \in I_{n}} y_{-j} y_{j} .
$$

As a rule, if a given operator does not depend on $\eta$, we will omit $\eta$.

Cartan algebra of so $\left(\mathbb{C}^{n+2}\right)$. Cartan operators of $\operatorname{so}\left(\mathbb{C}^{n}\right), i=1, \ldots, m$ :

$$
N_{i}=z_{-i} \partial_{z_{-i}}-z_{i} \partial_{z_{i}}, \quad N_{i}^{\mathrm{fl}}=y_{-i} \partial_{y_{-i}}-y_{i} \partial_{y_{i}} .
$$

Generator of dilations:

$$
N_{m+1}=z_{-m-1} \partial_{z_{-m-1}}-z_{m+1} \partial_{z_{m+1}}, \quad N_{m+1}^{\mathrm{fl}, \eta}=\sum_{i \in I_{n}} y_{i} \partial_{y_{i}}-\eta=D_{\mathbb{C}^{n}}-\eta .
$$

Root operators. Roots of so( $\left(\mathbb{C}^{n}\right),|i|<|j|, i, j \in I_{n}$ :

$$
B_{i, j}=z_{-i} \partial_{z_{j}}-z_{-j} \partial_{z_{i}}, \quad B_{i, j}^{\mathrm{fl}}=y_{-i} \partial_{y_{j}}-y_{-j} \partial_{y_{i}}
$$


Generators of translations, $j \in I_{n}$ :

$$
B_{-m-1, j}=z_{m+1} \partial_{z_{j}}-z_{-j} \partial_{z_{-m-1}}, \quad B_{-m-1, j}^{\mathrm{fl}}=\partial_{y_{j}}
$$

Generators of special conformal transformations, $j \in I_{n}$ :

$$
B_{m+1, j}=z_{-m-1} \partial_{z_{j}}-z_{-j} \partial_{z_{m+1}}, \quad B_{m+1, j}^{\mathrm{fl}, \eta}=-\frac{1}{2}\langle y \mid y\rangle \partial_{y_{j}}+y_{-j} \sum_{i \in I_{n}} y_{i} \partial_{y_{i}}-\eta y_{-j} .
$$

Weyl symmetries. We will write $K$ for a function on $\mathbb{C}^{n+2}$ and $f$ for a function on $\mathbb{C}^{n}$. Reflection:

$$
\begin{aligned}
\tau_{0} K\left(z_{0}, \ldots\right) & =K\left(-z_{0}, \ldots\right), \\
\tau_{0}^{\mathrm{fl}} f\left(y_{0}, \ldots\right) & =f\left(-y_{0}, \ldots\right) .
\end{aligned}
$$

Flips, $j=1, \ldots, m$ :

$$
\begin{aligned}
& \tau_{j} K\left(\ldots, z_{-j}, z_{j}, \ldots, z_{-m-1}, z_{m+1}\right)=K\left(\ldots, z_{j}, z_{-j}, \ldots, z_{-m-1}, z_{m+1}\right), \\
& \tau_{j}^{\mathrm{fl}} f\left(\ldots, y_{-j}, y_{j}, \ldots\right)=f\left(\ldots, y_{j}, y_{-j}, \ldots\right) .
\end{aligned}
$$

Inversion:

$$
\begin{aligned}
& \tau_{m+1} K\left(\ldots, z_{-m-1}, z_{m+1}\right)=K\left(\ldots, z_{m+1}, z_{-m-1}\right), \\
& \tau_{m+1}^{\mathrm{fl}, \eta} f(y)=\left(-\frac{\langle y \mid y\rangle}{2}\right)^{\eta} f\left(-\frac{2 y}{\langle y \mid y\rangle}\right) .
\end{aligned}
$$

Permutations, $\sigma \in S_{m}$ :

$$
\begin{aligned}
& \sigma K\left(\ldots, z_{-j}, z_{j}, \ldots, z_{-m-1}, z_{m+1}\right)=K\left(\ldots, z_{-\sigma_{j}}, z_{\sigma_{j}}, \ldots, z_{-m-1}, z_{m+1}\right), \\
& \sigma^{\mathrm{fl}} f\left(\ldots, y_{-j}, y_{j}, \ldots\right)=f\left(\ldots, y_{-\sigma_{j}}, y_{\sigma_{j}}, \ldots\right) .
\end{aligned}
$$

Special conformal transformations, $j=1, \ldots, m$ :

$$
\begin{aligned}
& \sigma_{(j, m+1)} K\left(z_{-1}, z_{1}, \ldots, z_{-j}, z_{j}, \ldots, z_{-m-1}, z_{m+1}\right) \\
& =K\left(z_{-1}, z_{1}, \ldots, z_{-m-1}, z_{m+1}, \ldots, z_{-j}, z_{j}\right), \\
& \sigma_{(j, m+1)}^{\mathrm{fl}, \eta} f\left(y_{-1}, y_{1}, \ldots, y_{-j}, y_{j}, \ldots\right)=y_{j}^{\eta} f\left(\frac{y_{-1}}{y_{j}}, \frac{y_{1}}{y_{j}}, \ldots,-\frac{\langle y \mid y\rangle}{2 y_{j}}, \frac{1}{y_{j}}, \ldots\right) \text {. }
\end{aligned}
$$

\section{Laplacian:}

$$
\Delta_{\mathbb{C}^{n+2}}=\sum_{i \in I_{n+2}} \partial_{z_{i}} \partial_{z_{-i}}, \quad \Delta_{\mathbb{C}^{n+2}}^{\mathrm{fl}}=\sum_{i \in I_{n}} \partial_{y_{i}} \partial_{y_{-i}}=\Delta_{\mathbb{C}^{n}}
$$

Computations. Let us describe how to derive these formulas in an easy way. Consider $\mathbb{C}^{n+1} \times \mathbb{C}^{\times}$(defined by $z_{m+1} \neq 0$ ), which is an open dense subset of $\mathbb{C}^{n+2}$. Clearly, $\mathcal{V}_{e}$ is contained in $\mathbb{C}^{n+1} \times \mathbb{C}^{\times}$.

We will write $\Lambda^{\eta}\left(\mathbb{C}^{n+1} \times \mathbb{C}^{\times}\right)$for the space of functions homogeneous of degree $\eta$ on $\mathbb{C}^{n+1} \times \mathbb{C}^{\times}$. Instead of using the maps $\phi^{\mathrm{fl}, \eta}$ and $\psi^{\mathrm{fl}, \eta}$, as in (4.14) and (4.13), we will prefer $\Phi^{\mathrm{fl}, \eta}: \mathcal{A}\left(\mathbb{C}^{n}\right) \rightarrow$ $\Lambda^{\eta}\left(\mathbb{C}^{n+1} \times \mathbb{C}^{\times}\right)$and $\Psi^{\mathrm{fl}, \eta}: \Lambda^{\eta}\left(\mathbb{C}^{n+1} \times \mathbb{C}^{\times}\right) \rightarrow \mathcal{A}\left(\mathbb{C}^{n}\right)$ defined below.

For $K \in \Lambda^{\eta}\left(\mathbb{C}^{n+1} \times \mathbb{C}^{\times}\right)$, we define $\Psi^{\mathrm{fl}, \eta} K \in \mathcal{A}\left(\mathbb{C}^{n}\right)$ by

$$
\left(\Psi^{\mathrm{fl}, \eta} K\right)(y)=K\left(y,-\frac{\langle y \mid y\rangle}{2}, 1\right), \quad y \in \mathbb{C}^{n} .
$$


Let $f \in \mathcal{A}\left(\mathbb{C}^{n}\right)$. Then there exists a unique function in $\Lambda^{\eta}\left(\mathbb{C}^{n+1} \times \mathbb{C}^{\times}\right)$that extends $f$ and does not depend on $z_{-m-1}$. It is given by

$$
\left(\Phi^{\mathrm{fl}, \eta} f\right)\left(\ldots, z_{m}, z_{-m-1}, z_{m+1}\right):=z_{m+1}^{\eta} f\left(\ldots, \frac{z_{m}}{z_{m+1}}\right) .
$$

$\Psi^{\mathrm{fl}, \eta}$ is a left inverse of $\Phi^{\mathrm{fl}, \eta}$ :

$$
\Psi^{\mathrm{fl}, \eta} \Phi^{\mathrm{fl}, \eta}=\mathrm{id} .
$$

Clearly,

$$
\left.\Phi^{\mathrm{fl}, \eta} f\right|_{\mathcal{V}_{e}}=\phi^{\mathrm{fl}, \eta} f, \quad \Psi^{\mathrm{fl}, \eta} K=\psi^{\mathrm{fl}, \eta}\left(\left.K\right|_{\mathcal{V}_{e}}\right) .
$$

Moreover, functions in $\Lambda^{\eta}\left(\mathbb{C}^{n+1} \times \mathbb{C}^{\times}\right)$restricted to $\mathcal{V}_{e}$ are in $\Lambda^{\eta}\left(\mathcal{V}_{e}\right)$. Therefore,

$$
\begin{aligned}
& B^{\mathrm{fl}, \eta}=\Psi^{\mathrm{fl}, \eta} B \Phi^{\mathrm{fl}, \eta}, \quad B \in \mathrm{so}\left(\mathbb{C}^{n+2}\right), \\
& \alpha^{\mathrm{fl}, \eta}=\Psi^{\mathrm{fl} l, \eta} \alpha \Phi^{\mathrm{fl}, \eta}, \quad \alpha \in \mathrm{O}\left(\mathbb{C}^{n+2}\right) \text {. }
\end{aligned}
$$

Note also that

$$
\Delta_{\mathbb{C}^{n+2}}^{\mathrm{fl}}=\Psi^{\mathrm{fl}, \eta} \Delta_{\mathbb{C}^{n+2}} \Phi^{\mathrm{fl}, \eta}=\Delta_{\mathbb{C}^{n}}
$$

In practice, the above idea can be implemented by the following change of coordinates on $\mathbb{C}^{n+2}$ :

$$
y_{i}:=\frac{z_{i}}{z_{m+1}}, \quad i \in I_{n}, \quad R:=\sum_{i \in I_{n+2}} z_{i} z_{-i}, \quad p:=z_{m+1} .
$$

The inverse transformation is

$$
z_{i}=p y_{i}, \quad i \in I_{n}, \quad z_{-m-1}=\frac{1}{2}\left(\frac{R}{p}-p \sum_{i \in I_{n}} y_{i} y_{-i}\right), \quad z_{m+1}=p .
$$

The derivatives are equal to

$$
\begin{aligned}
& \partial_{z_{i}}=z_{m+1}^{-1} \partial_{y_{i}}+2 z_{-i} \partial_{R}, \quad i \in I_{n}, \quad \partial_{z_{-m-1}}=2 z_{m+1} \partial_{R}, \\
& \partial_{z_{m+1}}=\partial_{p}-z_{m+1}^{-2} \sum_{i \in I_{n}} z_{i} \partial_{y_{i}}+2 z_{-m-1} \partial_{R} .
\end{aligned}
$$

Note that these coordinates are defined on $\mathbb{C}^{n+1} \times \mathbb{C}^{\times} . \mathcal{V}_{e}$ is given by the condition $R=0$. The section (6.14) (see also (6.15)) is given by $p=1$.

For a function $y \mapsto f(y)$ we have

$$
\left(\Phi^{\mathrm{fl}, \eta} f\right)(y, R, p)=p^{\eta} f(y) .
$$

For a function $(y, R, p) \mapsto K(y, R, p)$ we have

$$
\left(\Psi^{\mathrm{fl}, \eta} K\right)(y)=K(y, 0,1) .
$$

Note also that on $\Lambda^{\eta}\left(\mathbb{C}^{n+1} \times \mathbb{C}^{\times}\right)$we have

$$
p \partial_{p}=\eta
$$




\subsection{Dimension $n=1$}

Let us illustrate the constructions of this section by describing the projective quadric in the lowest dimensions, where everything is very explicit. We start with dimension $n=1$.

The 1-dimensional projective quadric is isomorphic to the Riemann sphere or, what is the same, the 1-dimensional projective complex space:

$$
\mathcal{Y}^{1} \simeq \mathbb{C} \cup\{\infty\}=P^{1} \mathbb{C} .
$$

Indeed, consider $\mathbb{C}^{3}$ with the scalar product

$$
\langle z \mid z\rangle=z_{0}^{2}+2 z_{-1} z_{+1} .
$$

We can cover $\mathcal{Y}^{1}$ with two maps:

$$
\mathbb{C} \ni s \mapsto \phi_{+}(s)=\left(s, 1,-\frac{1}{2} s^{2}\right) \mathbb{C}^{\times} \in \mathcal{Y}^{1}, \quad \mathbb{C} \ni s \mapsto \phi_{-}(s)=\left(s,-\frac{1}{2} s^{2}, 1\right) \mathbb{C}^{\times} \in \mathcal{Y}^{1} .
$$

The transition map is

$$
\phi_{+}^{-1} \phi_{-}(s)=-\frac{2}{s} .
$$

The Lie algebra so $\left(\mathbb{C}^{3}\right)$ is spanned by

$$
B_{0,1}, \quad B_{0,-1}, \quad N_{1},
$$

with the commutation relations

$$
\left[B_{0,1}, B_{0,-1}\right]=N_{1}, \quad\left[N_{1}, B_{0,1}\right]=B_{0,1}, \quad\left[N_{1}, B_{0,-1}\right]=-B_{0,-1} .
$$

The Casimir operator is

$$
\mathcal{C}=2 B_{0,1} B_{0,-1}-N_{1}^{2}-N_{1}=2 B_{0,-1} B_{0,1}-N_{1}^{2}+N_{1}
$$

\subsection{Dimension $n=2$}

The 2-dimensional projective quadric is isomorphic to the product of two Riemann spheres:

$$
\mathcal{Y}^{2} \simeq P^{1} \mathbb{C} \times P^{1} \mathbb{C} .
$$

Indeed, consider $\mathbb{C}^{4}$ with the scalar product

$$
\langle z \mid z\rangle=2 z_{-1} z_{+1}+2 z_{-2} z_{+2} .
$$

We can cover $\mathcal{Y}^{2}$ with four maps:

$$
\begin{aligned}
& \mathbb{C} \times \mathbb{C} \ni(t, s) \mapsto \phi_{+1}(t, s)=(-t s, 1, t, s) \mathbb{C}^{\times} \in \mathcal{Y}^{2}, \\
& \mathbb{C} \times \mathbb{C} \ni(t, s) \mapsto \phi_{-1}(t, s)=(1,-t s, s, t) \mathbb{C}^{\times} \in \mathcal{Y}^{2}, \\
& \mathbb{C} \times \mathbb{C} \ni(t, s) \mapsto \phi_{+2}(t, s)=(-s,-t, 1, t s) \mathbb{C}^{\times} \in \mathcal{Y}^{2}, \\
& \mathbb{C} \times \mathbb{C} \ni(t, s) \mapsto \phi_{-2}(t, s)=(-t,-s,-t s, 1) \mathbb{C}^{\times} \in \mathcal{Y}^{2}
\end{aligned}
$$

Here are the transition maps:

$$
\begin{aligned}
& \phi_{-1}^{-1} \phi_{+1}(t, s)=\phi_{-2}^{-1} \phi_{+2}(t, s)=\left(-t^{-1},-s^{-1}\right), \\
& \phi_{-2}^{-1} \phi_{-1}(t, s)=\phi_{+2}^{-1} \phi_{+1}(t, s)=\left(-t^{-1}, s\right), \\
& \phi_{-2}^{-1} \phi_{+1}(t, s)=\phi_{+2}^{-1} \phi_{-1}(t, s)=\left(t,-s^{-1}\right) .
\end{aligned}
$$


The Lie algebra so $\left(\mathbb{C}^{4}\right)$ is spanned by

$$
N_{1}, \quad N_{2}, \quad B_{1,2}, \quad B_{1,-2}, \quad B_{-1,2}, \quad B_{-1,-2} .
$$

Its Casimir operator is

$$
\mathcal{C}=2 B_{1,2} B_{-1,-2}+2 B_{1,-2} B_{-1,2}-N_{1}^{2}-N_{2}^{2}+2 N_{1} .
$$

As is well known, so $\left(\mathbb{C}^{4}\right)$ decomposes into the direct sum $\mathrm{sO}_{+}\left(\mathbb{C}^{3}\right) \oplus \mathrm{sO}_{-}\left(\mathbb{C}^{3}\right)$ of two commuting Lie algebras isomorphic to so $\left(\mathbb{C}^{3}\right)$ spanned by

$$
B_{1,2}, \quad B_{-1,-2}, \quad N_{1}+N_{2} \quad \text { and } \quad B_{1,-2}, \quad B_{-1,2}, \quad N_{1}-N_{2}
$$

with the commutation relations

$$
\begin{array}{ll}
{\left[B_{1,2}, B_{-1,-2}\right]=N_{1}+N_{2},} & {\left[B_{1,-2}, B_{-1,2}\right]=N_{1}-N_{2},} \\
{\left[N_{1}+N_{2}, B_{1,2}\right]=2 B_{1,2},} & {\left[N_{1}-N_{2}, B_{1,-2}\right]=2 B_{1,-2},} \\
{\left[N_{1}+N_{2}, B_{-1,-2}\right]=-2 B_{-1,-2},} & {\left[N_{1}-N_{2}, B_{-1,2}\right]=-2 B_{-1,2} .}
\end{array}
$$

The corresponding Casimir operators are

$$
\begin{aligned}
& \mathcal{C}_{+}=2 B_{1,2} B_{-1,-2}-\frac{1}{2}\left(N_{1}+N_{2}\right)^{2}-N_{1}-N_{2}=2 B_{-1,-2} B_{1,2}-\frac{1}{2}\left(N_{1}+N_{2}\right)^{2}+N_{1}+N_{2}, \\
& \mathcal{C}_{-}=2 B_{1,-2} B_{-1,2}-\frac{1}{2}\left(N_{1}-N_{2}\right)^{2}-N_{1}+N_{2}=2 B_{-1,2} B_{1,-2}-\frac{1}{2}\left(N_{1}-N_{2}\right)^{2}+N_{1}-N_{2} .
\end{aligned}
$$

Thus

$$
\mathcal{C}=\mathcal{C}_{+}+\mathcal{C}_{-}
$$

In the enveloping algebra of $\operatorname{so}\left(\mathbb{C}^{4}\right)$ the operators $\mathcal{C}_{+}$and $\mathcal{C}_{-}$are distinct. They satisfy $\alpha\left(\mathcal{C}_{-}\right)=\mathcal{C}_{+}$for $\alpha \in \mathrm{O}\left(\mathbb{C}^{4}\right) \backslash \mathrm{SO}\left(\mathbb{C}^{4}\right)$, for instance for $\tau_{i}, i=1,2$.

However, inside the associative algebra of differential operators on $\mathbb{C}^{4}$ we have the identity

$$
B_{1,2} B_{-1,-2}-B_{1,-2} B_{-1,2}=N_{1} N_{2}+N_{2},
$$

which implies

$$
\mathcal{C}_{+}=\mathcal{C}_{-}
$$

inside this algebra. Therefore, represented in the algebra of differential operators we have

$$
\begin{aligned}
\mathcal{C} & =4 B_{1,2} B_{-1,-2}-\left(N_{1}+N_{2}\right)^{2}-2 N_{1}-2 N_{2} \\
& =4 B_{-1,-2} B_{1,2}-\left(N_{1}+N_{2}\right)^{2}+2 N_{1}+2 N_{2} \\
& =4 B_{1,-2} B_{-1,2}-\left(N_{1}-N_{2}\right)^{2}-2 N_{1}+2 N_{2} \\
& =4 B_{-1,2} B_{1,-2}-\left(N_{1}-N_{2}\right)^{2}+2 N_{1}-2 N_{2} .
\end{aligned}
$$

\section{$\begin{array}{ll}7 & \text { so }\left(\mathbb{C}^{6}\right) \text { and the hypergeometric equation }\end{array}$}

In this section we derive the hypergeometric operator and its $\operatorname{so}\left(\mathbb{C}^{6}\right)$ symmetries. We will consider the following levels:

(1) extended space $\mathbb{C}^{6}$ and the Laplacian $\Delta_{\mathbb{C}^{6}}$, 
(2) reduction to the so-called spherical section and the corresponding Laplace-Beltrami operator,

(3) depending on the choice of coordinates, separation of variables leads to the balanced or standard hypergeometric operator.

Alternatively, one can use a different derivation:

$(2)^{\prime}$ reduction to $\mathbb{C}^{4}$ and $\Delta_{\mathbb{C}^{4}}$ with help of the flat section,

$(3)^{\prime}$ with appropriate coordinates, separation of variables leads to the balanced or standard hypergeometric operator.

A separate subsection is devoted to factorizations of the hypergeometric operator. We will see that they are closely related to so $\left(\mathbb{C}^{4}\right)$ subalgebras of so $\left(\mathbb{C}^{6}\right)$ and their Casimir operators.

\subsection{Extended space $\mathbb{C}^{6}$}

We consider $\mathbb{C}^{6}$ with the coordinates

$z_{-1}, \quad z_{1}, \quad z_{-2}, \quad z_{2}, \quad z_{-3}, \quad z_{3}$

and the scalar product given by

$$
\langle z \mid z\rangle=2 z_{-1} z_{1}+2 z_{-2} z_{2}+2 z_{-3} z_{3} .
$$

Lie algebra $\mathrm{so}\left(\mathbb{C}^{6}\right)$. Cartan algebra:

$$
N_{i}=z_{-i} \partial_{z_{-i}}-z_{i} \partial_{z_{i}}, \quad i=1,2,3 .
$$

Root operators:

$$
B_{i, j}=z_{-i} \partial_{j}-z_{-j} \partial_{i}, \quad 1 \leq|i|<|j| \leq 3 .
$$

Weyl symmetries. Transpositions:

$$
\begin{aligned}
& \sigma_{(12)} K\left(z_{-1}, z_{1}, z_{-2}, z_{2}, z_{-3}, z_{3}\right)=K\left(z_{-2}, z_{2}, z_{-1}, z_{1}, z_{-3}, z_{3}\right), \\
& \sigma_{(13)} K\left(z_{-1}, z_{1}, z_{-2}, z_{2}, z_{-3}, z_{3}\right)=K\left(z_{-3}, z_{3}, z_{-2}, z_{2}, z_{-1}, z_{1}\right), \\
& \sigma_{(23)} K\left(z_{-1}, z_{1}, z_{-2}, z_{2}, z_{-3}, z_{3}\right)=K\left(z_{-1}, z_{1}, z_{-3}, z_{3}, z_{-2}, z_{2}\right) .
\end{aligned}
$$

Cycles:

$$
\begin{aligned}
& \sigma_{(123)} K\left(z_{-1}, z_{1}, z_{-2}, z_{2}, z_{-3}, z_{3}\right)=K\left(z_{-3}, z_{3}, z_{-1}, z_{1}, z_{-2}, z_{2}\right), \\
& \sigma_{(132)} K\left(z_{-1}, z_{1}, z_{-2}, z_{2}, z_{-3}, z_{3}\right)=K\left(z_{-2}, z_{2}, z_{-3}, z_{3}, z_{-1}, z_{1}\right) .
\end{aligned}
$$

Flips:

$$
\begin{aligned}
& \tau_{1} K\left(z_{-1}, z_{1}, z_{-2}, z_{2}, z_{-3}, z_{3}\right)=K\left(z_{1}, z_{-1}, z_{-2}, z_{2}, z_{-3}, z_{3}\right), \\
& \tau_{2} K\left(z_{-1}, z_{1}, z_{-2}, z_{2}, z_{-3}, z_{3}\right)=K\left(z_{-1}, z_{1}, z_{2}, z_{-2}, z_{-3}, z_{3}\right), \\
& \tau_{3} K\left(z_{-1}, z_{1}, z_{-2}, z_{2}, z_{-3}, z_{3}\right)=K\left(z_{-1}, z_{1}, z_{-2}, z_{2}, z_{3}, z_{-3}\right) .
\end{aligned}
$$

\section{Laplacian:}

$$
\Delta_{\mathbb{C}^{6}}=2 \partial_{z_{-1}} \partial_{z_{1}}+2 \partial_{z_{-2}} \partial_{z_{2}}+2 \partial_{z_{-3}} \partial_{z_{3}} .
$$

\section{Symmetries:}

$$
\begin{array}{ll}
N_{i} \Delta_{\mathbb{C}^{6}}=\Delta_{\mathbb{C}^{6}} N_{i}, & 1 \leq i \leq 3, \\
B_{i, j} \Delta_{\mathbb{C}^{6}}=\Delta_{\mathbb{C}^{6}} B_{i, j}, & 1 \leq|i|<|j| \leq 3, \\
\sigma \Delta_{\mathbb{C}^{6}}=\Delta_{\mathbb{C}^{6}} \sigma, & \sigma \in S_{3}, \\
\tau_{j} \Delta_{\mathbb{C}^{6}}=\Delta_{\mathbb{C}^{6}} \tau_{j}, & 1 \leq j \leq 3 .
\end{array}
$$




\subsection{Spherical section}

In this subsection we consider the section of the quadric

$$
\mathcal{V}^{5}:=\left\{z \in \mathbb{C}^{6}: 2 z_{-1} z_{1}+2 z_{-2} z_{2}+2 z_{-3} z_{3}=0\right\}
$$

given by equations

$$
4=2\left(z_{-1} z_{1}+z_{-2} z_{2}\right)=-2 z_{3} z_{-3} .
$$

We will call it the spherical section, because it coincides with $\mathcal{S}^{3}(4) \times \mathcal{S}^{1}(-4)$. The superscript used for this section will be "sph" for spherical.

We will see that this section is well suited to obtain the hypergeometric equation, both in the balanced and standard form, because its conformal factor is trivially equal to 1.

As a preparation for a discussion of this section, let us choose the coordinates

$$
\begin{aligned}
& r=\sqrt{2\left(z_{-1} z_{1}+z_{-2} z_{2}\right)}, \quad p=\sqrt{2 z_{3} z_{-3}}, \quad w=\frac{z_{-1} z_{1}}{z_{-1} z_{1}+z_{-2} z_{2}}, \\
& u_{1}=\sqrt{\frac{z_{-1}}{z_{1}}}, \quad u_{2}=\sqrt{\frac{z_{-2}}{z_{2}}}, \quad u_{3}=\sqrt{\frac{z_{-3}}{z_{3}}} .
\end{aligned}
$$

The null quadric in these coordinates is given by $r^{2}+p^{2}=0$. The generator of dilations is

$$
D_{\mathbb{C}^{6}}=r \partial_{r}+p \partial_{p}
$$

The spherical section is given by the condition $r^{2}=4$.

Let us now describe in detail various objects in the spherical section.

Lie algebra $\mathrm{so}\left(\mathbb{C}^{6}\right)$. Cartan operators:

$$
N_{1}^{\mathrm{sph}}=u_{1} \partial_{u_{1}}, \quad N_{2}^{\mathrm{sph}}=u_{2} \partial_{u_{2}}, \quad N_{3}^{\mathrm{sph}}=u_{3} \partial_{u_{3}} .
$$

Roots:

$$
\begin{aligned}
& B_{2,1}^{\mathrm{sph}}=u_{1} u_{2} \sqrt{w(1-w)}\left(\partial_{w}-\frac{N_{1}^{\mathrm{sph}}}{2 w}+\frac{N_{2}^{\mathrm{sph}}}{2(1-w)}\right), \\
& B_{-2,-1}^{\mathrm{sph}}=\frac{1}{u_{1} u_{2}} \sqrt{w(1-w)}\left(\partial_{w}+\frac{N_{1}^{\mathrm{sph}}}{2 w}-\frac{N_{2}^{\mathrm{sph}}}{2(1-w)}\right), \\
& B_{2,-1}^{\mathrm{sph}}=\frac{u_{2}}{u_{1}} \sqrt{w(1-w)}\left(\partial_{w}+\frac{N_{1}^{\mathrm{sph}}}{2 w}+\frac{N_{2}^{\mathrm{sph}}}{2(1-w)}\right), \\
& B_{-2,1}^{\mathrm{sph}}=\frac{u_{1}}{u_{2}} \sqrt{w(1-w)}\left(\partial_{w}-\frac{N_{1}^{\mathrm{sph}}}{2 w}-\frac{N_{2}^{\mathrm{sph}}}{2(1-w)}\right), \\
& B_{3,1}^{\mathrm{sph}, \eta}=\frac{\mathrm{i}}{2} u_{1} u_{3} \sqrt{w}\left(\eta+2(1-w) \partial_{w}-\frac{N_{1}^{\mathrm{sph}}}{w}-N_{3}^{\mathrm{sph}}\right), \\
& B_{-3,-1}^{\mathrm{sph},}=\frac{\mathrm{i}}{2} \frac{1}{u_{1} u_{3}} \sqrt{w}\left(\eta+2(1-w) \partial_{w}+\frac{N_{1}^{\mathrm{sph}}}{w}+N_{3}^{\mathrm{sph}}\right), \\
& B_{3,-1}^{\mathrm{sph}, \eta}=\frac{\mathrm{i}}{2} \frac{u_{3}}{u_{1}} \sqrt{w}\left(\eta+2(1-w) \partial_{w}+\frac{\left.N_{1}^{\mathrm{sph}}-N_{3}^{\mathrm{sph}}\right)}{w},\right.
\end{aligned}
$$




$$
\begin{aligned}
& B_{-3,1}^{\mathrm{sph}, \eta}=\frac{\mathrm{i}}{2} \frac{u_{1}}{u_{3}} \sqrt{w}\left(\eta+2(1-w) \partial_{w}-\frac{N_{1}^{\mathrm{sph}}}{w}+N_{3}^{\mathrm{sph}}\right), \\
& B_{3,2}^{\mathrm{sph}, \eta}=\frac{\mathrm{i}}{2} u_{2} u_{3} \sqrt{1-w}\left(\eta-2 w \partial_{w}-\frac{N_{2}^{\mathrm{sph}}}{1-w}-N_{3}^{\mathrm{sph}}\right), \\
& B_{-3,-2}^{\mathrm{sph}, \eta}=\frac{\mathrm{i}}{2} \frac{1}{u_{2} u_{3}} \sqrt{1-w}\left(\eta-2 w \partial_{w}+\frac{N_{2}^{\mathrm{sph}}}{1-w}+N_{3}^{\mathrm{sph}}\right), \\
& B_{3,-2}^{\mathrm{sph}, \eta}=\frac{\mathrm{i}}{2} \frac{u_{3}}{u_{2}} \sqrt{1-w}\left(\eta-2 w \partial_{w}+\frac{N_{2}^{\mathrm{sph}}}{1-w}-N_{3}^{\mathrm{sph}}\right), \\
& B_{-3,2}^{\mathrm{sph}, \eta}=\frac{\mathrm{i}}{2} \frac{u_{2}}{u_{3}} \sqrt{1-w}\left(\eta-2 w \partial_{w}-\frac{N_{2}^{\mathrm{sph}}}{1-w}+N_{3}^{\mathrm{sph}}\right) .
\end{aligned}
$$

Weyl symmetries. Transpositions:

$$
\begin{aligned}
& \sigma_{12}^{\mathrm{sph}, \eta} f\left(w, u_{1}, u_{2}, u_{3}\right)=f\left(1-w, u_{2}, u_{1}, u_{3}\right), \\
& \sigma_{13}^{\mathrm{sph}, \eta} f\left(w, u_{1}, u_{2}, u_{3}\right)=(\mathrm{i} \sqrt{w})^{\eta} f\left(\frac{1}{w}, u_{3}, u_{2}, u_{1}\right), \\
& \sigma_{23}^{\mathrm{sph}, \eta} f\left(w, u_{1}, u_{2}, u_{3}\right)=(\mathrm{i} \sqrt{1-w})^{\eta} f\left(\frac{w}{w-1}, u_{1}, u_{3}, u_{2}\right) .
\end{aligned}
$$

Cycles:

$$
\begin{aligned}
& \sigma_{312}^{\mathrm{sph}, \eta} f\left(w, u_{1}, u_{2}, u_{3}\right)=(\mathrm{i} \sqrt{1-w})^{\eta} f\left(\frac{1}{1-w}, u_{3}, u_{1}, u_{2}\right), \\
& \sigma_{231}^{\mathrm{sph}, \eta} f\left(w, u_{1}, u_{2}, u_{3}\right)=(\mathrm{i} \sqrt{w})^{\eta} f\left(1-\frac{1}{w}, u_{2}, u_{3}, u_{1}\right) .
\end{aligned}
$$

Flips:

$$
\begin{aligned}
\tau_{1}^{\mathrm{sph}, \eta} f\left(w, u_{1}, u_{2}, u_{3}\right) & =f\left(w, \frac{1}{u_{1}}, u_{2}, u_{3}\right), \\
\tau_{2}^{\mathrm{sph}, \eta} f\left(w, u_{1}, u_{2}, u_{3}\right) & =f\left(w, u_{1}, \frac{1}{u_{2}}, u_{3}\right), \\
\tau_{3}^{\mathrm{sph}, \eta} f\left(w, u_{1}, u_{2}, u_{3}\right) & =f\left(w, u_{1}, u_{2}, \frac{1}{u_{3}}\right) .
\end{aligned}
$$

The Laplacian in coordinates $(7.5)$ is

$$
\begin{aligned}
\Delta_{\mathbb{C}^{6}}=\frac{4}{r^{2}}( & \frac{1}{4}\left(\left(r \partial_{r}\right)^{2}+2\left(r \partial_{r}\right)+\frac{r^{2}}{p^{2}}\left(p \partial_{p}\right)^{2}\right) \\
& \left.+\partial_{w} w(1-w) \partial_{w}-\frac{\left(u_{1} \partial_{u_{1}}\right)^{2}}{4 w}-\frac{\left(u_{1} \partial_{u_{2}}\right)^{2}}{4(1-w)}-\frac{r^{2}}{p^{2}} \frac{\left(u_{3} \partial_{u_{3}}\right)^{2}}{4}\right) .
\end{aligned}
$$

Using

$$
\begin{aligned}
\left(r \partial_{r}\right)^{2}+2 r \partial_{r}+\frac{r^{2}}{p^{2}}\left(p \partial_{p}\right)^{2}= & \left(r \partial_{r}+\frac{r^{2}}{p^{2}}\left(p \partial_{p}\right)+1\right)\left(r \partial_{r}+p \partial_{p}+1\right)-1 \\
& -\left(\frac{r^{2}}{p^{2}}+1\right)\left(p \partial_{p}\right)\left(r \partial_{r}+1\right)
\end{aligned}
$$


$r^{2}+p^{2}=0$ and $r \partial_{r}+p \partial_{p}=-1$, we obtain

$$
\Delta_{\mathbb{C}^{6}}^{\diamond}=\frac{4}{r^{2}}\left(\partial_{w} w(1-w) \partial_{w}-\frac{\left(u_{1} \partial_{u_{1}}\right)^{2}}{4 w}-\frac{\left(u_{2} \partial_{u_{2}}\right)^{2}}{4(1-w)}+\frac{\left(u_{3} \partial_{u_{3}}\right)^{2}}{4}-\frac{1}{4}\right) .
$$

To convert $\Delta_{\mathbb{C}^{6}}^{\diamond}$ into the reduced Laplacian $\Delta_{\mathbb{C}^{6}}^{\text {sph }}$, we simply remove the prefactor $\frac{4}{r^{2}}$, obtaining the Laplace-Beltrami operator on $\mathcal{S}^{3}(4) \times \mathcal{S}^{1}(-4)$ :

$$
\Delta_{\mathbb{C}^{6}}^{\mathrm{sph}}=\partial_{w} w(1-w) \partial_{w}-\frac{\left(N_{1}^{\mathrm{sph}}\right)^{2}}{4 w}-\frac{\left(N_{2}^{\mathrm{sph}}\right)^{2}}{4(1-w)}+\frac{\left(N_{3}^{\mathrm{sph}}\right)^{2}}{4}-\frac{1}{4} .
$$

\section{Generalized symmetries:}

$$
\begin{array}{ll}
N_{i}^{\mathrm{sph}} \Delta_{\mathbb{C}^{6}}^{\mathrm{sph}}=\Delta_{\mathbb{C}^{6}}^{\mathrm{sph}} N_{i}^{\mathrm{sph}}, & 1 \leq i \leq 3, \\
B_{i, j}^{\mathrm{sph},-3} \Delta_{\mathbb{C}^{6}}^{\mathrm{sph}}=\Delta_{\mathbb{C}^{6}}^{\mathrm{sph}} B_{i, j}^{\mathrm{sph},-1}, & 1 \leq|i|<|j| \leq 3, \\
\sigma^{\mathrm{sph},-3} \Delta_{\mathbb{C}^{6}}^{\mathrm{sph}}=\Delta_{\mathbb{C}^{6}}^{\mathrm{sph}} \sigma^{\mathrm{sph},-1}, & \sigma \in S_{3}, \\
\tau_{j}^{\mathrm{sph}} \Delta_{\mathbb{C}^{6}}^{\mathrm{sph}}=\Delta_{\mathbb{C}^{6}}^{\mathrm{sph}} \tau_{j}^{\mathrm{sph}}, & 1 \leq j \leq 3 .
\end{array}
$$

\subsection{Balanced hypergeometric operator}

Using the spherical section we make an ansatz

$$
f\left(w, u_{1}, u_{2}, u_{3}\right)=u_{1}^{\alpha} u_{2}^{\beta} u_{3}^{\mu} F(w) .
$$

Clearly,

$$
N_{1}^{\mathrm{sph}} f=\alpha f, \quad N_{2}^{\mathrm{sph}} f=\beta f, \quad N_{3}^{\mathrm{sph}} f=\mu f .
$$

Therefore, on functions of the form $(7.11), \Delta_{\mathbb{C}^{6}}^{\mathrm{sph}}$, that is $(7.6)$, coincides with the balanced hypergeometric operator (2.2). The generalized symmetries for the roots (7.8), for the permutations (7.9) and for the flips (7.10) coincide with the transmutation relations, with the discrete symmetries, and with the sign changes of $\alpha, \beta, \mu$ of the balanced hypergeometric equation, respectively; see Section 2.1.

\subsection{Standard hypergeometric operator}

Alternatively, we can slightly change the coordinates (7.5), replacing $u_{1}$, $u_{2}$ with

$$
\tilde{u}_{1}:=\frac{z_{-1}}{\sqrt{z_{-1} z_{1}+z_{-2} z_{2}}}=u_{1} \sqrt{w}, \quad \tilde{u}_{2}:=\frac{z_{-2}}{\sqrt{z_{-1} z_{1}+z_{-2} z_{2}}}=u_{2} \sqrt{1-w}
$$

As compared with the previous coordinates, we need to replace $\partial_{w}$ with

$$
\partial_{w}+N_{1}^{\mathrm{sph}} \frac{1}{2 w}+N_{2}^{\mathrm{sph}} \frac{1}{2(w-1)} .
$$

Let us only quote the results for the Cartan operators

$$
N_{1}^{\mathrm{sph}}=\tilde{u}_{1} \partial_{\tilde{u}_{1}}, \quad N_{2}^{\mathrm{sph}}=\tilde{u}_{2} \partial_{\tilde{u}_{2}}, \quad N_{3}^{\mathrm{sph}}=u_{3} \partial_{u_{3}},
$$

and the reduced Laplacian:

$$
\begin{aligned}
\Delta_{\mathbb{C}^{6}}^{\mathrm{sph}}= & w(1-w) \partial_{w}^{2}+\left(\left(1+N_{1}^{\mathrm{sph}}\right)(1-w)-\left(1+N_{2}^{\mathrm{sph}}\right) w\right) \partial_{w} \\
& +\frac{1}{4}\left(N_{3}^{\mathrm{sph}}\right)^{2}-\frac{1}{4}\left(N_{1}^{\mathrm{sph}}+N_{2}^{\mathrm{sph}}+1\right)^{2} .
\end{aligned}
$$


If we now make the ansatz

$$
f\left(w, \tilde{u}_{1}, \tilde{u}_{2}, u_{3}\right)=\tilde{u}_{1}^{\alpha} \tilde{u}_{2}^{\beta} u_{3}^{\mu} \tilde{F}(w),
$$

then clearly,

$$
N_{1}^{\mathrm{sph}} f=\alpha f, \quad N_{2}^{\mathrm{sph}} f=\beta f, \quad N_{3}^{\mathrm{sph}} f=\mu f .
$$

It is easy to see that on functions of the form $(7.13), \Delta_{\mathbb{C}^{6}}^{\mathrm{sph}}$ coincides with the standard hypergeometric operator (2.1). When (7.12) is applied to root operators and Weyl symmetries, we also obtain the symmetries of the standard hypergeometric operator described in [6].

\subsection{Factorizations}

In the Lie algebra so $\left(\mathbb{C}^{6}\right)$ represented in $(7.1)$ we have 3 distinguished Lie subalgebras isomorphic to $\mathrm{so}\left(\mathbb{C}^{4}\right)$ : in an obvious notation,

$$
\operatorname{SO}_{12}\left(\mathbb{C}^{4}\right), \quad \operatorname{SO}_{13}\left(\mathbb{C}^{4}\right), \quad \operatorname{SO}_{23}\left(\mathbb{C}^{4}\right) .
$$

By (6.25), the corresponding Casimir operators are

$$
\begin{aligned}
\mathcal{C}_{12} & =4 B_{1,2} B_{-1,-2}-\left(N_{1}+N_{2}\right)^{2}-2 N_{1}-2 N_{2}=4 B_{-1,-2} B_{1,2}-\left(N_{1}+N_{2}\right)^{2}+2 N_{1}+2 N_{2} \\
& =4 B_{1,-2} B_{-1,2}-\left(N_{1}-N_{2}\right)^{2}-2 N_{1}+2 N_{2}=4 B_{-1,2} B_{1,-2}-\left(N_{1}-N_{2}\right)^{2}+2 N_{1}-2 N_{2}, \\
\mathcal{C}_{13} & =4 B_{1,3} B_{-1,-3}-\left(N_{1}+N_{3}\right)^{2}-2 N_{1}-2 N_{3}=4 B_{-1,-3} B_{1,3}-\left(N_{1}+N_{3}\right)^{2}+2 N_{1}+2 N_{3} \\
& =4 B_{1,-3} B_{-1,3}-\left(N_{1}-N_{2}\right)^{2}-2 N_{1}+2 N_{3}=4 B_{-1,3} B_{1,-3}-\left(N_{1}-N_{3}\right)^{2}+2 N_{1}-2 N_{3}, \\
\mathcal{C}_{23} & =4 B_{2,3} B_{-2,-3}-\left(N_{2}+N_{3}\right)^{2}-2 N_{2}-2 N_{3}=4 B_{-2,-3} B_{2,3}-\left(N_{2}+N_{3}\right)^{2}+2 N_{2}+2 N_{3} \\
& =4 B_{2,-3} B_{-2,3}-\left(N_{2}-N_{2}\right)^{2}-2 N_{2}+2 N_{3}=4 B_{-2,3} B_{2,-3}-\left(N_{2}-N_{3}\right)^{2}+2 N_{2}-2 N_{3} .
\end{aligned}
$$

After the reduction described in (6.18), we obtain the identities

$$
\begin{aligned}
& \left(2 z_{-1} z_{1}+2 z_{-2} z_{2}\right) \Delta_{\mathbb{C}^{6}}^{\diamond}=-1+\mathcal{C}_{12}^{\diamond,-1}+\left(N_{3}^{\diamond,-1}\right)^{2}, \\
& \left(2 z_{-1} z_{1}+2 z_{-3} z_{3}\right) \Delta_{\mathbb{C}^{6}}^{\diamond}=-1+\mathcal{C}_{13}^{\diamond,-1}+\left(N_{2}^{\diamond,-1}\right)^{2}, \\
& \left(2 z_{-2} z_{2}+2 z_{-3} z_{3}\right) \Delta_{\mathbb{C}^{6}}^{\diamond}=-1+\mathcal{C}_{23}^{\diamond,-1}+\left(N_{1}^{\diamond,-1}\right)^{2} .
\end{aligned}
$$

If we use the spherical section, (7.14a), (7.14b), (7.14c) become

$$
\begin{aligned}
& 4 \Delta_{\mathbb{C}^{6}}^{\mathrm{sph}}=-1+\mathcal{C}_{12}^{\mathrm{sph},-1}+\left(N_{3}^{\mathrm{sph},-1}\right)^{2}, \\
& -4(1-w) \Delta_{\mathbb{C}^{6}}^{\mathrm{sph}}=-1+\mathcal{C}_{13}^{\mathrm{sph},-1}+\left(N_{2}^{\mathrm{sph},-1}\right)^{2}, \\
& -4 w \Delta_{\mathbb{C}^{6}}^{\mathrm{sph}}=-1+\mathcal{C}_{23}^{\mathrm{sph},-1}+\left(N_{1}^{\mathrm{sph},-1}\right)^{2} .
\end{aligned}
$$

They yield the factorizations of the balanced hypergeometric operator described in Section 2.1. Applying (7.12), we also obtain the factorizations of the standard hypergeometric operator described in [6].

\subsection{Conformal symmetries of $\Delta_{\mathbb{C}^{4}}$}

In this subsection we describe the reduction of the Laplacian on $\mathbb{C}^{6}$ to $\mathbb{C}^{4}$, which is accomplished by aplying the flat section. This will lead us to an alternative derivation of the hypergeometric equation. Besides, the material of this subsection will be needed when we will discuss the confluent equation. 
To a large extent, this subsection is a specification of Section 6.7 to $n=4$. Recall that the flat section is given by

$$
z_{-3}=-z_{-1} z_{1}-z_{-2} z_{2}, \quad z_{3}=1
$$

It is parametrized with $y \in \mathbb{C}^{4}$. More precisely, we introduce the coordinates

$$
y_{-1}=z_{-1}, \quad y_{1}=z_{1}, \quad y_{-2}=z_{-2}, \quad y_{2}=z_{2} .
$$

Thus this section can be identified with $\mathbb{C}^{4}$ with the scalar product given by the square

$$
\langle y \mid y\rangle=2 y_{-1} y_{1}+2 y_{-2} y_{2} .
$$

Lie algebra so $\left(\mathbb{C}^{6}\right)$. Cartan algebra:

$$
\begin{aligned}
& N_{i}^{\mathrm{fl}}=y_{-i} \partial_{y_{-i}}-y_{i} \partial_{y_{i}}, \quad i=1,2, \\
& N_{3}^{\mathrm{fl}, \eta}=y_{-1} \partial_{y_{-1}}+y_{1} \partial_{y_{1}}+y_{-2} \partial_{y_{-2}}+y_{2} \partial_{y_{2}}-\eta .
\end{aligned}
$$

Root operators:

$$
\begin{aligned}
& B_{1,2}^{\mathrm{fl}}=y_{-1} \partial_{y_{2}}-y_{-2} \partial_{y_{1}}, \quad B_{-1,-2}^{\mathrm{fl}}=y_{1} \partial_{y_{-2}}-y_{2} \partial_{y_{-1}}, \\
& B_{1,-2}^{\mathrm{fl}}=y_{-1} \partial_{y_{-2}}-y_{2} \partial_{y_{1}}, \quad B_{-1,2}^{\mathrm{fl}}=y_{1} \partial_{y_{2}}-y_{-2} \partial_{y_{-1}}, \\
& B_{3,1}^{\mathrm{fl}, \eta}=y_{-1}\left(\partial_{y_{-1}}-\eta\right)-y_{-2} y_{2} \partial_{y_{1}}+y_{-1} y_{-2} \partial_{y_{-2}}+y_{-1} y_{2} \partial_{y_{2}}, \\
& B_{-3,-1}^{\mathrm{fl}}=\partial_{y_{-1}}, \quad B_{3,-1}^{\mathrm{fl}, \eta}=y_{1}\left(\partial_{y_{1}}-\eta\right)-y_{-2} y_{2} \partial_{y_{-1}}+y_{1} y_{-2} \partial_{y_{-2}}+y_{1} y_{2} \partial_{y_{2}}, \\
& B_{-3,1}^{\mathrm{fl}}=\partial_{y_{1}}, \quad B_{3,2}^{\mathrm{fl}, \eta}=y_{-2}\left(\partial_{y_{-2}}-\eta\right)-y_{-1} y_{1} \partial_{y_{2}}+y_{-2} y_{-1} \partial_{y_{-1}}+y_{-2} y_{1} \partial_{y_{1}}, \\
& B_{-3,-2}^{\mathrm{fl}}=\partial_{y_{-2}}, \quad B_{3,-2}^{\mathrm{fl}, \eta}=y_{2}\left(\partial_{y_{2}}-\eta\right)-y_{-1} y_{1} \partial_{y_{-2}}+y_{2} y_{-1} \partial_{y_{-1}}+y_{2} y_{1} \partial_{y_{1}}, \\
& B_{-3,2}^{\mathrm{fl}}=\partial_{y_{2}} .
\end{aligned}
$$

Weyl symmetries. Transpositions:

$$
\begin{aligned}
\sigma_{(12)}^{\mathrm{fl}} f\left(y_{-1}, y_{1}, y_{-2}, y_{2}\right) & =f\left(y_{-2}, y_{2}, y_{-1}, y_{1}\right), \\
\sigma_{(13)}^{\mathrm{fl}, \eta} f\left(y_{-1}, y_{1}, y_{-2}, y_{2}\right) & =y_{1}^{\eta} f\left(\frac{-y_{-1} y_{1}-y_{-2} y_{2}}{y_{1}}, \frac{1}{y_{1}}, \frac{y_{-2}}{y_{1}}, \frac{y_{2}}{y_{1}}\right), \\
\sigma_{(23)}^{\mathrm{fl}, \eta} f\left(y_{-1}, y_{1}, y_{-2}, y_{2}\right) & =y_{2}^{\eta} f\left(\frac{y_{-1}}{y_{2}}, \frac{y_{1}}{y_{2}}, \frac{-y_{-1} y_{1}-y_{-2} y_{2}}{y_{2}}, \frac{1}{y_{2}}\right) .
\end{aligned}
$$

Cycles:

$$
\begin{aligned}
\sigma_{(123)}^{\mathrm{fl}, \eta} f\left(y_{-1}, y_{1}, y_{-2}, y_{2}\right) & =y_{2}^{\eta} f\left(\frac{-y_{-1} y_{1}-y_{-2} y_{2}}{y_{2}}, \frac{1}{y_{2}}, \frac{y_{-1}}{y_{2}}, \frac{y_{1}}{y_{2}}\right), \\
\sigma_{(132)}^{\mathrm{fl} l, \eta} f\left(y_{-1}, y_{1}, y_{-2}, y_{2}\right) & =y_{1}^{\eta} f\left(\frac{y_{-2}}{y_{1}}, \frac{y_{2}}{y_{1}}, \frac{-y_{-1} y_{1}-y_{-2} y_{2}}{y_{1}}, \frac{1}{y_{1}}\right) .
\end{aligned}
$$

Flips:

$$
\begin{aligned}
\tau_{1}^{\mathrm{fl}} f\left(y_{-1}, y_{1}, y_{-2}, y_{2}\right) & =f\left(y_{1}, y_{-1}, y_{-2}, y_{2}\right), \\
\tau_{2}^{\mathrm{fl}} f\left(y_{-1}, y_{1}, y_{-2}, y_{2}\right) & =f\left(y_{-1}, y_{1}, y_{2}, y_{-2}\right), \\
\tau_{3}^{\mathrm{fl}, \eta} f\left(y_{-1}, y_{1}, y_{-2}, y_{2}\right) & =\left(-2 y_{-1} y_{1}-2 y_{-2} y_{2}\right)^{\eta} f\left(\frac{y_{-1}, y_{1}, y_{-2}, y_{2}}{-y_{-1} y_{1}-y_{-2} y_{2}}\right) .
\end{aligned}
$$


Reduced Laplacian coincides with the 4-dimensional Laplacian:

$$
\Delta_{\mathbb{C}^{6}}^{\mathrm{fl}}=\Delta_{\mathbb{C}^{4}}=2 \partial_{y_{-1}} \partial_{y_{1}}+2 \partial_{y_{-2}} \partial_{y_{2}}
$$

\section{Generalized symmetries:}

$$
\begin{aligned}
N_{i}^{\mathrm{fl},-3} \Delta_{\mathbb{C}^{4}} & =\Delta_{\mathbb{C}^{4}} N_{i}^{\mathrm{fl},-1}, & & 1 \leq i \leq 3, \\
B_{i, j}^{\mathrm{fl},-3} \Delta_{\mathbb{C}^{4}} & =\Delta_{\mathbb{C}^{4}} B_{i, j}^{\mathrm{fl},-1}, & & 1 \leq|i|<|j| \leq 3, \\
\sigma^{\mathrm{fl},-3} \Delta_{\mathbb{C}^{4}} & =\Delta_{\mathbb{C}^{4}} \sigma^{\mathrm{fl},-1}, & & \sigma \in S_{3}, \\
\tau_{j}^{\mathrm{fl} l-3} \Delta_{\mathbb{C}^{4}} & =\Delta_{\mathbb{C}^{4}} \tau_{j}^{\mathrm{fl},-1}, & & 1 \leq j \leq 3 .
\end{aligned}
$$

\section{7 $\quad$ Deriving balanced hypergeometric operator from $\Delta_{\mathbb{C}^{4}}$}

Introduce the following coordinates in $\mathbb{C}^{4}$ :

$$
w=\frac{y_{-1} y_{1}}{y_{-1} y_{1}+y_{-2} y_{2}}, \quad r=\sqrt{2\left(y_{-1} y_{1}+y_{-2} y_{2}\right)}, \quad u_{1}=\sqrt{\frac{y_{-1}}{y_{1}}}, \quad u_{2}=\sqrt{\frac{y_{-2}}{y_{2}}} .
$$

We check that

$$
\begin{aligned}
& N_{1}^{\mathrm{fl}}=u_{1} \partial_{u_{1}}, \quad N_{2}^{\mathrm{fl}}=u_{2} \partial_{u_{2}}, \quad N_{3}^{\mathrm{fl}, \eta}=r \partial_{r}-\eta \\
& \Delta_{\mathbb{C}^{4}}=\frac{1}{r^{2}}\left(\left(r \partial_{r}+1\right)^{2}-1+4 \partial_{w} w(1-w) \partial_{w}-\frac{\left(u_{1} \partial_{u_{1}}\right)^{2}}{w}-\frac{\left(u_{2} \partial_{u_{2}}\right)^{2}}{(1-w)}\right) .
\end{aligned}
$$

Thus the ansatz

$$
f\left(w, u_{1}, u_{2}, r\right)=u_{1}^{\alpha} u_{2}^{\beta} r^{\mu-1} F(w)
$$

leads to the balanced hypergeometric operator.

\subsection{Deriving standard hypergeometric operator from $\Delta_{\mathbb{C}^{4}}$}

Alternatively, we can slightly change the coordinates (7.18), replacing $u_{1}, u_{2}$ with

$$
\tilde{u}_{1}:=\frac{y_{-1}}{\sqrt{y_{-1} y_{1}+y_{-2} y_{2}}}=u_{1} \sqrt{w}, \quad \tilde{u}_{2}:=\frac{y_{-2}}{\sqrt{y_{-1} y_{1}+y_{-2} y_{2}}}=u_{2} \sqrt{1-w} .
$$

We check that

$$
\begin{aligned}
N_{1}^{\mathrm{fl}}= & \tilde{u}_{1} \partial_{\tilde{u}_{1}}, \quad N_{2}^{\mathrm{fl}}=\tilde{u}_{2} \partial_{\tilde{u}_{2}}, \quad N_{3}^{\mathrm{fl}, \eta}=r \partial_{r}-\eta \\
\Delta_{\mathbb{C}^{4}}= & \frac{1}{r^{2}}\left(\left(r \partial_{r}+1\right)^{2}-1+4 w(1-w) \partial_{w}^{2}\right. \\
& \left.+4\left(\left(1+u_{1} \partial_{u_{1}}\right)(1-w)-\left(1+u_{2} \partial_{u_{2}}\right) w\right) \partial_{w}-\left(u_{1} \partial_{u_{1}}+u_{2} \partial_{u_{2}}+1\right)^{2}\right) .
\end{aligned}
$$

Thus the ansatz

$$
f\left(w, \tilde{u}_{1}, \tilde{u}_{2}, r\right)=\tilde{u}_{1}^{\alpha} \tilde{u}_{2}^{\beta} r^{\mu-1} F(w)
$$

leads to the standard hypergeometric equation. 


\section{$8 \operatorname{so}\left(\mathbb{C}^{5}\right)$ and the Gegenbauer equation}

In this section we derive the Gegenbauer operator and its so $\left(\mathbb{C}^{5}\right)$ symmetries. The whole section is very similar to Section 7 , where we derived the hypergeometric operator with its $\operatorname{so}\left(\mathbb{C}^{6}\right)$ symmetries. The main difference is lower dimension.

We will consider the following levels:

(1) extended space $\mathbb{C}^{5}$ and the Laplacian $\Delta_{\mathbb{C}^{5}}$,

(2) reduction to the so-called spherical section and the corresponding Laplace-Beltrami operator,

(3) depending on the choice of coordinates, separation of variables leads to the balanced or standard Gegenbauer operator.

There exists an alternative derivation:

$(2)^{\prime}$ reduction to $\mathbb{C}^{3}$ and $\Delta_{\mathbb{C}^{3}}$ with help of the flat section,

$(3)^{\prime}$ with appropriate coordinates, separation of variables leads to the balanced or standard Gegenbauer operator.

Some of the aspects of the Gegenbauer equation are actually more complicated than the corresponding aspects of the hypergeometric equation. This is seen, in particular, when we consider factorizations of the Gegenbauer operator, which come in two separate varieties, unlike for the hypergeometric operator, which has a single variety of factorizations. This corresponds to the fact that so(6) is simply-laced, whereas so(5) is not, i.e., its root operators are not of equal length.

\subsection{Extended space $\mathbb{C}^{5}$}

We consider $\mathbb{C}^{5}$ with the coordinates

$$
z_{0}, z_{-2}, z_{2}, z_{-3}, z_{3}
$$

and the scalar product given by

$$
\langle z \mid z\rangle=z_{0}^{2}+2 z_{-2} z_{2}+2 z_{-3} z_{3} .
$$

Note that we omit the indices $-1,1$; this makes it easier to compare $\mathbb{C}^{5}$ with $\mathbb{C}^{6}$.

Lie algebra $\mathrm{so}\left(\mathbb{C}^{5}\right)$. Cartan algebra:

$$
N_{i}=z_{-i} \partial_{z_{-i}}-z_{i} \partial_{z_{i}}, \quad i=2,3 .
$$

Root operators:

$$
B_{i, j}=z_{-i} \partial_{j}-z_{-j} \partial_{i}, \quad|i|=2, \quad|j|=3, \quad B_{0, j}=z_{0} \partial_{j}-z_{-j} \partial_{0}, \quad|j|=2,3 .
$$

Weyl symmetries. Transposition:

$$
\sigma_{(23)} K\left(z_{0}, z_{-2}, z_{2}, z_{-3}, z_{3}\right)=K\left(z_{0}, z_{-3}, z_{3}, z_{-2}, z_{2}\right) .
$$

Reflection and flips:

$$
\begin{aligned}
& \tau_{0} K\left(z_{0}, z_{-2}, z_{2}, z_{-3}, z_{3}\right)=K\left(-z_{0}, z_{2}, z_{-2}, z_{-3}, z_{3}\right) \\
& \tau_{2} K\left(z_{0}, z_{-2}, z_{2}, z_{-3}, z_{3}\right)=K\left(z_{0}, z_{2}, z_{-2}, z_{-3}, z_{3}\right) \\
& \tau_{3} K\left(z_{0}, z_{-2}, z_{2}, z_{-3}, z_{3}\right)=K\left(z_{0}, z_{-2}, z_{2}, z_{3}, z_{-3}\right)
\end{aligned}
$$




\section{Laplacian:}

$$
\Delta_{\mathbb{C}^{5}}=\partial_{z_{0}}^{2}+2 \partial_{z_{-2}} \partial_{z_{2}}+2 \partial_{z_{-3}} \partial_{z_{3}} .
$$

\section{Generalized symmetries:}

$$
\begin{array}{ll}
N_{i} \Delta_{\mathbb{C}^{5}}=\Delta_{\mathbb{C}^{5}} N_{i}, & i=2,3, \\
B_{i, j} \Delta_{\mathbb{C}^{5}}=\Delta_{\mathbb{C}^{5}} B_{i, j}, & |i|=2, \quad|j|=3, \\
B_{0, j} \Delta_{\mathbb{C}^{5}}=\Delta_{\mathbb{C}^{5}} B_{0, j}, & |j|=2,3, \\
\sigma_{(23)} \Delta_{\mathbb{C}^{5}}=\Delta_{\mathbb{C}^{5}} \sigma_{(23)}, & \\
\tau_{j} \Delta_{\mathbb{C}^{5}}=\Delta_{\mathbb{C}^{5}} \tau_{j}, & j=0,2,3 .
\end{array}
$$

\subsection{Spherical section}

We consider the section of the quadric

$$
\mathcal{V}^{4}:=\left\{z \in \mathbb{C}^{5}: z_{0}^{2}+2 z_{-2} z_{2}+2 z_{-3} z_{3}=0\right\}
$$

given by equations

$$
1=z_{0}^{2}+2 z_{-2} z_{2}=-2 z_{3} z_{-3} .
$$

We will call it the spherical section, because it is $\mathcal{S}^{2}(1) \times \mathcal{S}^{1}(-1)$. The superscript used for this section will be "sph" for spherical.

Introduce the following coordinates in $\mathbb{C}^{5}$ :

$$
\begin{array}{ll}
r=\sqrt{z_{0}^{2}+2 z_{-2} z_{2}}, & p=\sqrt{2 z_{3} z_{-3}}, \\
w=\sqrt{\frac{z_{0}^{2}}{2 z_{-2} z_{2}+z_{0}^{2}}}, \quad u_{2}=\sqrt{\frac{z_{-2}}{z_{2}}}, \quad u_{3}=\sqrt{\frac{z_{-3}}{z_{3}}} .
\end{array}
$$

Similarly as in the previous section, the null quadric in these coordinates is given by $r^{2}+p^{2}=0$. The generator of dilations is

$$
D_{\mathbb{C}^{5}}=r \partial_{r}+p \partial_{p} .
$$

The spherical section is given by the condition $r^{2}=1$.

Below we describe various objects in the spherical section.

Lie algebra $\operatorname{so}\left(\mathbb{C}^{5}\right)$. Cartan operators:

$$
N_{2}^{\mathrm{sph}}=u_{2} \partial_{u_{2}}, \quad N_{3}^{\mathrm{sph}}=u_{3} \partial_{u_{3}} .
$$

Root operators:

$$
\begin{aligned}
& B_{3,2}^{\mathrm{sph}, \eta}=\mathrm{i} u_{2} u_{3} \frac{\sqrt{1-w^{2}}}{2}\left(\eta-w \partial_{w}-\frac{N_{2}^{\mathrm{sph}}}{1-w^{2}}-N_{3}^{\mathrm{sph}}\right), \\
& B_{-3,-2}^{\mathrm{sph},}=\mathrm{i} \frac{1}{u_{2} u_{3}} \frac{\sqrt{1-w^{2}}}{2}\left(\eta-w \partial_{w}+\frac{N_{2}^{\mathrm{sph}}}{1-w^{2}}+N_{3}^{\mathrm{sph}}\right), \\
& B_{3,-2}^{\mathrm{sph}, \eta}=\mathrm{i} \frac{u_{3}}{u_{2}} \frac{\sqrt{1-w^{2}}}{2}\left(\eta-w \partial_{w}+\frac{N_{2}^{\mathrm{sph}}}{1-w^{2}}-N_{3}^{\mathrm{sph}}\right),
\end{aligned}
$$




$$
\begin{aligned}
& B_{-3,2}^{\mathrm{sph}, \eta}=\mathrm{i} \frac{u_{2}}{u_{3}} \frac{\sqrt{1-w^{2}}}{2}\left(\eta-w \partial_{w}-\frac{N_{2}^{\mathrm{sph}}}{1-w^{2}}+N_{3}^{\mathrm{sph}}\right), \\
& B_{3,0}^{\mathrm{sph}, \eta}=\mathrm{i} u_{3} \frac{w}{\sqrt{2}}\left(\eta+\frac{1-w^{2}}{w} \partial_{w}-N_{3}^{\mathrm{sph}}\right), \\
& B_{-3,0}^{\mathrm{sph}, \eta}=\mathrm{i} \frac{1}{u_{3}} \frac{w}{\sqrt{2}}\left(\eta+\frac{1-w^{2}}{w} \partial_{w}+N_{3}^{\mathrm{sph}}\right), \\
& B_{2,0}=u_{2} \sqrt{\frac{1-w^{2}}{2}}\left(\partial_{w}+\frac{w}{1-w^{2}} N_{2}^{\mathrm{sph}}\right), \\
& B_{-2,0}=\frac{1}{u_{2}} \sqrt{\frac{1-w^{2}}{2}}\left(\partial_{w}-\frac{w}{1-w^{2}} N_{2}^{\mathrm{sph}}\right) .
\end{aligned}
$$

Weyl symmetries. Transpositions:

$$
\sigma_{23}^{\mathrm{sph}, \eta} f\left(w, u_{2}, u_{3}\right)=\left(\mathrm{i} \sqrt{1-w^{2}}\right)^{\eta} f\left(\frac{w}{\sqrt{w^{2}-1}}, u_{3}, u_{2}\right) .
$$

Reflection and flips:

$$
\begin{aligned}
\tau_{0}^{\mathrm{sph}} f\left(w, u_{2}, u_{3}\right) & =f\left(-w, u_{2}, u_{3}\right), \\
\tau_{2}^{\mathrm{sph}} f\left(w, u_{2}, u_{3}\right) & =f\left(w, \frac{1}{u_{2}}, u_{3}\right), \quad \tau_{3}^{\mathrm{sph}} f\left(w, u_{2}, u_{3}\right)=f\left(w, u_{2}, \frac{1}{u_{3}}\right) .
\end{aligned}
$$

The Laplacian in coordinates is

$$
\Delta_{\mathbb{C}^{5}}=\frac{1}{r^{2}}\left(\left(r \partial_{r}\right)^{2}+\left(r \partial_{r}\right)+\frac{r^{2}}{p^{2}}\left(p \partial_{p}\right)^{2}+\partial_{w}\left(1-w^{2}\right) \partial_{w}-\frac{\left(u_{2} \partial_{u_{1}}\right)^{2}}{1-w^{2}}-\frac{r^{2}}{p^{2}}\left(u_{3} \partial_{u_{3}}\right)^{2}\right) .
$$

Using $r^{2}+p^{2}=1$ and

$$
\begin{aligned}
\left(r \partial_{r}\right)^{2}+r \partial_{r}+\frac{r^{2}}{p^{2}}\left(p \partial_{p}\right)^{2}= & \left(r \partial_{r}+\frac{r^{2}}{p^{2}}\left(p \partial_{p}\right)+\frac{1}{2}\right)\left(r \partial_{r}+p \partial_{p}+\frac{1}{2}\right)-\frac{1}{4} \\
& +\left(\frac{r^{2}}{p^{2}}+1\right)\left(r \partial_{r}+\frac{1}{2}\right)\left(p \partial_{p}\right) .
\end{aligned}
$$

we obtain

$$
\Delta_{\mathbb{C}^{5}}^{\diamond}=\frac{1}{r^{2}}\left(\partial_{w}\left(1-w^{2}\right) \partial_{w}-\frac{\left(u_{2} \partial_{u_{1}}\right)^{2}}{1-w^{2}}+\left(u_{3} \partial_{u_{3}}\right)^{2}-\frac{1}{4}\right) .
$$

To convert $\Delta_{\mathbb{C}^{5}}^{\diamond}$ into the reduced Laplacian $\Delta_{\mathbb{C}^{5}}^{\mathrm{sph}}$ we simply remove $\frac{1}{r^{2}}$, obtaining the LaplaceBeltrami operator on $\mathcal{S}^{2}(1) \times \mathcal{S}(1)$ :

$$
\Delta_{\mathbb{C}^{5}}^{\mathrm{sph}}=\partial_{w}\left(1-w^{2}\right) \partial_{w}-\frac{\left(N_{2}^{\mathrm{sph}}\right)^{2}}{1-w^{2}}+\left(N_{3}^{\mathrm{sph}}\right)^{2}-\frac{1}{4}
$$

We have

$$
\begin{array}{ll}
N_{i}^{\mathrm{sph}} \Delta_{\mathbb{C}^{3}}=\Delta_{\mathbb{C}^{3}} N_{i}^{\mathrm{sph}}, & i=2,3, \\
B_{i, j}^{\mathrm{sph},-\frac{5}{2}} \Delta_{\mathbb{C}^{3}}=\Delta_{\mathbb{C}^{3}} B_{i, j}^{\mathrm{sph},-\frac{1}{2}}, & |i|=2, \quad|j|=3, \\
B_{0, j}^{\mathrm{sph},-\frac{5}{2}} \Delta_{\mathbb{C}^{3}}=\Delta_{\mathbb{C}^{3}} B_{0, j}^{\mathrm{sph},-\frac{1}{2}}, & |j|=2,3, \\
\sigma_{(23)}^{\mathrm{sph},-\frac{5}{2}} \Delta_{\mathbb{C}^{3}}=\Delta_{\mathbb{C}^{3}} \sigma_{(23)}^{\mathrm{sph},-\frac{1}{2}}, & \\
\tau_{j}^{\mathrm{sph}} \Delta_{\mathbb{C}^{3}}=\Delta_{\mathbb{C}^{3}} \tau_{j}^{\mathrm{sph}}, & j=0,2,3 .
\end{array}
$$




\subsection{Balanced Gegenbauer operator}

Using the spherical section we make an ansatz

$$
f\left(w, u_{2}, u_{3}\right)=u_{2}^{\alpha} u_{3}^{\lambda} F(w) .
$$

Clearly,

$$
N_{2}^{\mathrm{sph}} f=\alpha f, \quad N_{3}^{\mathrm{sph}} f=\lambda f .
$$

Therefore, on functions of the form $(8.9), \Delta_{\mathbb{C}^{5}}^{\text {sph }}(8.3)$ coincides with the balanced Gegenbauer operator (2.4). The generalized symmetries for the roots (8.5) and (8.6), for the permutation (8.7), and for the flips (8.8) coincide with the transmutation relations, the discrete symmetries, and the sign changes of $\alpha, \lambda$ of the balanced Gegenbauer operator, respectively; see Section 2.2.

\subsection{Standard Gegenbauer operator}

Alternatively, we can replace the coordinate $u_{2}$ with

$$
\tilde{u}_{2}:=\frac{z_{-2}}{\sqrt{z_{0}^{2}+2 z_{-2} z_{2}}}=u_{2} \sqrt{\frac{1-w^{2}}{2}} .
$$

As compared with the previous coordinates, we need to replace $\partial_{w}$ with

$$
\partial_{w}-\frac{w}{\sqrt{1-w^{2}}} N_{2}^{\mathrm{sph}}
$$

In these coordinates

$$
\begin{aligned}
& N_{2}^{\mathrm{sph}}=\tilde{u}_{2} \partial_{\tilde{u}_{2}}, \quad N_{3}^{\mathrm{sph}}=u_{3} \partial_{u_{3}}, \\
& \Delta_{\mathbb{C}^{5}}^{\mathrm{sph}}=\left(1-w^{2}\right) \partial_{w}^{2}-2\left(1+N_{2}^{\mathrm{sph}}\right) w \partial_{w}+\left(N_{3}^{\mathrm{sph}}\right)^{2}-\left(N_{2}^{\mathrm{sph}}+\frac{1}{2}\right)^{2} .
\end{aligned}
$$

We make the ansatz

$$
f\left(w, u_{2}, u_{3}\right)=\tilde{u}_{2}^{\alpha} u_{3}^{\lambda} F(w) .
$$

Clearly,

$$
N_{2}^{\mathrm{sph}} f=\alpha f, \quad N_{3}^{\mathrm{sph}} f=\lambda f .
$$

Therefore, on functions of the form $(8.10), \Delta_{\mathbb{C}^{5}}^{\text {sph }}$ coincides with the standard Gegenbauer operator.

\subsection{Factorizations}

In the Lie algebra $\operatorname{so}\left(\mathbb{C}^{5}\right)$ with the coordinates $z_{0}, z_{-2}, z_{2}, z_{-3}, z_{3}$ we have 3 distinguished Lie subalgebras: one isomorphic to so $\left(\mathbb{C}^{4}\right)$ and two isomorphic to so $\left(\mathbb{C}^{3}\right)$. In an obvious notation,

$$
\mathrm{SO}_{23}\left(\mathbb{C}^{4}\right), \quad \mathrm{SO}_{02}\left(\mathbb{C}^{3}\right), \quad \mathrm{SO}_{03}\left(\mathbb{C}^{3}\right) .
$$

By (6.25) and (6.24), the corresponding Casimir operators are

$$
\begin{aligned}
\mathcal{C}_{23} & =4 B_{2,3} B_{-2,-3}-\left(N_{2}+N_{3}\right)^{2}-2 N_{2}-2 N_{3}=4 B_{-2,-3} B_{2,3}-\left(N_{2}+N_{3}\right)^{2}+2 N_{2}+2 N_{3} \\
& =4 B_{2,-3} B_{-2,3}-\left(N_{2}-N_{2}\right)^{2}-2 N_{2}+2 N_{3}=4 B_{-2,3} B_{2,-3}-\left(N_{2}-N_{3}\right)^{2}+2 N_{2}-2 N_{3}, \\
\mathcal{C}_{02} & =2 B_{0,2} B_{0,-2}-N_{2}^{2}-N_{2}=2 B_{0,2} B_{0,-2}-N_{2}^{2}+N_{2}, \\
\mathcal{C}_{03} & =2 B_{0,3} B_{0,-3}-N_{3}^{2}-N_{3}=2 B_{0,3} B_{0,-3}-N_{3}^{2}+N_{3} .
\end{aligned}
$$


After the reduction described in (6.18) and (6.19), we obtain the identities

$$
\begin{aligned}
& \left(2 z_{-2} z_{2}+2 z_{-3} z_{3}\right) \Delta_{\mathbb{C}^{5}}^{\diamond}=-\frac{3}{4}+\mathcal{C}_{23}^{\diamond,-\frac{1}{2}}, \\
& \left(z_{0}^{2}+2 z_{-2} z_{2}\right) \Delta_{\mathbb{C}^{5}}^{\diamond}=-\frac{1}{4}+\mathcal{C}_{02}^{\diamond,-\frac{1}{2}}+\left(N_{3}^{\diamond,-\frac{1}{2}}\right)^{2}, \\
& \left(z_{0}^{2}+2 z_{-3} z_{3}\right) \Delta_{\mathbb{C}^{5}}^{\diamond}=-\frac{1}{4}+\mathcal{C}_{03}^{\diamond,-\frac{1}{2}}+\left(N_{2}^{\diamond,-\frac{1}{2}}\right)^{2} .
\end{aligned}
$$

If we use the spherical section, (8.11a), (8.11b), (8.11c) become

$$
\begin{aligned}
& -w^{2} \Delta_{\mathbb{C}^{5}}^{\mathrm{sph}}=-\frac{3}{4}+\mathcal{C}_{23}^{\mathrm{sph},-\frac{1}{2}}, \\
& \Delta_{\mathbb{C}^{5}}^{\mathrm{sph}}=-\frac{1}{4}+\mathcal{C}_{02}^{\mathrm{sph},-\frac{1}{2}}+\left(N_{3}^{\mathrm{sph}}\right)^{2}, \\
& \left(w^{2}-1\right) \Delta_{\mathbb{C}^{5}}^{\mathrm{sph}}=-\frac{1}{4}+\mathcal{C}_{03}^{\mathrm{sph},-\frac{1}{2}}+\left(N_{2}^{\mathrm{sph}}\right)^{2} .
\end{aligned}
$$

They yield the factorizations of the balanced Gegenbauer operator described in Section 2.2 and of the standard Gegenbauer operator described in [6].

\subsection{Conformal symmetries of $\Delta_{\mathbb{C}^{3}}$}

In this subsection we describe the reduction of the Laplacian on $\mathbb{C}^{5}$ to $\mathbb{C}^{3}$. To this end we apply the flat section. This will lead us to an alternative derivation of the Gegenbauer equation. Besides, the material of this subsection will be needed when we will discuss the Hermite equation.

To a large extent, this subsection is a specification of Section 6.7 to $n=3$. Recall that the flat section is given by

$$
z_{-3}=-\frac{1}{2} z_{0}^{2}-z_{-2} z_{2}, \quad z_{3}=1 .
$$

We introduce the coordinates

$$
y_{0}=z_{0}, \quad y_{-2}=z_{-2}, \quad y_{2}=z_{2} .
$$

Thus we obtain $\mathbb{C}^{3}$ with the scalar product given by

$$
\langle y \mid y\rangle=y_{0}^{2}+2 y_{-2} y_{2} .
$$

Lie algebra $\operatorname{so}\left(\mathbb{C}^{5}\right)$. Cartan operators:

$$
\begin{aligned}
& N_{2}^{\mathrm{fl}}=y_{-2} \partial_{y_{-2}}-y_{2} \partial_{y_{2}}, \\
& N_{3}^{\mathrm{fl}, \eta}=y_{0} \partial_{y_{0}}+y_{-2} \partial_{y_{-2}}+y_{2} \partial_{y_{2}}-\eta .
\end{aligned}
$$

Root operators:

$$
\begin{array}{lll}
B_{0,2}^{\mathrm{fl}}=y_{0} \partial_{y_{2}}-y_{-2} \partial_{y_{0}}, & B_{0,-2}^{\mathrm{fl}}=y_{0} \partial_{y_{-2}}-y_{2} \partial_{y_{0}}, & \\
B_{0,3}^{\mathrm{fl}}=y_{0} \partial_{y_{3}}-y_{-3} \partial_{y_{0}}, \quad B_{0,-3}^{\mathrm{fl}}=y_{0} \partial_{y_{-3}}-y_{3} \partial_{y_{0}}, & \\
B_{3,2}^{\mathrm{fl}, \eta}=y_{-2}\left(\partial_{y_{-2}}-\eta\right)-y_{-2} y_{2} \partial_{y_{2}}+y_{-2} y_{-2} \partial_{y_{-2}}+y_{-2} y_{2} \partial_{y_{2}}, & B_{-3,-2}^{\mathrm{fl}}=\partial_{y_{-2}}, \\
B_{3,-2}^{\mathrm{fl}, \eta}=y_{2}\left(\partial_{y_{2}}-\eta\right)-y_{-2} y_{2} \partial_{y_{-2}}+y_{2} y_{-2} \partial_{y_{-2}}+y_{2} y_{2} \partial_{y_{2}}, & B_{-3,2}^{\mathrm{fl}}=\partial_{y_{2}} .
\end{array}
$$


Weyl symmetries. Transpositions:

$$
\sigma_{(23)}^{\mathrm{fl}, \eta} f\left(y_{0}, y_{-2}, y_{2}\right)=y_{2}^{\eta} f\left(\frac{-\frac{1}{2} y_{0}^{2}-y_{-2} y_{2}}{y_{2}}, \frac{1}{y_{2}}\right) \text {. }
$$

Flips:

$$
\begin{aligned}
& \tau_{0}^{\mathrm{fl}} f\left(y_{0}, y_{-2}, y_{2}\right)=f\left(y_{0}, y_{2}, y_{-2}\right), \quad \tau_{2}^{\mathrm{fl}} f\left(y_{0}, y_{-2}, y_{2}\right)=f\left(y_{0}, y_{2}, y_{-2}\right), \\
& \tau_{3}^{\mathrm{fl}, \eta} f\left(y_{0}, y_{-2}, y_{2}\right)=\left(-y_{0}^{2}-2 y_{-2} y_{2}\right)^{\eta} f\left(\frac{y_{0}, y_{-2}, y_{2}}{-\frac{1}{2} y_{0}^{2}-y_{-2} y_{2}}\right) .
\end{aligned}
$$

Reduced Laplacian coincides with the 3-dimensional Laplacian:

$$
\Delta_{\mathbb{C}^{3}}^{\mathrm{fl}}=\Delta_{\mathbb{C}^{3}}=\partial_{y_{0}}^{2}+2 \partial_{y_{-2}} \partial_{y_{2}} .
$$

\section{Generalized symmetries:}

$$
\begin{aligned}
& N_{i}^{\mathrm{fl},-\frac{5}{2}} \Delta_{\mathbb{C}^{3}}=\Delta_{\mathbb{C}^{3}} N_{i}^{\mathrm{fl},-\frac{1}{2}}, \quad i=2,3, \\
& B_{i, j}^{\mathrm{fl},-\frac{5}{2}} \Delta_{\mathbb{C}^{3}}=\Delta_{\mathbb{C}^{3}} B_{i, j}^{\mathrm{fl},-\frac{1}{2}}, \quad|i|=2, \quad|j|=3, \\
& B_{0, j}^{\mathrm{fl},-\frac{5}{2}} \Delta_{\mathbb{C}^{3}}=\Delta_{\mathbb{C}^{3}} B_{0, j}^{\mathrm{fl},-\frac{1}{2}}, \quad|j|=2,3, \\
& \sigma_{(23)}^{\mathrm{fl},-\frac{5}{2}} \Delta_{\mathbb{C}^{3}}=\Delta_{\mathbb{C}^{3}} \sigma_{(23)}^{\mathrm{fl},-\frac{1}{2}}, \\
& \tau_{j}^{\mathrm{fl},-\frac{5}{2}} \Delta_{\mathbb{C}^{3}}=\Delta_{\mathbb{C}^{3}} \tau_{j}^{\mathrm{fl},-\frac{1}{2}}, \quad j=0,2,3 .
\end{aligned}
$$

\subsection{Deriving balanced Gegenbauer operator from $\Delta_{\mathbb{C}^{3}}$}

Introduce the following coordinates in $\mathbb{C}^{3}$ :

$$
u:=\sqrt{\frac{y_{-2}}{y_{2}}}, \quad r:=\sqrt{y_{0}^{2}+2 y_{-2} y_{2}}, \quad w:=\frac{y_{0}}{\sqrt{y_{0}^{2}+2 y_{-2} y_{2}}} .
$$

Clearly,

$$
\begin{aligned}
& N_{2}^{\mathrm{fl}}=u \partial_{u}, \quad N_{3}^{\mathrm{fl}, \eta}=r \partial_{r}-\eta \\
& \Delta_{\mathbb{C}^{3}}=\frac{1}{r^{2}}\left(\partial_{w}\left(1-w^{2}\right) \partial_{w}-\frac{\left(u \partial_{u}\right)^{2}}{1-w^{2}}+\left(r \partial_{r}+\frac{1}{2}\right)^{2}-\frac{1}{4}\right) .
\end{aligned}
$$

Thus the ansatz

$$
f(w, u, r)=u^{\alpha} r^{\lambda-\frac{1}{2}} F(w)
$$

leads to the balanced Gegenbauer operator (2.4).

\subsection{Deriving standard Gegenbauer operator from $\Delta_{\mathbb{C}^{3}}$}

Instead of the coordinate $u$ choose

$$
\tilde{u}:=\frac{y_{-2}}{\sqrt{y_{0}^{2}+2 y_{-2} y_{2}}}=u \sqrt{\frac{1-w^{2}}{2}} .
$$


Clearly,

$$
\begin{aligned}
& N_{2}^{\mathrm{fl}}=\tilde{u} \partial_{\tilde{u}}, \quad N_{3}^{\mathrm{fl}, \eta}=r \partial_{r}-\eta \\
& \Delta_{\mathbb{C}^{3}}=\frac{1}{r^{2}}\left(\left(1-w^{2}\right) \partial_{w}^{2}-2\left(1+N_{2}^{\mathrm{fl}}\right) w \partial_{w}-\left(\tilde{u} \partial_{\tilde{u}}+\frac{1}{2}\right)^{2}+\left(r \partial_{r}+\frac{1}{2}\right)^{2}\right) .
\end{aligned}
$$

Thus the ansatz

$$
f(w, \tilde{u}, r)=\tilde{u}^{\alpha} r^{\lambda-\frac{1}{2}} \tilde{F}(w)
$$

leads to the standard Gegenbauer operator (2.3).

\section{Symmetries of the heat equation - the Schrödinger algebra}

The main subject of this section are generalized (infinitesimal) symmetries of the heat equation

$$
\left(\Delta_{\mathbb{C}^{n-2}}+\partial_{t}\right) f=0
$$

We will see in particular that the Lie group of generalized symmetries of $(9.1)$ is $\operatorname{sch}\left(\mathbb{C}^{n-2}\right)$, the so-called Schrödinger Lie algebra.

We will reduce the heat equation (9.1) to the Laplace equation on $\mathbb{C}^{n}(6.1)$, whose Lie algebra of generalized infinitesimal symmetries is, as we saw, $\operatorname{so}\left(\mathbb{C}^{n+2}\right) \cdot \operatorname{sch}\left(\mathbb{C}^{n-2}\right)$ can be viewed as a subalgebra of so $\left(\mathbb{C}^{n+2}\right)$.

Note that the choice of the dimension $n-2$ in (9.1) makes our presentation of the heat equation consistent with that of the Laplace equation of Section 6. It will be convenient to start again from the extended space $\mathbb{C}^{n+2}$, where all symmetries greatly simplify.

\subsection{The Schrödinger Lie algebra and group on $\mathbb{C}^{n+2}$}

We consider again the space $\mathbb{C}^{n+2}$ with the scalar product given by

$$
\langle z \mid z\rangle=\sum_{i \in I_{n+2}} z_{-i} z_{i}, \quad z \in \mathbb{C}^{n+2},
$$

and the Laplacian

$$
\Delta_{\mathbb{C}^{n+2}}=\sum_{i \in I_{n+2}} \partial_{z_{-i}} \partial_{z_{i}}
$$

Recall that the Lie algebra so $\left(\mathbb{C}^{n+2}\right)$ and the group $\mathrm{O}\left(\mathbb{C}^{n+2}\right)$ have natural representations on $\mathbb{C}^{n+2}(6.2)$ and (6.3) commuting with $\Delta_{\mathbb{C}^{n+2}}$, see (6.4), (6.5). A special role will be played by the operator

$$
B_{-m-1, m}=z_{m+1} \partial_{z_{m}}-z_{-m} \partial_{z_{-m-1}} \in \mathrm{so}\left(\mathbb{C}^{n+2}\right) .
$$

We define the Schrödinger Lie algebra

$$
\operatorname{sch}\left(\mathbb{C}^{n-2}\right):=\left\{B \in \operatorname{so}\left(\mathbb{C}^{n+2}\right):\left[B, B_{-m-1, m}\right]=0\right\} .
$$

We also have the full and special Schrödinger group

$$
\begin{aligned}
& \operatorname{Sch}\left(\mathbb{C}^{n-2}\right):=\left\{\alpha \in \mathrm{O}\left(\mathbb{C}^{n+2}\right): \alpha B_{-m-1, m}=B_{-m-1, m} \alpha\right\} \\
& \operatorname{SSch}\left(\mathbb{C}^{n-2}\right):=\operatorname{Sch}\left(\mathbb{C}^{n-2}\right) \cap \operatorname{SO}\left(\mathbb{C}^{n+2}\right) .
\end{aligned}
$$




\subsection{Structure of $\operatorname{sch}\left(\mathbb{C}^{n-2}\right)$}

Let us describe the structure of $\operatorname{sch}\left(\mathbb{C}^{n-2}\right)$.

We will use our usual notation for elements of so $\left(\mathbb{C}^{n+2}\right)$ and $\mathrm{O}\left(\mathbb{C}^{n+2}\right)$. In particular,

$$
N_{m}=z_{-m} \partial_{z_{-m}}-z_{m} \partial_{z_{m}}, \quad N_{m+1}=z_{-m-1} \partial_{z_{-m-1}}-z_{m+1} \partial_{z_{m+1}} .
$$

Define

$$
N_{m, m+1}:=N_{m}+N_{m+1} .
$$

Note that $N_{m, m+1}$ belongs to $\operatorname{sch}\left(\mathbb{C}^{n-2}\right)$ and commutes with so $\left(\mathbb{C}^{n-2}\right)$, which is naturally embedded in $\operatorname{sch}\left(\mathbb{C}^{n-2}\right)$. $\operatorname{sch}\left(\mathbb{C}^{n-2}\right)$ is spanned by the following operators:

(1) $B_{-m-1, m}$, which spans the center of $\operatorname{sch}\left(\mathbb{C}^{n-2}\right)$,

(2) $B_{m, j}, B_{-m-1, j}, j=1, \ldots, m-1$, which have the following nonzero commutator:

$$
\left[B_{m, j}, B_{-m-1,-j}\right]=B_{-m-1, m},
$$

(3) $B_{-m-1,-m}, B_{m+1, m}, N_{m, m+1}$, which have the usual commutation relations of $\operatorname{sl}\left(\mathbb{C}^{2}\right) \simeq$ so $\left(\mathbb{C}^{3}\right)$ :

$$
\left[B_{m+1, m}, B_{-m-1,-m}\right]=N_{m . m+1}, \quad\left[B_{ \pm(m+1), \pm m}, N_{m, m+1}\right]= \pm B_{ \pm(m+1), \pm m},
$$

(4) $B_{i, j},|i|<|j| \leq m-1, N_{i}, i=1, \ldots, m-1$, with the usual commutation relations of $\operatorname{so}\left(\mathbb{C}^{n-2}\right)$.

The span of (2) can be identified with $\mathbb{C}^{n-2} \oplus \mathbb{C}^{n-2} \simeq \mathbb{C}^{2} \otimes \mathbb{C}^{n-2}$, which has a natural structure of a symplectic space. The span of (1) and (2) is the central extension of the abelian algebra $\mathbb{C}^{2} \otimes \mathbb{C}^{n-2}$ by (9.2). Such a Lie algebra is usually called the Heisenberg Lie algebra over $\mathbb{C}^{2} \otimes \mathbb{C}^{n-2}$ and can be denoted by

$$
\text { heis }\left(\mathbb{C}^{2} \otimes \mathbb{C}^{n-2}\right)=\mathbb{C} \rtimes\left(\mathbb{C}^{2} \otimes \mathbb{C}^{n-2}\right) \text {. }
$$

$\operatorname{sl}\left(\mathbb{C}^{2}\right)$ acts in the obvious way on $\mathbb{C}^{2}$ and $\operatorname{so}\left(\mathbb{C}^{n-2}\right)$ acts on $\mathbb{C}^{n-2}$. Thus $\operatorname{sl}\left(\mathbb{C}^{2}\right) \oplus \operatorname{so}\left(\mathbb{C}^{n-2}\right)$ acts on $\mathbb{C}^{2} \otimes \mathbb{C}^{n-2}$. Thus

$$
\operatorname{sch}\left(\mathbb{C}^{n-2}\right) \simeq \mathbb{C} \rtimes\left(\mathbb{C}^{2} \otimes \mathbb{C}^{n-2}\right) \rtimes\left(\operatorname{sl}\left(\mathbb{C}^{2}\right) \oplus \operatorname{so}\left(\mathbb{C}^{n-2}\right)\right) .
$$

Note, in particular, that neither $\operatorname{sch}\left(\mathbb{C}^{n-2}\right) \operatorname{nor} \operatorname{SSch}\left(\mathbb{C}^{n-2}\right)$ are semisimple.

The subalgebra spanned by the usual Cartan algebra of so $\left(\mathbb{C}^{n-2}\right), N_{m, m+1}$ and $B_{-m-1, m}$ is a maximal commutative subalgebra of $\operatorname{sch}\left(\mathbb{C}^{n-2}\right)$. It will be called the "Cartan algebra" of $\operatorname{sch}\left(\mathbb{C}^{n-2}\right)$.

Let us introduce $\kappa \in \mathrm{SO}\left(\mathbb{C}^{n-2} \oplus \mathbb{C}^{2} \oplus \mathbb{C}^{2}\right)$ :

$$
\kappa\left(\ldots, z_{-m}, z_{m}, z_{-m-1}, z_{m+1}\right):=\left(\ldots, z_{m+1}, z_{-m-1},-z_{m},-z_{-m}\right) .
$$

Note that $\kappa^{4}=\mathrm{id}$ and $\kappa \in \mathrm{SSch}\left(\mathbb{C}^{n-2}\right)$. On the level of functions

$$
\kappa K\left(\ldots, z_{-m}, z_{m}, z_{-m-1}, z_{m+1}\right):=K\left(\ldots,-z_{m+1},-z_{-m-1}, z_{m}, z_{-m}\right) .
$$

The subgroup of $\operatorname{Sch}\left(\mathbb{C}^{n-2}\right)$ generated by $W\left(\mathbb{C}^{n-2}\right) \subset \mathrm{O}\left(\mathbb{C}^{n-2}\right)$ and $\kappa$ will be called the group of Weyl symmetries of $\operatorname{sch}\left(\mathbb{C}^{n-2}\right)$. 


\subsection{The Schrödinger Lie algebra and group on $\mathbb{C}^{n}$}

Recall that in Section 6.7 we used the decomposition $\mathbb{C}^{n+2}=\mathbb{C}^{n} \oplus \mathbb{C}^{2}$. Elements of $\mathbb{C}^{n}$ were generically denoted by $y$. The space $\mathbb{C}^{n}$ will be also useful in this section. Further on, it will be decomposed as $\mathbb{C}^{n}=\mathbb{C}^{n-2} \oplus \mathbb{C}^{2}$. Thus the square of an element of $\mathbb{C}^{n}$ is equal to

$$
\langle y \mid y\rangle_{\mathbb{C}^{n}}=\langle y \mid y\rangle_{\mathbb{C}^{n-2}}+2 y_{-m} y_{m}, \quad y \in \mathbb{C}^{n},
$$

and the Laplacian

$$
\Delta_{\mathbb{C}^{n}}=\Delta_{\mathbb{C}^{n-2}}+2 \partial_{y_{-m}} \partial_{y_{m}}
$$

Recall that we have the representations

$$
\begin{aligned}
& \operatorname{so}\left(\mathbb{C}^{n+2}\right) \ni B \mapsto B^{\mathrm{fl}, \eta} \in \mathcal{A} \rtimes \operatorname{hol}\left(\mathbb{C}^{n}\right), \\
& \mathrm{O}\left(\mathbb{C}^{n+2}\right) \ni \alpha \mapsto \alpha^{\mathrm{fl}, \eta} \underset{\text { loc }}{\in} \mathcal{A}^{\times} \rtimes \operatorname{Hol}\left(\mathbb{C}^{n}\right),
\end{aligned}
$$

and the generalized symmetry

$$
\begin{array}{ll}
B^{\mathrm{fl}, \frac{-2-n}{2}} \Delta_{\mathbb{C}^{n}}=\Delta_{\mathbb{C}^{n}} B^{\mathrm{fl}, \frac{2-n}{2}}, & B \in \mathrm{So}\left(\mathbb{C}^{n+2}\right), \\
\alpha^{\mathrm{fl}, \frac{-2-n}{2}} \Delta_{\mathbb{C}^{n}}=\Delta_{\mathbb{C}^{n}} \alpha^{\mathrm{fl}, \frac{2-n}{2}}, & \alpha \in \mathrm{O}\left(\mathbb{C}^{n+2}\right) .
\end{array}
$$

\subsection{The Schrödinger Lie algebra and group on $\mathbb{C}^{n-2} \oplus \mathbb{C}$}

We consider now the space $\mathbb{C}^{n-2} \oplus \mathbb{C}$ with the generic variables $(y, t)=\left(\ldots, y_{m-1}, t\right)$. Note that $t$ should be understood as a new name for $y_{-m}$, and we keep the old names for the first $n-2$ coordinates.

We define the map $\theta: \mathcal{A}\left(\mathbb{C}^{n-2} \oplus \mathbb{C}\right) \rightarrow \mathcal{A}\left(\mathbb{C}^{n}\right)$ by setting for $h$

$$
(\theta h)\left(\ldots, y_{m-1}, y_{-m}, y_{m}\right):=h\left(\ldots, y_{m-1}, y_{-m}\right) \mathrm{e}^{y_{m}} .
$$

We also define $\zeta: \mathcal{A}\left(\mathbb{C}^{n}\right) \rightarrow \mathcal{A}\left(\mathbb{C}^{n-2} \oplus \mathbb{C}\right)$, which to $f$ associates

$$
(\zeta f)\left(\ldots, y_{m-1}, t\right):=f\left(\ldots, y_{m-1}, t, 0\right) \text {. }
$$

Clearly, $\zeta$ is a left inverse of $\theta$ :

$$
\zeta \circ \theta=\mathrm{id}
$$

Therefore, $\theta \circ \zeta=\mathrm{id}$ is true on the range of $\theta$.

The heat operator in $n-2$ spatial dimensions can be obtained from the Laplacian in $n$ dimension:

$$
\mathcal{L}_{\mathbb{C}^{n-2}}:=\Delta_{\mathbb{C}^{n-2}}+2 \partial_{t}=\zeta \Delta_{\mathbb{C}^{n}} \theta
$$

For $B \in \operatorname{sch}\left(\mathbb{C}^{n-2}\right) \subset \operatorname{so}\left(\mathbb{C}^{n+2}\right)$ we define

$$
B^{\mathrm{sch}, \eta}:=\zeta B^{\mathrm{fl}, \eta} \theta,
$$

and for $\alpha \in \operatorname{Sch}\left(\mathbb{C}^{n-2}\right) \subset \mathrm{O}\left(\mathbb{C}^{n+2}\right)$,

$$
\alpha^{\mathrm{sch}, \eta}:=\zeta \alpha^{\mathrm{fl}, \eta} \theta \text {. }
$$

Lemma 9.1. $\operatorname{sch}\left(\mathbb{C}^{n-2}\right), \operatorname{Sch}\left(\mathbb{C}^{n-2}\right)$ and $\Delta_{\mathbb{C}^{n}}$ preserve the range of $\theta$. 
Proof. Note that

$$
B_{-m-1, m}^{\mathrm{fl}, \eta}=\partial_{y_{m}} .
$$

Let $B \in \operatorname{sch}\left(\mathbb{C}^{n-2}\right)$. Then $\left[B^{\mathrm{fl}, \eta}, \partial_{y_{m}}\right]=0$. Therefore,

$$
B^{\mathrm{fl}, \eta}=C+D \partial_{y_{m}},
$$

where $C \in \operatorname{hol}\left(\mathbb{C}^{n-2} \oplus \mathbb{C}\right)$ and $D \in \mathcal{A}\left(\mathbb{C}^{n-2} \oplus \mathbb{C}\right)$ (they do not involve the variable $\left.y_{m}\right)$. Therefore, $B$ preserves the range of $\theta$.

Likewise, if $\alpha \in \operatorname{Sch}\left(\mathbb{C}^{n-2}\right)$, then we have

$$
\alpha^{\mathrm{fl}, \eta} f\left(\ldots, y_{-m}, y_{m}\right)=\beta f\left(\ldots, y_{-m}, y_{m}+d\left(\ldots, y_{-m}\right)\right),
$$

where $\beta \in \mathcal{A}^{\times} \rtimes \operatorname{Hol}\left(\mathbb{C}^{n} \oplus \mathbb{C}\right), d \in \mathcal{A}\left(\mathbb{C}^{n-2} \oplus \mathbb{C}\right)$ (they do not involve the variable $\left.y_{m}\right)$. Therefore, $\alpha^{\mathrm{fl}, \eta}$ preserves the range of $\theta$.

For $\Delta_{\mathbb{C}^{n}}$ the statement is contained in the formula (9.5).

\section{Theorem 9.2.}

(1) For any $\eta$,

$$
\begin{aligned}
& \operatorname{sch}\left(\mathbb{C}^{n-2}\right) \ni B \mapsto B^{\text {sch }, \eta} \in \mathcal{A} \rtimes \operatorname{hol}\left(\mathbb{C}^{n-2} \oplus \mathbb{C}\right), \\
& \operatorname{Sch}\left(\mathbb{C}^{n-2}\right) \ni \alpha \mapsto \alpha^{\text {sch, }, \eta} \underset{\text { loc }}{\in} \mathcal{A}^{\times} \rtimes \operatorname{Hol}\left(\mathbb{C}^{n-2} \oplus \mathbb{C}\right)
\end{aligned}
$$

is a representation/local representation.

(2) We have a generalized symmetry

$$
\begin{aligned}
B^{\mathrm{sch}, \frac{-2-n}{2}} \mathcal{L}_{\mathbb{C}^{n-2}}=\mathcal{L}_{\mathbb{C}^{n-2}} B^{\mathrm{sch}, \frac{2-n}{2}}, & B \in \operatorname{sch}\left(\mathbb{C}^{n-2}\right), \\
\alpha^{\mathrm{sch}, \frac{-2-n}{2}} \mathcal{L}_{\mathbb{C}^{n-2}}=\mathcal{L}_{\mathbb{C}^{n-2}} \alpha^{\mathrm{sch}, \frac{2-n}{2}}, &
\end{aligned}
$$

Proof. Let us first prove (1). Obviously, for $B_{1}, B_{2} \in \mathrm{so}\left(\mathbb{C}^{n+2}\right)$

$$
\zeta\left[B_{1}^{\mathrm{fl}, \eta} B_{2}^{\mathrm{fl}, \eta}\right] \theta=\zeta\left[B_{1}, B_{2}\right]^{\mathrm{fl}, \eta} \theta .
$$

If $B_{1}, B_{2} \in \operatorname{sch}\left(\mathbb{C}^{n-2}\right)$, we can insert $\theta \circ \zeta$ in the middle of the commutator. Hence

$$
\left[B_{1}^{\mathrm{sch}, \eta}, B_{2}^{\mathrm{sch}, \eta}\right]=\left[B_{1}, B_{2}\right]^{\mathrm{sch}, \eta} .
$$

Now, for any $\alpha_{1}, \alpha_{2} \in \mathrm{O}\left(\mathbb{C}^{n+2}\right)$

$$
\zeta \alpha_{1}^{\mathrm{fl}, \eta} \alpha_{2}^{\mathrm{fl}, \eta} \theta=\zeta\left(\alpha_{1} \alpha_{2}\right)^{\mathrm{fl}, \eta} \theta .
$$

For $\alpha_{1}, \alpha_{2} \in \operatorname{Sch}\left(\mathbb{C}^{n-2}\right)$ we can insert $\theta \circ \zeta$ in the middle of the composition, obtaining

$$
\alpha_{1}^{\mathrm{sch}, \eta} \alpha_{2}^{\mathrm{sch}, \eta}=\left(\alpha_{1} \alpha_{2}\right)^{\mathrm{sch}, \eta} .
$$

To prove (2) we multiply (9.3) and (9.4) by $\zeta$ from the left and $\theta$ from the right:

$$
\begin{array}{rlrl}
\zeta B^{\mathrm{fl}, \frac{-2-n}{2}} \Delta_{\mathbb{C}^{n}} \theta & =\zeta \Delta_{\mathbb{C}^{n}} B^{\mathrm{fl}, \frac{2-n}{2}} \theta, & & B \in \mathrm{so}\left(\mathbb{C}^{n+2}\right), \\
\zeta \alpha^{\mathrm{fl}, \frac{-2-n}{2}} \Delta_{\mathbb{C}^{n}} \theta=\zeta \Delta_{\mathbb{C}^{n}} \alpha^{\mathrm{fl} l, \frac{2-n}{2}} \theta, & & \alpha \in \mathrm{O}\left(\mathbb{C}^{n+2}\right) .
\end{array}
$$

For $B \in \operatorname{sch}\left(\mathbb{C}^{n-2}\right)$ and $\alpha \in \operatorname{Sch}\left(\mathbb{C}^{n-2}\right)$ we can insert $\theta \circ \zeta$, which yields $(2)$. 


\subsection{Hermite operator}

Consider again the space $\mathbb{C}^{n-2} \oplus \mathbb{C}$. This time its generic coordinates will be denoted $(w, s)$. We assume that the space $\mathbb{C}^{n-2}$ is equipped with a scalar product. The following operator can be called the $(n-2)$-dimensional Hermite operator:

$$
\mathcal{H}_{\mathbb{C}^{n-2}}:=\Delta_{\mathbb{C}^{n-2}}-D_{\mathbb{C}^{n-2}}+s \partial_{s}
$$

The heat operator is closely related to the Hermite operator. Indeed, let us change the coordinates from $(y, t) \in \mathbb{C}^{n-2} \oplus \mathbb{C}^{\times}$to $(w, s) \in \mathbb{C}^{n-2} \oplus \mathbb{C}^{\times}$by

$$
w=t^{-\frac{1}{2}} y, \quad s=t^{\frac{1}{2}},
$$

with the inverse transformation

$$
y=w s, \quad t=s^{2} .
$$

Under this transformation the heat operator $\mathcal{L}_{\mathbb{C}^{n-2}}$ becomes $\frac{1}{s^{2}} \mathcal{H}_{\mathbb{C}^{n-2}}$.

In Section 11 we will use this change of coordinates to obtain the (1-dimensional) Hermite operator. The construction is, however, interesting in higher dimensions as well, therefore we mention it here.

Strictly speaking, the above coordinate change does not work globally: in particular, we need to assume $s \neq 0, t \neq 0$, besides $s$ doubly covers $t$. We usually are not absolutely precise about specifying the domains of coordinate changes - if needed, the reader can easily fill in such details.

\subsection{Schrödinger symmetries in coordinates}

In this subsection we sum up information about Schrödinger symmetries on 4 levels described in the previous subsections. Note that the last two levels differ only by a change of coordinates. Therefore, the operators on these two levels are denoted by the same symbols, with the same superscript ${ }^{\text {sch }}$.

We start with generic names of the variables and the corresponding squares:

$$
\begin{array}{ll}
z \in \mathbb{C}^{n+2}, & \langle z \mid z\rangle_{\mathbb{C}^{n+2}}=\sum_{j \in I_{n+2}} z_{-j} z_{j}, \\
y \in \mathbb{C}^{n}, & \langle y \mid y\rangle_{\mathbb{C}^{n}}=\sum_{j \in I_{n}} y_{-j} y_{j}, \\
(y, t) \in \mathbb{C}^{n-2} \oplus \mathbb{C}, & \langle y \mid y\rangle_{\mathbb{C}^{n-2}}=\sum_{j \in I_{n-2}} y_{-j} y_{j}, \\
(w, s) \in \mathbb{C}^{n-2} \oplus \mathbb{C}, & \langle w \mid w\rangle_{\mathbb{C}^{n-2}}=\sum_{j \in I_{n-2}} w_{-j} w_{j} .
\end{array}
$$

Cartan algebra of $\operatorname{sch}\left(\mathbb{C}^{n-2}\right)$. Central element:

$$
\begin{aligned}
& B_{-m-1, m}=z_{m+1} \partial_{z_{m}}-z_{-m} \partial_{z_{-m-1}}, \\
& B_{-m-1, m}^{\mathrm{fl}}=\partial_{y_{m}}, \quad B_{-m-1, m}^{\mathrm{sch}}=1, \quad B_{-m-1, m}^{\mathrm{sch}}=1 .
\end{aligned}
$$

Cartan algebra of $\mathrm{so}\left(\mathbb{C}^{n-2}\right), j=1, \ldots, m-1$ :

$$
\begin{array}{ll}
N_{j}=z_{-j} \partial_{z_{-j}}-z_{j} \partial_{z_{j}}, & N_{j}^{\mathrm{fl}}=y_{-j} \partial_{y_{-j}}-y_{j} \partial_{y_{j}}, \\
N_{j}^{\mathrm{sch}}=y_{-j} \partial_{y_{-j}}-y_{j} \partial_{y_{j}}, & N_{j}^{\mathrm{sch}}=w_{-j} \partial_{w_{-j}}-w_{j} \partial_{w_{j}} .
\end{array}
$$


Generator of scaling:

$$
\begin{aligned}
& N_{m, m+1}=z_{-m} \partial_{z_{-m}}-z_{m} \partial_{z_{m}}+z_{-m-1} \partial_{z_{-m-1}}-z_{m+1} \partial_{z_{m+1}}, \\
& N_{m, m+1}^{\mathrm{fl}, \eta}=\sum_{j \in I_{n-2}} y_{j} \partial_{y_{j}}+2 y_{-m} \partial_{y_{-m}}-\eta, \\
& N_{m, m+1}^{\mathrm{sch}, \eta}=\sum_{j \in I_{n-2}} y_{j} \partial_{y_{j}}+2 t \partial_{t}-\eta, \quad N_{m, m+1}^{\mathrm{sch}, \eta}=s \partial_{s}-\eta .
\end{aligned}
$$

Root operators of $\operatorname{sch}\left(\mathbb{C}^{n-2}\right)$. Roots of $\operatorname{so}\left(\mathbb{C}^{n-2}\right),|i|<|j|, i, j \in I_{n-2}$ :

$$
\begin{array}{ll}
B_{i, j}=z_{-i} \partial_{z_{j}}-z_{-j} \partial_{z_{i}}, & B_{i, j}^{\mathrm{fl}}=y_{-i} \partial_{y_{j}}-y_{-j} \partial_{y_{i}}, \\
B_{i, j}^{\mathrm{sch}}=y_{-i} \partial_{y_{j}}-y_{-j} \partial_{y_{i}}, & B_{i, j}^{\mathrm{sch}}=w_{-i} \partial_{w_{j}}-w_{-j} \partial_{w_{i}} .
\end{array}
$$

Space translations, $j \in I_{n-2}$ :

$$
\begin{aligned}
& B_{-m-1, j}=z_{m+1} \partial_{z_{j}}-z_{-j} \partial_{z_{-m-1}}, \\
& B_{-m-1, j}^{\mathrm{fl}}=\partial_{y_{j}}, \quad B_{-m-1, j}^{\mathrm{sch}}=\partial_{y_{j}}, \quad B_{-m-1, j}^{\mathrm{sch}}=\frac{1}{s} \partial_{w_{j}} .
\end{aligned}
$$

Time translation:

$$
\begin{aligned}
& B_{-m-1,-m}=z_{m+1} \partial_{z_{-m}}-z_{m} \partial_{z_{-m-1}}, \quad B_{-m-1,-m}^{\mathrm{fl}}=\partial_{y_{-m}}, \quad B_{-m-1,-m}^{\mathrm{sch}}=\partial_{t}, \\
& B_{-m-1,-m}^{\mathrm{sch}}=\frac{1}{2 s^{2}}\left(-\sum_{j \in I_{n-2}} w_{j} \partial_{w_{j}}+s \partial_{s}\right) .
\end{aligned}
$$

Additional roots, $j \in I_{n-2}$ :

$$
\begin{aligned}
& B_{m, j}=z_{-m} \partial_{z_{j}}-z_{-j} \partial_{z_{m}}, \quad B_{m, j}^{\mathrm{fl}}=y_{-m} \partial_{y_{j}}-y_{-j} \partial_{y_{m}}, \quad B_{m, j}^{\mathrm{sch}}=t \partial_{y_{j}}-y_{-j}, \\
& B_{m, j}^{\mathrm{sch}}=s\left(\partial_{w_{j}}-w_{-j}\right), \quad B_{m+1, m}=z_{-m-1} \partial_{z_{m}}-z_{-m} \partial_{z_{m+1}}, \\
& B_{m+1, m}^{\mathrm{fl}, \eta}=y_{-m}\left(\sum_{j \in I_{n-2}} y_{j} \partial_{y_{j}}+y_{-m} \partial_{y_{-m}}-\eta\right)-\frac{1}{2} \sum_{j \in I_{n-2}} y_{-j} y_{j} \partial_{y_{m}}, \\
& B_{m+1, m}^{\mathrm{sch}, \eta}=t\left(\sum_{j \in I_{n-2}} y_{j} \partial_{y_{j}}+y_{-m} \partial_{y_{-m}}-\eta\right)-\frac{1}{2} \sum_{j \in I_{n-2}} y_{-j} y_{j}, \\
& B_{m+1, m}^{\mathrm{sch}, \eta}=\frac{s^{2}}{2}\left(s \partial_{s}-2 \eta+\sum_{j \in I_{n-2}} w_{j} \partial_{w_{j}}-\sum_{j \in I_{n-2}} w_{j} w_{-j}\right) .
\end{aligned}
$$

Weyl symmetries. We will write $K$ for a function on $\mathbb{C}^{n+2}, f$ for a function on $\mathbb{C}^{n}, h$ for a function on $\mathbb{C}^{n-2} \oplus \mathbb{C}$ in both coordinates $\left(\ldots, y_{m-1}, t\right)$ and $\left(\ldots, w_{m-1}, s\right)$.

Reflection:

$$
\begin{aligned}
& \tau_{0} K\left(z_{0}, \ldots, z_{-m}, z_{m}, z_{-m-1}, z_{m+1}\right)=K\left(-z_{0}, \ldots, z_{-m}, z_{m}, z_{-m-1}, z_{m+1}\right), \\
& \tau_{0}^{\mathrm{fl}} f\left(y_{0}, \ldots, y_{-m}, y_{m}\right)=f\left(-y_{0}, \ldots, y_{-m}, y_{m}\right), \\
& \tau_{0}^{\mathrm{sch}} h\left(y_{0}, \ldots, t\right)=h\left(-y_{0}, \ldots, t\right), \quad \tau_{0}^{\mathrm{sch}} h\left(w_{0}, \ldots, s\right)=h\left(-w_{0}, \ldots, s\right) .
\end{aligned}
$$

Flips, $j=1, \ldots, m-1$ :

$$
\begin{aligned}
& \tau_{j} K\left(\ldots, z_{-j}, z_{j}, \ldots, z_{-m}, z_{m}, z_{-m-1}, z_{m+1}\right)=K\left(\ldots, z_{j}, z_{-j}, \ldots, z_{-m}, z_{m}, z_{-m-1}, z_{m+1}\right), \\
& \tau_{j}^{\mathrm{fl}} f\left(\ldots, y_{-j}, y_{j}, \ldots, y_{-m}, y_{m}\right)=f\left(\ldots, y_{j}, y_{-j}, \ldots, y_{-m}, y_{m}\right), \\
& \tau_{j}^{\mathrm{sch}} h\left(\ldots, y_{-j}, y_{j}, \ldots, t\right)=h\left(\ldots, y_{j}, y_{-j}, \ldots, t\right), \\
& \tau_{j}^{\mathrm{sch}} h\left(\ldots, w_{-j}, w_{j}, \ldots, s\right)=h\left(\ldots, w_{j}, w_{-j}, \ldots, s\right) .
\end{aligned}
$$


Permutations, $\sigma \in S_{m-1}$ :

$$
\begin{aligned}
& \sigma K\left(\ldots, z_{-m+1}, z_{m-1}, z_{-m}, z_{m}, z_{-m-1}, z_{m+1}\right) \\
& \quad=K\left(\ldots, z_{-\sigma_{m-1}}, z_{\sigma_{m-1}}, z_{-m}, z_{m}, z_{-m-1}, z_{m+1}\right), \\
& \sigma^{\mathrm{fl}} f\left(\ldots, y_{-m+1}, y_{m-1}, y_{-m}, y_{m}\right)=f\left(\ldots, y_{-\sigma_{m-1}}, y_{\sigma_{m-1}}, y_{-m}, y_{m}\right), \\
& \sigma^{\mathrm{sch}} h\left(\ldots, y_{-m+1}, y_{m-1}, t\right)=h\left(\ldots, y_{-\sigma_{m-1}}, y_{\sigma_{m-1}}, t\right), \\
& \sigma^{\mathrm{sch}} h\left(\ldots, w_{-m+1}, w_{m-1}, s\right)=h\left(\ldots, w_{-\sigma_{m-1}}, w_{\sigma_{m-1}}, s\right) .
\end{aligned}
$$

Special transformation $\kappa$ :

$$
\begin{aligned}
& \kappa K\left(\ldots, z_{m-1}, z_{-m}, z_{m}, z_{-m-1}, z_{m+1}\right)=K\left(\ldots, z_{m-1},-z_{m+1},-z_{-m-1}, z_{m}, z_{-m}\right), \\
& \kappa^{\mathrm{fl}, \eta} f\left(\ldots, y_{m-1}, y_{-m}, y_{m}\right)=y_{-m}^{\eta} f\left(\ldots, \frac{y_{m-1}}{y_{-m}},-\frac{1}{y_{-m}}, \frac{1}{2 y_{-m}} \sum_{j \in I_{n}} y_{-j} y_{j}\right), \\
& \kappa^{\mathrm{sch}, \eta} h\left(\ldots, y_{m-1}, t\right)=t^{\eta} \exp \left(\frac{1}{2 t} \sum_{j \in I_{n-2}} y_{-j} y_{j}\right) h\left(\ldots, \frac{y_{m-1}}{t},-\frac{1}{t}\right), \\
& \kappa^{\mathrm{sch}, \eta} h\left(\ldots, w_{m-1}, s\right)=s^{2 \eta} \exp \left(\frac{1}{2} \sum_{j \in I_{n-2}} w_{-j} w_{j}\right) h\left(\ldots,-\mathrm{i} w_{m-1}, \frac{\mathrm{i}}{s}\right) .
\end{aligned}
$$

Square of $\kappa$ :

$$
\begin{aligned}
& \kappa^{2} K\left(\ldots, z_{m-1}, z_{-m}, z_{m}, z_{-m-1}, z_{m+1}\right)=K\left(\ldots, z_{m-1},-z_{-m},-z_{m},-z_{-m-1},-z_{m+1}\right), \\
& \left(\kappa^{\mathrm{fl}, \eta}\right)^{2} f\left(\ldots, y_{m-1}, y_{-m}, y_{m}\right)=f\left(\ldots,-y_{m-1}, y_{-m}, y_{m}\right), \\
& \left(\kappa^{\mathrm{sch}, \eta}\right)^{2} h\left(\ldots, y_{m-1}, t\right)=(-1)^{\eta} h\left(\ldots,-y_{m-1}, t\right), \\
& \left(\kappa^{\mathrm{sch}, \eta}\right)^{2} h\left(\ldots, w_{m-1}, s\right)=(-1)^{\eta} h\left(\ldots,-w_{m-1}, s\right) .
\end{aligned}
$$

\section{Laplacian / Laplacian / heat operator / Hermite operator:}

$$
\begin{aligned}
& \Delta_{\mathbb{C}^{n+2}}=\sum_{j \in I_{n+2}} \partial_{z_{-j}} \partial_{z_{j}}, \quad \Delta_{\mathbb{C}^{n}}=\sum_{j \in I_{n}} \partial_{y_{-j}} \partial_{y_{j}}, \quad \mathcal{L}_{\mathbb{C}^{n-2}}=\sum_{j \in I_{n-2}} \partial_{y_{-j}} \partial_{y_{j}}+2 \partial_{t}, \\
& \frac{1}{s^{2}} \mathcal{H}_{\mathbb{C}^{n-2}}=\frac{1}{s^{2}}\left(\sum_{j \in I_{n-2}} \partial_{w_{-j}} \partial_{w_{j}}-\sum_{j \in I_{n-2}} w_{j} \partial_{w_{j}}+s \partial_{s}\right) .
\end{aligned}
$$

Computations. Let us sketch how we computed the Schrödinger Lie algebra and group in coordinates. We set

$$
\Phi^{\mathrm{sch}, \eta}:=\Phi^{\mathrm{fl}, \eta} \circ \theta, \quad \Psi^{\mathrm{sch}, \eta}:=\zeta \circ \Psi^{\mathrm{fl}, \eta} .
$$

Then $\Phi^{\text {sch }, \eta}$, maps $h \in \mathcal{A}\left(\mathbb{C}^{n-2} \oplus \mathbb{C}\right)$ onto

$$
\begin{aligned}
& \left(\Phi^{\mathrm{sch}, \eta} h\right)\left(\ldots, z_{-m+1}, z_{m-1}, z_{-m}, z_{m}, z_{-m-1}, z_{m+1}\right) \\
& \quad:=z_{m+1}^{\eta} h\left(\ldots, \frac{z_{-m+1}}{z_{m+1}}, \frac{z_{m-1}}{z_{m+1}}, \frac{z_{-m}}{z_{m+1}}\right) \exp \left(\frac{z_{m}}{z_{m+1}}\right) .
\end{aligned}
$$

For $K \in \mathcal{A}\left(\mathbb{C}^{n-2} \oplus \mathbb{C}^{2} \oplus \mathbb{C}^{2}\right)$

$$
\left(\Psi^{\mathrm{sch}, \eta} K\right)\left(\ldots, y_{-m+1}, y_{m-1}, t\right):=K\left(\ldots, y_{-m+1}, y_{m-1}, t, 0,-\frac{1}{2}\langle y \mid y\rangle, 1\right) .
$$


Note that

$$
\begin{aligned}
& \Psi^{\mathrm{sch}, \eta} \Phi^{\mathrm{sch}, \eta}=\mathrm{id}, \\
& \Psi^{\mathrm{sch}, \eta} \Delta_{\mathbb{C}^{n+2}} \Phi^{\mathrm{sch}, \eta}=\mathcal{L}_{\mathbb{C}^{n-2}}, \\
& \Psi^{\mathrm{sch}, \eta} B \Phi^{\mathrm{sch}, \eta}=B^{\mathrm{sch}, \eta}, \quad B \in \operatorname{sch}\left(\mathbb{C}^{n-2}\right), \\
& \Psi^{\mathrm{sch}, \eta} \alpha \Phi^{\mathrm{sch}, \eta}=\alpha^{\mathrm{sch}, \eta}, \quad \alpha \in \operatorname{Sch}\left(\mathbb{C}^{n-2}\right) .
\end{aligned}
$$

\section{$10 \operatorname{sch}\left(\mathbb{C}^{2}\right)$ and the confluent equation}

In this section we derive the confluent operator and its $\operatorname{sch}\left(\mathbb{C}^{2}\right)$ symmetries. We will consider the following levels:

(1) extended space $\mathbb{C}^{6}$ and the Laplacian,

(2) reduction to $\mathbb{C}^{4}$ and the Laplacian,

(3) reduction to $\mathbb{C}^{2} \oplus \mathbb{C}$ and the heat operator,

(4) special coordinates,

(5) sandwiching with a weight,

(6) depending on the choice of coordinates, separation of variables leads to the balanced or standard confluent operator.

A separate subsection will be devoted to factorizations of the confluent operator.

\section{$10.1 \mathbb{C}^{6}$}

We again consider $\mathbb{C}^{6}$ with the coordinates $(7.1)$ and the product given by (7.2). We describe various object related to the Lie algebra $\operatorname{sch}\left(\mathbb{C}^{2}\right)$. Remember that $\operatorname{sch}\left(\mathbb{C}^{2}\right)$ is a subalgebra of so $\left(\mathbb{C}^{6}\right)$ and we keep the notation from so $\left(\mathbb{C}^{6}\right)$.

Lie algebra $\operatorname{sch}\left(\mathbb{C}^{2}\right)$. Cartan algebra is spanned by

$$
\begin{aligned}
& N_{1}=z_{-1} \partial_{z_{-1}}-z_{1} \partial_{z_{1}}, \\
& N_{2,3}=z_{-2} \partial_{z_{-2}}-z_{2} \partial_{z_{2}}+z_{-3} \partial_{z_{-3}}-z_{3} \partial_{z_{3}}, \\
& B_{-3,2}=z_{3} \partial_{z_{2}}-z_{-2} \partial_{z_{-3}} .
\end{aligned}
$$

Root operators:

$$
\begin{array}{lll}
B_{2,-1}=z_{-2} \partial_{z_{-1}}-z_{1} \partial_{z_{2}}, & B_{2,1}=z_{-2} \partial_{z_{1}}-z_{-1} \partial_{z_{2}}, & B_{-3,-1}=z_{3} \partial_{z_{-1}}-z_{1} \partial_{z_{-3}}, \\
B_{-3,1}=z_{3} \partial_{z_{1}}-z_{-1} \partial_{z_{-3}}, & B_{-3,-2}=z_{3} \partial_{z_{-2}}-z_{2} \partial_{z_{-3}}, & B_{3,2}=z_{-3} \partial_{z_{2}}-z_{-2} \partial_{z_{3}} .
\end{array}
$$

Weyl symmetries. Special symmetry of order 4:

$$
\kappa K\left(z_{-1}, z_{1}, z_{-2}, z_{2}, z_{-3}, z_{3}\right)=K\left(z_{-1}, z_{1},-z_{3},-z_{-3}, z_{2}, z_{-2}\right) .
$$

Flip:

$$
\tau_{1} K\left(z_{-1}, z_{1}, z_{-2}, z_{2}, z_{-3}, z_{3}\right)=K\left(z_{1}, z_{-1}, z_{-2}, z_{2}, z_{-3}, z_{3}\right) .
$$

We also have the Laplacian (7.3) satisfying (7.4a)-(7.4d). 


\section{$10.2 \mathbb{C}^{4}$}

We descend on the level of $\mathbb{C}^{4}$, with the coordinates (7.16) and the scalar product given by (7.17).

Lie algebra $\operatorname{sch}\left(\mathbb{C}^{2}\right)$. Cartan algebra:

$$
N_{2,3}^{\mathrm{fl}, \eta}=y_{-1} \partial_{y_{-}}+y_{1} \partial_{y_{1}}+2 y_{-2} \partial_{y_{-2}}-\eta, \quad N_{1}^{\mathrm{fl}}=y_{-1} \partial_{y_{-}-1}-y_{1} \partial_{y_{1}}, \quad B_{-3,2}^{\mathrm{fl}}=\partial_{y_{2}} .
$$

Root operators:

$$
\begin{aligned}
& B_{2,-1}^{\mathrm{fl}}=y_{-2} \partial_{y_{-}}-y_{1} \partial_{y_{2}}, \quad B_{2,1}^{\mathrm{fl}}=y_{-2} \partial_{y_{1}}-y_{1} \partial_{y_{2}}, \quad B_{-3,-1}^{\mathrm{fl}}=\partial_{y_{-1}}, \quad B_{-3,1}^{\mathrm{fl}}=\partial_{y_{1}}, \\
& B_{-3,-2}^{\mathrm{fl}}=\partial_{y_{-2}}, \quad B_{3,2}^{\mathrm{fl}, \eta}=-y_{-1} y_{1} \partial_{y_{2}}+y_{-2}\left(y_{-1} \partial_{y_{-1}}+y_{1} \partial_{y_{1}}+y_{-2} \partial_{y_{-2}}-\eta\right) .
\end{aligned}
$$

Weyl symmetries. Special symmetry of order 4 :

$$
\kappa^{\mathrm{fl}, \eta} f\left(y_{-1}, y_{1}, y_{-2}, y_{2}\right)=y_{-2}^{\eta} f\left(\frac{y_{1}}{y_{-2}}, \frac{y_{-1}}{y_{-2}},-\frac{1}{y_{-2}}, \frac{2 y_{-1} y_{1}+2 y_{-2} y_{2}}{2 y_{-2}}\right) \text {. }
$$

Flip:

$$
\tau_{1}^{\mathrm{fl}} f\left(y_{-1}, y_{1}, y_{-2}, y_{2}\right)=f\left(y_{1}, y_{-1}, y_{-2}, y_{2}\right) .
$$

\section{$10.3 \mathbb{C}^{2} \oplus \mathbb{C}$}

We apply the ansatz involving the exponential $\mathrm{e}^{y_{2}}$. We rename $y_{-2}$ to $t$. The operator $B_{-3,2}^{\text {sch }}$ becomes equal to 1 , therefore it can be ignored further on.

Lie algebra $\operatorname{sch}\left(\mathbb{C}^{2}\right)$. Cartan algebra:

$$
N_{2,3}^{\mathrm{sch}, \eta}=y_{-1} \partial_{y_{-1}}+y_{1} \partial_{y_{1}}+2 t \partial_{t}-\eta, \quad N_{1}^{\mathrm{sch}}=y_{-1} \partial_{y_{-1}}-y_{1} \partial_{y_{1}} .
$$

Root operators:

$$
\begin{aligned}
& B_{2,-1}^{\mathrm{sch}}=t \partial_{y_{-1}}-y_{1}, \quad B_{2,1}^{\mathrm{sch}}=t \partial_{y_{1}}-y_{-1}, \quad B_{-3,-1}^{\mathrm{sch}}=\partial_{y_{-1}}, \quad B_{-3,1}^{\mathrm{sch}}=\partial_{y_{1}}, \\
& B_{-3,-2}^{\mathrm{sch}}=\partial_{t}, \quad B_{3,2}^{\mathrm{sch}, \eta}=-y_{-1} y_{1}+t\left(y_{-1} \partial_{y_{-1}}+y_{1} \partial_{y_{1}}+t \partial_{t}-\eta\right) .
\end{aligned}
$$

Weyl symmetries. Special symmetry of order 4 :

$$
\kappa^{\mathrm{sch}, \eta} h\left(y_{-1}, y_{1}, t\right)=t^{\eta} \exp \left(\frac{y_{-1} y_{1}}{t}\right) h\left(\frac{y_{-1}}{t}, \frac{y_{1}}{t},-\frac{1}{t}\right) .
$$

Flip:

$$
\tau_{1}^{\mathrm{sch}} h\left(y_{-1}, y_{1}, t\right)=h\left(y_{1}, y_{-1}, t\right) .
$$

\section{Heat operator:}

$$
\mathcal{L}_{\mathbb{C}^{2}}=2 \partial_{y_{-1}} \partial_{y_{1}}+2 \partial_{t} .
$$

\section{Generalized symmetries:}

$$
\begin{aligned}
& N_{1}^{\text {sch }} \mathcal{L}_{\mathbb{C}^{2}}=\mathcal{L}_{\mathbb{C}^{2}} N_{1}^{\text {sch }}, \\
& N_{2,3}^{\text {sch, }-3} \mathcal{L}_{\mathbb{C}^{2}}=\mathcal{L}_{\mathbb{C}^{2}} N_{23}^{\text {sch },-1}, \\
& B_{i, j}^{\text {sch,-3 }} \mathcal{L}_{\mathbb{C}^{2}}=\mathcal{L}_{\mathbb{C}^{2}} B_{i, j}^{\text {sch, }-1}, \quad(i, j)=(2, \pm 1),(-3, \pm 1), \pm(3,2) ; \\
& \kappa^{\text {sch, }-3} \mathcal{L}_{\mathbb{C}^{2}}=\mathcal{L}_{\mathbb{C}^{2}} \kappa^{\text {sch, }-1}, \\
& \tau_{1}^{\text {sch }} \mathcal{L}_{\mathbb{C}^{2}}=\mathcal{L}_{\mathbb{C}^{2}} \tau_{1}^{\text {sch }} .
\end{aligned}
$$




\subsection{Coordinates $u, w, s$}

Let us define new complex variables as

$$
w=\frac{y_{-1} y_{1}}{t}, \quad u=\sqrt{\frac{y_{-1}}{y_{1}}}, \quad s=\sqrt{t} .
$$

Here are the reverse transformations:

$$
y_{-1}=u s \sqrt{w}, \quad y_{1}=\frac{1}{u} s \sqrt{w}, \quad t=s^{2} .
$$

Lie algebra $\operatorname{sch}\left(\mathbb{C}^{2}\right)$. Cartan algebra:

$$
N_{2,3}^{\mathrm{sch}, \eta}=s \partial_{s}-\eta, \quad N_{1}^{\mathrm{sch}}=u \partial_{u} .
$$

Root operators:

$$
\begin{array}{ll}
B_{-3,-1}^{\mathrm{sch}}=\frac{1}{u s} \frac{1}{\sqrt{w}}\left(w \partial_{w}+\frac{N_{1}^{\mathrm{sch}}}{2}\right), & B_{-3,1}^{\mathrm{sch}}=\frac{u}{s} \frac{1}{\sqrt{w}}\left(w \partial_{w}-\frac{N_{1}^{\mathrm{sch}}}{2}\right), \\
B_{2,-1}^{\mathrm{sch}}=\frac{s}{u} \frac{1}{\sqrt{w}}\left(w \partial_{w}+\frac{N_{1}^{\mathrm{sch}}}{2}-w\right), & B_{2,1}^{\mathrm{sch}}=s u \frac{1}{\sqrt{w}}\left(w \partial_{w}-\frac{N_{1}^{\mathrm{sch}}}{2}-w\right), \\
B_{-3,-2}^{\mathrm{sch} \eta}=\frac{1}{s^{2}}\left(-w \partial_{w}+\frac{N_{23}^{\mathrm{sch}, \eta}}{2}+\frac{\eta}{2}\right), & B_{3,2}^{\mathrm{sch}, \eta}=s^{2}\left(w \partial_{w}+\frac{N_{23}^{\mathrm{sch}, \eta}}{2}-\frac{\eta}{2}-w\right) .
\end{array}
$$

Weyl symmetries. Special symmetry of order 4:

$$
\kappa^{\mathrm{sch}, \eta} h(w, u, s)=s^{2 \eta} \mathrm{e}^{w} h\left(-w, u, \frac{1}{s}\right) .
$$

Flip:

$$
\tau_{1}^{\mathrm{sch}} h(w, u, s)=h\left(w, \frac{1}{u}, s\right) .
$$

\section{Heat operator:}

$$
\mathcal{L}_{\mathbb{C}^{2}}=\frac{2}{s^{2}}\left(\partial_{w} w \partial_{w}-w \partial_{w}-\frac{\left(u \partial_{u}\right)^{2}}{4 w}+\frac{1}{2} s \partial_{s}\right) .
$$

\subsection{Sandwiching with an exponential}

For any operator $C$ we define

$$
\hat{C}:=\mathrm{e}^{-\frac{w}{2}} C \mathrm{e}^{\frac{w}{2}} .
$$

The "hat" isomorphism will not change the Cartan operators:

$$
\hat{N}_{2,3}^{\text {sch, } \eta}=s \partial_{s}-\eta, \quad \hat{N}_{1}^{\text {sch }}=u \partial_{u} .
$$

Root operators:

$$
\begin{array}{ll}
\hat{B}_{-3,-1}^{\mathrm{sch}}=\frac{1}{u s} \frac{1}{\sqrt{w}}\left(w \partial_{w}+\frac{\hat{N}_{1}^{\mathrm{sch}}}{2}+\frac{w}{2}\right), & \hat{B}_{-3,1}^{\mathrm{sch}}=\frac{u}{s} \frac{1}{\sqrt{w}}\left(w \partial_{w}-\frac{\hat{N}_{1}^{\mathrm{sch}}}{2}+\frac{w}{2}\right), \\
\hat{B}_{2,-1}^{\mathrm{sch}}=\frac{s}{u} \frac{1}{\sqrt{w}}\left(w \partial_{w}+\frac{\hat{N}_{1}^{\mathrm{sch}}}{2}-\frac{w}{2}\right), & \hat{B}_{2,1}^{\mathrm{sch}}=s u \frac{1}{\sqrt{w}}\left(w \partial_{w}-\frac{\hat{N}_{1}^{\mathrm{sch}}}{2}-\frac{w}{2}\right), \\
\hat{B}_{-3,-2}^{\mathrm{sch}, \eta}=\frac{1}{s^{2}}\left(-w \partial_{w}+\frac{\hat{N}_{2,3}^{\mathrm{sch}, \eta}}{2}+\frac{\eta}{2}-\frac{w}{2}\right), & \hat{B}_{3,2}^{\mathrm{sch}, \eta}=s^{2}\left(w \partial_{w}+\frac{\hat{N}_{2,3}^{\mathrm{sch}, \eta}}{2}-\frac{\eta}{2}-\frac{w}{2}\right) .
\end{array}
$$


Weyl symmetries. Special symmetry of order 4:

$$
\hat{\kappa}^{\mathrm{sch}, \eta} h(w, u, s)=s^{2 \eta} h\left(-w, u, \frac{1}{s}\right) .
$$

Flip:

$$
\hat{\tau}_{1}^{\mathrm{sch}} h(w, u, s)=h\left(w, \frac{1}{u}, s\right) .
$$

Heat operator.

$$
\hat{\mathcal{L}}_{\mathbb{C}^{2}}=\mathrm{e}^{-\frac{w}{2}} \mathcal{L}_{\mathbb{C}^{2}} \mathrm{e}^{\frac{w}{2}}=\frac{2}{s^{2}}\left(\partial_{w} w \partial_{w}-\frac{w}{4}-\frac{\left(\hat{N}_{1}^{\mathrm{sch}}\right)^{2}}{4 w}+\frac{1}{2} \hat{N}_{2,3}^{\mathrm{sch},-1}\right) .
$$

\subsection{Balanced confluent operator}

We make an ansatz

$$
h(w, u, s)=u^{\alpha} s^{-\theta-1} F(w) .
$$

Clearly,

$$
\hat{N}_{1}^{\mathrm{sch}} h=\alpha h, \quad \hat{N}_{2,3}^{\mathrm{sch},-1} h=-\theta h .
$$

Therefore, on functions of this form, $\frac{s^{2}}{2} \mathcal{L}_{\mathbb{C}^{2}}$ coincides with the balanced confluent operator (2.6). The generalized symmetries for the roots (10.3), for the special Weyl symmetry (10.4) and for the flip (10.5) coincide with the transmutation relations, the discrete symmetry and the sign changes of $\alpha, \theta$ of the balanced confluent operator, respectively; see Section 2.3.

\subsection{Standard confluent operator}

Let us change slightly coordinates by replacing $u$ with

$$
\tilde{u}:=\frac{y_{-1}}{\sqrt{t}}=u \sqrt{w}
$$

The derivative $\partial_{w}$ is then replaced by

$$
\partial_{w}+\frac{1}{2 w} N_{1}
$$

Let us make an ansatz

$$
h(w, \tilde{u}, s)=\tilde{u}^{\alpha} s^{-\theta-1} \tilde{F}(w) .
$$

Clearly,

$$
N_{1}^{\mathrm{sch}} h=\alpha h, \quad N_{2,3}^{\mathrm{sch},-1} h=-\theta h .
$$

Then, on functions of the form $(10.7), \frac{s^{2}}{2} \mathcal{L}_{\mathbb{C}^{2}}$ coincides with the standard confluent operator $(2.5)$. 


\subsection{Factorizations}

Let us note the commutation relation

$$
\left[B_{-3,2}, B_{3,2}\right]=N_{2}+N_{3}=N_{2,3} .
$$

It shows that the triple $B_{-3,2}, B_{3,2}$ and $N_{2,3}$ defines a subalgebra isomorphic to so $\left(\mathbb{C}^{3}\right)$, which we will denote $\mathrm{so}_{23}\left(\mathbb{C}^{3}\right)$. The Casimir operator for $\mathrm{so}_{23}\left(\mathbb{C}^{3}\right)$ is

$$
\mathcal{C}_{23}=4 B_{3,2} B_{-3,-2}-N_{2,3}^{2}+2 N_{2,3}=4 B_{-3,-2} B_{3,2}-N_{2,3}^{2}-2 N_{2,3} .
$$

By the same arguments as for (7.15a) we obtain

$$
-2 y_{-1} y_{1} \mathcal{L}_{\mathbb{C}^{2}}=-1+\mathcal{C}_{23}^{\mathrm{sch},-1}+\left(N_{1}^{\mathrm{sch},-1}\right)^{2} .
$$

Moreover, we have

$$
\left[B_{2,-1}, B_{-3,1}\right]=\left[B_{2,1}, B_{-3,-1}\right]=B_{-3,2} .
$$

The commutation relations (10.9) define two Heisenberg subalgebras

$$
\begin{aligned}
& \text { heis }_{+}\left(\mathbb{C}^{2}\right) \quad \text { spanned by } B_{2,-1}, B_{-3,1}, B_{-3,2} \text {, } \\
& \text { heis_ }\left(\mathbb{C}^{2}\right) \quad \text { spanned by } B_{2,1}, B_{-3,-1}, B_{-3,2} \text {. }
\end{aligned}
$$

Let us remark that heis $+\left(\mathbb{C}^{2}\right)$ is the $\tau_{1}$-image of heis $-\left(\mathbb{C}^{2}\right)$.

Let us define

$$
\begin{aligned}
& \mathcal{C}_{+}=2 B_{2,1} B_{-3,-1}+N_{2,3}+N_{1}-B_{-3,2}=2 B_{-3,-1} B_{2,1}+N_{2,3}+N_{1}+B_{-3,2}, \\
& \mathcal{C}_{-}=2 B_{2,-1} B_{-3,1}+N_{2,3}-N_{1}-B_{-3,2}=2 B_{-3,1} B_{2,-1}+N_{2,3}-N_{1}+B_{-3,2} .
\end{aligned}
$$

$\mathcal{C}_{+}$and $\mathcal{C}_{-}$can be viewed as the Casimir operators for heis $+\left(\mathbb{C}^{2}\right)$ and heis $-\left(\mathbb{C}^{2}\right)$ respectively. Indeed, $\mathcal{C}_{+}$, resp. $\mathcal{C}_{-}$commute with all operators in heis $\left(\mathbb{C}^{2}\right)$, resp. heis $\left(\mathbb{C}^{2}\right)$.

Let us now consider the operators on the level of $\mathbb{C}^{2} \oplus \mathbb{C}$. Direct calculation yields

$$
\mathcal{C}_{+}^{\text {sch }, \eta}=2 t\left(\partial_{y_{-1}} \partial_{y_{1}}+\partial_{t}\right)-\eta-1, \quad \mathcal{C}_{-}^{\text {sch }, \eta}=2 t\left(\partial_{y_{-1}} \partial_{y_{1}}+\partial_{t}\right)-\eta-1 .
$$

Therefore,

$$
t \mathcal{L}_{\mathbb{C}^{2}}=\mathcal{C}_{+}^{\mathrm{sch},-1}=\mathcal{C}_{-}^{\mathrm{sch},-1} .
$$

In the variables $w, u, s$ and after sandwiching with the exponential weight, (10.8) and (10.10) become

$$
-2 w s^{2} \hat{\mathcal{L}}_{\mathbb{C}^{2}}=-1+\hat{\mathcal{C}}_{23}^{\mathrm{sch},-1}+\left(\hat{N}_{1}^{\mathrm{sch},-1}\right)^{2}, \quad s^{2} \hat{\mathcal{L}}_{\mathbb{C}^{2}}=\hat{\mathcal{C}}_{+}^{\mathrm{sch},-1}=\hat{\mathcal{C}}_{-}^{\mathrm{sch},-1} .
$$

We apply the ansatz (10.6) and obtain all the factorizations of the balanced confluent operator of Section 2.3.

\section{$11 \operatorname{sch}\left(\mathbb{C}^{1}\right)$ and the Hermite equation}

In this section we derive the Hermite operator and its $\operatorname{sch}\left(\mathbb{C}^{1}\right)$ symmetries. We will consider the following levels:

(1) extended space $\mathbb{C}^{5}$ and the Laplacian,

(2) reduction to $\mathbb{C}^{3}$ and the Laplacian,

(3) reduction to $\mathbb{C} \oplus \mathbb{C}$ and the heat operator,

(4) special coordinates,

(5) sandwiching with a weight,

(6) separation of variables in (5) leads to the balanced Hermite operator,

(7) separation of variables in (4) leads to the standard Hermite operator. 


\section{$11.1 \quad \mathbb{C}^{5}$}

We again consider $\mathbb{C}^{5}$ with the coordinates (8.1) and the product given by the square (8.2). Remember that $\operatorname{sch}\left(\mathbb{C}^{1}\right)$ is a subalgebra of so $\left(\mathbb{C}^{5}\right)$ and we keep the notation from $\operatorname{so}\left(\mathbb{C}^{5}\right)$.

Lie algebra $\operatorname{sch}\left(\mathbb{C}^{1}\right)$. The Cartan algebra is spanned by

$$
N_{2,3}=z_{-2} \partial_{z_{-2}}-z_{2} \partial_{z_{2}}+z_{-3} \partial_{z_{-3}}-z_{3} \partial_{z_{3}}, \quad B_{-3,2}=z_{3} \partial_{z_{2}}-z_{-2} \partial_{z_{-3}} .
$$

Root operators:

$$
\begin{aligned}
& B_{2,0}=z_{-2} \partial_{z_{0}}-z_{0} \partial_{z_{2}}, \quad B_{-3,0}=z_{3} \partial_{z_{0}}-z_{0} \partial_{z_{-3}}, \quad B_{-3,-2}=z_{3} \partial_{z_{-2}}-z_{2} \partial_{z_{-3}}, \\
& B_{3,2}=z_{-3} \partial_{z_{2}}-z_{2} \partial_{z_{-3}} .
\end{aligned}
$$

\section{Weyl symmetry:}

$$
\kappa K\left(z_{0}, z_{-2}, z_{2}, z_{-3}, z_{3}\right)=K\left(z_{0},-z_{3},-z_{-3}, z_{2}, z_{-2}\right) .
$$

It generates a group isomorphic to $\mathbb{Z}_{4}$.

\section{$11.2 \mathbb{C}^{3}$}

We descend on the level of $\mathbb{C}^{3}$, as described in Section 8.6. In particular, we use the coordinates (8.12) with the scalar product given by (8.13).

Lie algebra $\operatorname{sch}\left(\mathbb{C}^{1}\right)$. Cartan algebra:

$$
N_{2,3}^{\mathrm{fl}, \eta}=y_{0} \partial_{y_{0}}+2 y_{-2} \partial_{y_{-2}}-\eta, \quad B_{-3,2}^{\mathrm{fl}}=\partial_{y_{2}} .
$$

Root operators:

$$
\begin{aligned}
& B_{2,0}^{\mathrm{fl}}=y_{-2} \partial_{y_{0}}-y_{0} \partial_{y_{2}}, \quad B_{-3,0}^{\mathrm{fl}}=\partial_{y_{0}}, \quad B_{-3,-2}^{\mathrm{fl}}=\partial_{y_{-2}}, \\
& B_{3,2}^{\mathrm{fl}, \eta}=y_{-2} y_{0} \partial_{y_{0}}+y_{-2}^{2} \partial_{y_{-2}}-\frac{1}{2} y_{0}^{2} \partial_{y_{2}}-\eta y_{2} .
\end{aligned}
$$

\section{Weyl symmetry:}

$$
\kappa^{\mathrm{fl}, \eta} f\left(y_{0}, y_{-2}, y_{2}\right)=y_{2}^{\eta} f\left(\frac{y_{0}}{y_{2}}, \frac{y_{0}^{2}+2 y_{-2} y_{2}}{2 y_{2}},-\frac{1}{y_{2}}\right) \text {. }
$$

\section{$11.3 \mathbb{C} \oplus \mathbb{C}$}

We descend onto the level of $\mathbb{C} \oplus \mathbb{C}$, as described in Section 9.4. $B_{-3,2}$ becomes equal to 1 , therefore it will be ignored further on. We rename $y_{-2}$ to $t$ and $y_{0}$ to $y$.

Lie algebra $\operatorname{sch}\left(\mathbb{C}^{1}\right)$. Cartan algebra:

$$
N_{2,3}^{\mathrm{sch}, \eta}=y \partial_{y}+2 t \partial_{t}-\eta
$$

Root operators:

$$
B_{2,0}^{\mathrm{sch}}=t \partial_{y}-y, \quad B_{-3,0}^{\mathrm{sch}}=\partial_{y}, \quad B_{-3,-2}^{\mathrm{sch}}=\partial_{t}, \quad B_{3,2}^{\mathrm{sch}, \eta}=t\left(y \partial_{y}+t \partial_{t}-\eta\right)-y^{2} .
$$

\section{Weyl symmetry:}

$$
\kappa^{\mathrm{sch}, \eta} h(y, t)=t^{\eta} \exp \left(\frac{y^{2}}{2 t}\right) h\left(\frac{y}{t},-\frac{1}{t}\right) \text {. }
$$




\section{Heat operator:}

$$
\mathcal{L}_{\mathbb{C}}=\partial_{y}^{2}+2 \partial_{t}
$$

\section{Generalized symmetries:}

$$
\begin{aligned}
& N_{2,3}^{\mathrm{sch},-\frac{5}{2}} \mathcal{L}_{\mathbb{C}}=\mathcal{L}_{\mathbb{C}} N_{2,3}^{\mathrm{sch},-\frac{1}{2}}, \\
& B_{i, j}^{\mathrm{sch},-\frac{5}{2}} \mathcal{L}_{\mathbb{C}}=\mathcal{L}_{\mathbb{C}} B_{i, j}^{\mathrm{sch},-\frac{1}{2}}, \quad(i, j)=(2,0),(-3,0), \pm(3,2), \\
& \kappa^{\mathrm{sch},-\frac{5}{2}} \mathcal{L}_{\mathbb{C}}=\mathcal{L}_{\mathbb{C}} \kappa^{\mathrm{sch},-\frac{1}{2}} .
\end{aligned}
$$

\subsection{Coordinates $w, s$}

Let us define new complex variables as

$$
w=\frac{y}{\sqrt{2 t}}, \quad s=\sqrt{t} .
$$

Reverse transformations are

$$
y=\sqrt{2} s w, \quad t=s^{2} .
$$

Lie algebra $\operatorname{sch}\left(\mathbb{C}^{1}\right)$. Cartan operators:

$$
N_{2,3}^{\mathrm{sch}, \eta}=s \partial_{s}-\eta
$$

Root operators:

$$
\begin{aligned}
& B_{-3,0}^{\mathrm{sch}}=\frac{1}{\sqrt{2} s} \partial_{w}, \quad B_{2,0}^{\mathrm{sch}}=\frac{s}{\sqrt{2}}\left(\partial_{w}-2 w\right), \\
& B_{-3,-2}^{\mathrm{sch}}=\frac{1}{2 s^{2}}\left(-w \partial_{w}+N_{2,3}^{\mathrm{sph}, \eta}+\eta\right), \quad B_{3,2}^{\mathrm{sch}, \eta}=\frac{s^{2}}{2}\left(w \partial_{w}+N_{2,3}^{\mathrm{sph}, \eta}-\eta-2 w^{2}\right) .
\end{aligned}
$$

Above $B_{-3,-2}^{\text {sch }}$ does not depend on $\eta$ even if at first glance it might seem so.

\section{Weyl symmetry:}

$$
\kappa^{\mathrm{sch}, \eta} h(w, s)=s^{2 \eta} \mathrm{e}^{w^{2}} h\left(\mathrm{i} w,-\frac{\mathrm{i}}{s}\right) .
$$

\section{Heat operator:}

$$
\mathcal{L}_{\mathbb{C}}=\frac{1}{2 s^{2}}\left(\partial_{w}^{2}-2 w \partial_{w}+2 s \partial_{s}\right)
$$

\subsection{Sandwiching with a Gaussian}

For any operator $C$ we will write

$$
\hat{C}:=\mathrm{e}^{-\frac{w^{2}}{2}} C \mathrm{e}^{\frac{w^{2}}{2}} .
$$

Lie algebra $\operatorname{sch}\left(\mathbb{C}^{1}\right)$. Cartan algebra:

$$
\hat{N}_{2,3}^{\mathrm{sch}, \eta}=s \partial_{s}-\eta .
$$


Root operators:

$$
\begin{aligned}
& \hat{B}_{-3,0}^{\mathrm{sch}}=\frac{1}{\sqrt{2} s}\left(\partial_{w}+w\right), \quad \hat{B}_{2,0}^{\mathrm{sch}}=\frac{s}{\sqrt{2}}\left(\partial_{w}-w\right), \\
& \hat{B}_{-3,-2}^{\mathrm{sch}}=\frac{1}{2 s^{2}}\left(-w \partial_{w}+N_{23}^{\mathrm{sph}, \eta}+\eta-w^{2}\right), \quad \hat{B}_{3,2}^{\mathrm{sch}, \eta}=\frac{s^{2}}{2}\left(w \partial_{w}+N_{23}^{\mathrm{sph}, \eta}-\eta-w^{2}\right) .
\end{aligned}
$$

\section{Weyl symmetry:}

$$
\kappa^{\mathrm{sch}, \eta} h(w, s)=s^{2 \eta} h\left(\mathrm{i} w,-\frac{\mathrm{i}}{s}\right) .
$$

\section{Heat operator:}

$$
\hat{\mathcal{L}}_{\mathbb{C}}=\frac{1}{2 s^{2}}\left(\partial_{w}^{2}-w^{2}-2 N_{2,3}^{\mathrm{sch},-\frac{1}{2}}\right) .
$$

\subsection{Balanced Hermite operator}

We make an ansatz

$$
h(w, s)=s^{\lambda-\frac{1}{2}} F(w) .
$$

Clearly,

$$
\hat{N}_{2,3}^{\mathrm{sch},-\frac{1}{2}} h=\lambda h .
$$

Therefore, on functions of this form, $2 s^{2} \hat{\mathcal{L}}_{\mathbb{C}^{1}}$ coincides with the balanced Hermite operator (2.8). The generalized symmetries for the roots (11.2) and for the Weyl symmetry (11.3) coincide with the transmutation relations and the discrete symmetry of the balanced Hermite operator, respectively; see Section 2.4.

\subsection{Standard Hermite operator}

Alternatively, we can use the ansatz

$$
h(w, s)=s^{\lambda-\frac{1}{2}} F(w) .
$$

without the sandwiching (11.4). Clearly,

$$
N_{2,3}^{\mathrm{sch},-\frac{1}{2}} h=\lambda h .
$$

Then, on functions of the form $(11.6), 2 s^{2} \mathcal{L}_{\mathbb{C}^{2}}$ coincides with the standard Hermite operator (2.7).

\subsection{Factorizations}

In $\operatorname{sch}\left(\mathbb{C}^{1}\right)$ we have a distinguished subalgebra isomorphic to $\operatorname{so}\left(\mathbb{C}^{3}\right)$

$$
\mathrm{SO}_{23}\left(\mathbb{C}^{3}\right) \quad \text { spanned by } B_{-3,-2}, B_{3,2}, N_{2,3},
$$

and a distinguished Heisenberg algebra

$$
\text { heis }_{0}\left(\mathbb{C}^{2}\right) \quad \text { spanned by } B_{2,0}, B_{-3,0}, B_{-3,2} \text {. }
$$

We set

$$
\begin{aligned}
& \mathcal{C}_{23}=4 B_{3,2} B_{-3,-2}-N_{2,3}^{2}+2 N_{2,3}=4 B_{-3,-2} B_{3,2}-N_{2,3}^{2}-2 N_{2,3}, \\
& \mathcal{C}_{0}=2 B_{2,0} B_{-3,0}+2 N_{2,3}-B_{-3,2}=2 B_{-3,0} B_{2,0}+2 N_{2,3}+B_{-3,2} .
\end{aligned}
$$


$\mathcal{C}_{23}$ is the Casimir operator of $\mathrm{so}_{23}\left(\mathbb{C}^{3}\right) . \mathcal{C}_{0}$ can be treated as the Casimir operator of heis $0\left(\mathbb{C}^{2}\right)$. We have the identities

$$
-y_{0}^{2} \mathcal{L}_{\mathbb{C}}=\mathcal{C}_{23}^{\mathrm{sch},-\frac{1}{2}}-\frac{3}{4}, \quad 2 t \mathcal{L}_{\mathbb{C}}=\mathcal{C}_{0}^{\mathrm{sch},-\frac{1}{2}} .
$$

In the coordinates $w, s$ we can rewrite this as

$$
-w^{2} 2 s^{2} \hat{\mathcal{L}}_{\mathbb{C}}=\hat{\mathcal{C}}_{23}^{\mathrm{sch},-\frac{1}{2}}-\frac{3}{4}, \quad 2 s^{2} \hat{\mathcal{L}}_{\mathbb{C}}=\hat{\mathcal{C}}_{0}^{\mathrm{sch},-\frac{1}{2}}
$$

We apply the ansatz (11.5) and obtain all the factorizations of the balanced Hermite operator of Section 2.4.

\section{$12 \mathbb{C}^{2} \rtimes \operatorname{so}\left(\mathbb{C}^{2}\right)$ and the ${ }_{0} F_{1}$ equation}

In this section we derive the ${ }_{0} F_{1}$ operator and its $\mathbb{C}^{2} \rtimes \mathrm{so}\left(\mathbb{C}^{2}\right)$ symmetries from the symmetries of the Helmholtz equation in 2 dimensions. One can argue that this is the simplest case among the five cases considered in this paper, because only true (that is, not generalized) symmetries are used here. This derivation is also extensively discussed in the literature. (Strictly speaking, in the literature usually the Bessel and modified Bessel equations are considered. They are, however, equivalent to the ${ }_{0} F_{1}$ equation, as described, e.g., in [6].) We included this section for the sake of completeness.

Perhaps, it would be sufficient to discuss only two levels of derivation - the 2-dimensional Helmholtz equation and the ${ }_{0} F_{1}$ equation. However, to make this section easier to compare with the previous ones, we will start from a higher level.

Thus, we will consider the following levels:

(1) space $\mathbb{C}^{5}$ and the Laplacian $\Delta_{\mathbb{C}^{5}}$,

(2) space $\mathbb{C}^{3}$ and the Laplacian $\Delta_{\mathbb{C}^{3}}$,

(3) space $\mathbb{C}^{2}$ and the Helmholtz operator $\Delta_{\mathbb{C}^{2}}-1$,

(4) choosing appropriate coordinates we obtain the ${ }_{0} F_{1}$ operator.

\subsection{Space $\mathbb{C}^{5}$}

As in Section 8.1, we consider $\mathbb{C}^{5}$ with the coordinates

$$
z_{0}, z_{-2}, z_{2}, z_{-3}, z_{3}
$$

and the product given by

$$
\langle z \mid z\rangle=z_{0}^{2}+2 z_{-2} z_{2}+2 z_{-3} z_{3}
$$

Lie algebra $\mathbb{C}^{2} \rtimes \mathrm{so}\left(\mathbb{C}^{2}\right)$ on $\mathbb{C}^{5}$. Cartan operator:

$$
N_{2}=z_{-2} \partial_{z_{-2}}-z_{2} \partial_{z_{2}} .
$$

Root operators:

$$
B_{-3,-2}=z_{3} \partial_{-2}-z_{2} \partial_{-3}, \quad B_{-3,2}=z_{3} \partial_{2}-z_{-2} \partial_{-3} .
$$

Weyl symmetry. Flip:

$$
\tau_{2} K\left(z_{0}, z_{-2}, z_{2}, z_{-3}, z_{3}\right)=K\left(z_{0}, z_{2}, z_{-2}, z_{-3}, z_{3}\right) .
$$

\section{Laplacian:}

$$
\Delta_{\mathbb{C}^{5}}=\partial_{z_{0}}^{2}+2 \partial_{z_{-2}} \partial_{z_{2}}+2 \partial_{z_{-3}} \partial_{z_{3}} .
$$




\subsection{Space $\mathbb{C}^{3}$}

As in Section 8.6, we consider $\mathbb{C}^{3}$ with coordinates $\left(y_{0}, y_{-2}, y_{2}\right)$ and the scalar product given by

$$
\langle y \mid y\rangle=y_{0}^{2}+2 y_{-2} y_{2} .
$$

Lie algebra $\mathbb{C}^{2} \rtimes \operatorname{so}\left(\mathbb{C}^{2}\right)$. Cartan operator:

$$
N_{2}^{\mathrm{fl}}=y_{-2} \partial_{y_{-2}}-y_{2} \partial_{y_{2}} .
$$

\section{Root operators:}

$$
B_{-3,-2}^{\mathrm{fl}}=\partial_{y_{-2}}, \quad B_{-3,2}^{\mathrm{fl}}=\partial_{y_{2}} .
$$

Weyl symmetry. Flip:

$$
\tau_{2}^{\mathrm{fl}} f\left(y_{0}, y_{-2}, y_{2}\right)=f\left(y_{0}, y_{2}, y_{-2}\right)
$$

\section{Reduced Laplacian:}

$$
\Delta_{\mathbb{C}^{5}}^{\mathrm{fl}}=\Delta_{\mathbb{C}^{3}}=\partial_{y_{0}}^{2}+2 \partial_{y_{-2}} \partial_{y_{2}} .
$$

\subsection{Space $\mathbb{C}^{2}$ and the Helmholtz equation}

We make an asatz

$$
f\left(y_{0}, y_{-}, y_{+}\right)=\mathrm{e}^{-y_{0}} h\left(y_{-}, y_{+}\right) .
$$

In particular, the coordinates $y_{-2}, y_{2}$ are renamed to $y_{-}, y_{+}$. We also simplify the names of various operators in an obvious way.

Lie algebra $\mathbb{C}^{2} \rtimes \operatorname{so}\left(\mathbb{C}^{2}\right)$. Cartan operator:

$$
N=y_{-} \partial_{y_{-}}-y_{+} \partial_{y_{+}}
$$

Root operators:

$$
B_{-}=\partial_{y_{-}}, \quad B_{+}=\partial_{y_{+}}
$$

Weyl symmetry. Flip

$$
\tau f\left(y_{-}, y_{+}\right)=f\left(y_{+}, y_{-}\right)
$$

\section{Helmholtz operator:}

$$
\mathcal{K}_{\mathbb{C}^{2}}:=-1+2 \partial_{y_{-}} \partial_{y_{+}}
$$

\section{Symmetries:}

$$
N \mathcal{K}_{\mathbb{C}^{2}}=\mathcal{K}_{\mathbb{C}^{2}} N, \quad B_{ \pm} \mathcal{K}_{\mathbb{C}^{2}}=\mathcal{K}_{\mathbb{C}^{2}} B_{ \pm}, \quad \tau \mathcal{K}_{\mathbb{C}^{2}}=\mathcal{K}_{\mathbb{C}^{2}} \tau
$$




\subsection{Balanced ${ }_{0} F_{1}$ operator}

We introduce the coordinates

$$
w=\frac{y_{-} y_{+}}{2}, \quad u=\sqrt{\frac{y_{-}}{y_{+}}} .
$$

Lie algebra $\mathbb{C}^{2} \rtimes \mathrm{so}\left(\mathbb{C}^{2}\right)$. Cartan operator:

$$
N=u \partial_{u} .
$$

Root operators:

$$
B_{+}=u \frac{1}{\sqrt{2 w}}\left(w \partial_{w}-\frac{N_{1}}{2}\right), \quad B_{-}=\frac{1}{u} \frac{1}{\sqrt{2 w}}\left(w \partial_{w}+\frac{N_{1}}{2}\right) .
$$

Weyl symmetry. Flip:

$$
\tau h(w, u)=h\left(w, \frac{1}{u}\right) .
$$

\section{Helmholtz operator:}

$$
\mathcal{K}_{\mathbb{C}^{2}}=\partial_{w} w \partial_{w}-\frac{N^{2}}{4 w}-1 .
$$

Making an ansatz

$$
h(w, u)=u^{\alpha} F(w),
$$

we obtain the balanced ${ }_{0} F_{1}$ operator. Symmetries for the root operators and the flip coincide with the transmutation relation and the change of the sign of $\alpha$ in the balanced ${ }_{0} F_{1}$ operator, respectively; see Section 2.5.

\subsection{Standard ${ }_{0} F_{1}$ operator}

Modify the coordinates (12.1) by replacing $u$ with

$$
\tilde{u}:=y_{-}=u \sqrt{2 w} \text {. }
$$

We then have

$$
N=\tilde{u} \partial_{\tilde{u}}, \quad \mathcal{K}_{\mathbb{C}^{2}}=w \partial_{w}^{2}+(1+N) \partial_{w}-1 .
$$

Making an ansatz

$$
h(w, u)=\tilde{u}^{\alpha} \tilde{F}(w),
$$

we obtain the standard ${ }_{0} F_{1}$ operator.

\subsection{Factorizations}

The factorizations

$$
\mathcal{K}_{\mathbb{C}^{2}}=2 B_{-} B_{+}-1=2 B_{+} B_{-}-1
$$

are completely obvious. They yield the factorizations of the ${ }_{0} F_{1}$ operator. 


\section{Acknowledgements}

We thank Tom Koornwinder and anonymous referees for useful remarks. J.D. gratefully acknowledges financial support of the National Science Center, Poland, under the grant UMO2014/15/B/ST1/00126.

\section{References}

[1] Andrews G.E., Askey R., Roy R., Special functions, Encyclopedia of Mathematics and its Applications, Vol. 71, Cambridge University Press, Cambridge, 1999.

[2] Beukers F., Hypergeometric functions, how special are they?, Notices Amer. Math. Soc. 61 (2014), 48-56.

[3] Bôcher M., Über die Reihenentwickelungen Potentialtheorie, Teubner, Leipzig, 1894.

[4] Bod E., Algebraic $\mathcal{A}$-hypergeometric functions and their monodromy, Ph.D. Thesis, Utrecht University, 2013.

[5] Cooper F., Khare A., Sukhatme U., Supersymmetry in quantum mechanics, World Sci. Publ. Co., Inc., River Edge, NJ, 2001.

[6] Dereziński J., Hypergeometric type functions and their symmetries, Ann. Henri Poincaré 15 (2014), 15691653, arXiv:1305.3113.

[7] Dereziński J., Wrochna M., Exactly solvable Schrödinger operators, Ann. Henri Poincaré 12 (2011), 397418, arXiv:1009.0541.

[8] Erdélyi A., Magnus W., Oberhettinger F., Tricomi F.G., Higher transcendental functions. Vol. I, McGrawHill Book Company, New York, 1953.

[9] Erdélyi A., Magnus W., Oberhettinger F., Tricomi F.G., Higher transcendental functions. Vol. II, McGrawHill Book Company, New York, New York, 1953.

[10] Erdélyi A., Magnus W., Oberhettinger F., Tricomi F.G., Higher transcendental functions. Vol. III, McGrawHill Book Company, New York, New York, 1955.

[11] Flügge S., Practical quantum mechanics, Classics in Mathematics, Vol. 177, Springer-Verlag, Berlin, 1999.

[12] Hagen C.R., Scale and conformal transformations in galilean-covariant field theory, Phys. Rev. D 5 (1972), $377-388$.

[13] Hochstadt H., The functions of mathematical physics, Pure and Applied Mathematics, Vol. 23, WileyInterscience, New York - London - Sydney, 1971.

[14] Infeld L., Hull T.E., The factorization method, Rev. Modern Phys. 23 (1951), 21-68.

[15] Kalnins E.G., Miller Jr. W., Lie theory and the wave equation in space-time. IV. The Klein-Gordon equation and the Poincaré group, J. Math. Phys. 19 (1978), 1233-1246.

[16] Kalnins E.G., Miller Jr. W., The wave equation and separation of variables on the complex sphere $S_{4}$, J. Math. Anal. Appl. 83 (1981), 449-469.

[17] Kalnins E.G., Miller Jr. W., Reid G.J., Separation of variables for complex Riemannian spaces of constant curvature. I. Orthogonal separable coordinates for $\mathrm{S}_{n} \mathbf{C}$ and $\mathrm{E}_{n \mathbf{C}}$, Proc. Roy. Soc. London Ser. A 394 (1984), $183-206$.

[18] Kobayashi S., Nomizu K., Foundations of differential geometry, Vol. I, John Wiley \& Sons, Inc., New York London - Sydney, 1963.

[19] Kobayashi S., Nomizu K., Foundations of differential geometry. Vol. II, John Wiley \& Sons, Inc., New York - London - Sydney, 1969.

[20] Koornwinder T.H., Fractional integral and generalized Stieltjes transforms for hypergeometric functions as transmutation operators, SIGMA 11 (2015), 074, 22 pages, arXiv:1504.08144.

[21] Kummer E.E., Über die hypergeometrische Reihe $1+\frac{\alpha \cdot \beta}{1 \cdot \gamma} x+\frac{\alpha(\alpha+1) \beta(\beta+1)}{1 \cdot 2 \cdot \gamma(\gamma+1)} x^{2}+\frac{\alpha(\alpha+1)(\alpha+2) \beta(\beta+1)(\beta+2)}{1 \cdot 2 \cdot 3 \cdot \gamma(\gamma+1)(\gamma+2} x^{3}+\cdots$, J. Reine Angew. Math. 15 (1836), 39-83.

[22] Kummer E.E., Über die hypergeometrische Reihe $1+\frac{\alpha \cdot \beta}{1 \cdot \gamma} x+\frac{\alpha(\alpha+1) \beta(\beta+1)}{1 \cdot 2 \cdot \gamma(\gamma+1)} x^{2}+\frac{\alpha(\alpha+1)(\alpha+2) \beta(\beta+1)(\beta+2)}{1 \cdot 2 \cdot 3 \cdot \gamma(\gamma+1)(\gamma+2} x^{3}+\cdots$, J. Reine Angew. Math. 15 (1836), 127-172.

[23] Kuznetsov V., PG course on special functions and their symmetries, 2003, available at http://www1.maths . leeds.ac.uk/ kisilv/courses/sp-funct.pdf. 
[24] Lie S., Ueber die Integration durch bestimmte Integration von einer Classe linearer partieller Differentialgleichungen, Arch. Math. Naturvid. 6 (1881), 328-368.

[25] Lievens S., Rao K.S., Van der Jeugt J., The finite group of the Kummer solutions, Integral Transforms Spec. Funct. 16 (2005), 153-158.

[26] Magnus W., Oberhettinger F., Soni R.P., Formulas and theorems for the special functions of mathematical physics, Die Grundlehren der mathematischen Wissenschaften, Vol. 52, 3rd ed., Springer-Verlag, New York, 1966.

[27] Miller Jr. W., Lie theory and special functions, Mathematics in Science and Engineering, Vol. 43, Academic Press, New York - London, 1968.

[28] Miller Jr. W., Symmetry groups and their applications, Pure and Applied Mathematics, Vol. 50, Academic Press, New York - London, 1972.

[29] Miller Jr. W., Lie theory and generalizations of the hypergeometric functions, SIAM J. Appl. Math. 25 (1973), 226-235.

[30] Miller Jr. W., Symmetries of differential equations. The hypergeometric and Euler-Darboux equations, SIAM J. Math. Anal. 4 (1973), 314-328.

[31] Miller Jr. W., Symmetry and separation of variables, Encyclopedia of Mathematics and its Applications, Vol. 4, Addison-Wesley Publishing Co., Reading, Mass. - London - Amsterdam, 1977.

[32] Morse P.M., Feshbach H., Methods of theoretical physics, Vols. I, II, McGraw-Hill Book Co., Inc., New York - Toronto - London, 1953.

[33] Niederer U., The maximal kinematical invariance group of the free Schrödinger equation, Helv. Phys. Acta 45 (1972), 802-810.

[34] Nikiforov A.F., Uvarov V.B., Special functions of mathematical physics. A unified introduction with applications, Birkhäuser Verlag, Basel, 1988.

[35] Olver F.W.J., Lozier D.W., Boisvert R.F., Clark C.W. (Editors), NIST handbook of mathematical functions, U.S. Department of Commerce, National Institute of Standards and Technology, Washington, DC, Cambridge University Press, Cambridge, 2010, available at http://dlmf.nist.gov/.

[36] Olver P.J., Applications of Lie groups to differential equations, Graduate Texts in Mathematics, Vol. 107, Springer-Verlag, New York, 1986.

[37] Ørsted B., Conformally invariant differential equations and projective geometry, J. Funct. Anal. 44 (1981), $1-23$.

[38] Rainville E.D., Special functions, The Macmillan Co., New York, 1960.

[39] Saito M., Symmetry algebras of normal $\mathcal{A}$-hypergeometric systems, Hokkaido Math. J. 25 (1996), 591-619.

[40] Schrödinger E., A method of determining quantum-mechanical eigenvalues and eigenfunctions, Proc. Roy. Irish Acad. Sect. A. 46 (1940), 9-16.

[41] Taylor M.E., Noncommutative harmonic analysis, Mathematical Surveys and Monographs, Vol. 22, Amer. Math. Soc., Providence, RI, 1986.

[42] Truesdell C., An essay toward a unified theory of special functions based upon the functional equation $(\partial / \partial z) F(z, \alpha)=F(z, \alpha+1)$, Annals of Mathematics Studies, Vol. 18, Princeton University Press, Princeton, NJ, 1948.

[43] Vilenkin N.Ja., Special functions and the theory of group representations, Translations of Mathematical Monographs, Vol. 22, Amer. Math. Soc., Providence, RI, 1968.

[44] Vilenkin N.Ja., Klimyk A.U., Representation of Lie groups and special functions, Vol. 1, Simplest Lie groups, special functions and integral transforms, Mathematics and its Applications (Soviet Series), Vol. 72, Kluwer Academic Publishers Group, Dordrecht, 1991.

[45] Wawrzyńczyk A., Współczesna teoria funkcji specjalnych, Biblioteka Matematyczna, Vol. 52, Państwowe Wydawnictwo Naukowe (PWN), Warsaw, 1978.

[46] Weisner L., Generating functions for Hermite functions, Canad. J. Math. 11 (1959), 141-147.

[47] Weisner L., Generating functions for Bessel functions, Canad. J. Math. 11 (1959), 148-155.

[48] Whittaker E.T., Watson G.N., A course of modern analysis, Cambridge Mathematical Library, Cambridge University Press, Cambridge, 1996. 